Portland State University

PDXScholar

3-3-2020

\title{
Minimum Complexity Echo State Networks for Genome and Sequence Analysis
}

Christopher John Neighbor

Portland State University

Follow this and additional works at: https://pdxscholar.library.pdx.edu/open_access_etds

Part of the Bioinformatics Commons

Let us know how access to this document benefits you.

\section{Recommended Citation}

Neighbor, Christopher John, "Minimum Complexity Echo State Networks for Genome and Sequence Analysis" (2020). Dissertations and Theses. Paper 5502.

https://doi.org/10.15760/etd.7376

This Thesis is brought to you for free and open access. It has been accepted for inclusion in Dissertations and Theses by an authorized administrator of PDXScholar. Please contact us if we can make this document more accessible: pdxscholar@pdx.edu. 
Minimum Complexity Echo State Networks for Genome and Sequence Analysis

by

Christopher John Neighbor

A thesis submitted in partial fulfillment of the requirements for the degree of

\author{
Master of Science \\ in \\ Electrical and Computer Engineering
}

Thesis Committee:

Christof Teuscher, Chair

John Lipor

Ted Willke

Portland State University

2020 


\begin{abstract}
Increasing viral illnesses threatens global human health and welfare. Due to the distribution of disease and the expense of diagnosis, it is of value to develop portable assays that can detect viral infections early. DNA molecular logic technology offers a portable detection method due to the versatility and stability of DNA and the potential of in situ computation.

Top-down engineering of these chemical logic networks can be difficult due to the difficulties of their implementation using DNA as a substrate. In this work echo state networks, a form of recurrent neural networks, were explored with the motivation that their implementation in situ would be more feasible. Echo state networks consist of a fixed recurrent hidden layer, called the reservoir, with a trainable linear readout layer. We explored the size and sparsity of these networks with an aim for minimum complexity, small size and fewer connections, while still achieving improvements over linear baseline models.

The performance of these networks was compared against the NARMA benchmark time series prediction task and the classification tasks of digit recognition, gene splice junctions, and Dengue virus genome serotypes. The networks were able to perform the tasks for NARMA achieving improvements over memoryless linear models with only 2 nodes and over linear models with input memory with 100 nodes. For the digit recognition task, a 20 node and 10\% connectivity network improved performance over linear models. For the genome sequence classification tasks these networks could achieve $>90 \%$ accuracy on each providing improvements from linear models of $2.8 \%$ for the splice junctions and $13.6 \%$ for Dengue virus classification.

To bridge the model of echo state networks to chemical reaction networks, an oscillating recurrent neural network was simulated which was based on the graph
\end{abstract}


of an oscillating chemical reaction network. Performance was characterized across size and spectral radius of the network. For the NARMA task, an improvement in mean squared error of near an order of magnitude could be achieved by increasing the oscillator from having 6 nodes to having 300 nodes and a similar improvement in performance between a spectral radius of 0.1 and 0.9 was observed. This was also compared to a simulated chemical oscillator in terms of prediction accuracy and classification on the previous tasks.

The simulated results from the classifier suggest that a chemical reaction network of somewhat unknown connectivity and limited complexity but sufficient dynamics could be a potentially easier means of implementing an in situ viral classifier than the traditional methods of top-down engineering. This method will be transferable to other pathogens, both viral and bacterial, using available genome databases. 


\section{Acknowledgements}

Firstly, I would like to thank my thesis advisor, Dr. Christof Teuscher, for his support and guidance during this tremendous learning experience. His passion and enthusiasm for teaching and learning were contagious and provided a positive work environment in which to grow.

Thank you to my committee members, Dr. John Lipor and Dr. Ted Willke. Their classes taught me things I was able to apply to my thesis research. Their questions made me think deeper about the "why" of my results and helped me to understand my work better and to be able to convey my results in a clear, concise manner.

Thank you to Dr. Adán Myers y Gutiérrez for his advice on ways to implement these systems biochemically. If I asked him a question he couldn't answer he would go to his advisors and come back with an informative response.

Much appreciation goes to Dr. Darko Stefanovic for his encouragement and advice at our group meetings.

I am grateful for the funding of this research which is based upon work supported by the National Science Foundation under grant no. 1518833.

Finally, I must express my very profound gratitude to my wife, Caity, for her

long-suffering and unfailing support and encouragement for getting me through the experiment which was me writing this thesis. This accomplishment would not have been possible without them. Thank you. 


\section{Contents}

Abstract $\quad$ i

Acknowledgements $\quad$ iii

List of Tables $\quad$ vii

List of Figures $\quad$ viii

1 Introduction 1

1.1 Goals and Motivations . . . . . . . . . . . . . . . . 1

1.2 Challenges . . . . . . . . . . . . . . . . . 4

1.3 Contributions ......................... 4

$\begin{array}{lll}2 & \text { Background } & 7\end{array}$

2.1 Neural Networks . . . . . . . . . . . . . . . . . . 7

2.1.1 Feed Forward Neural Networks . . . . . . . . . . . . . 7

2.1.1.1 Gradient Descent . . . . . . . . . . . . . 9

2.1.1.2 Activation Functions . . . . . . . . . . . . 9

2.1.2 Convolutional Neural Networks . . . . . . . . . . . . . . . . . 10

2.1.3 Recurrent Neural Networks . . . . . . . . . . . . . . . . . . . 11

2.2 Reservoir Computing . . . . . . . . . . . . . . . . . . 12

2.2.1 Readout Layer Training . . . . . . . . . . . . . . 16

2.2.1.1 Linear Regression . . . . . . . . . . . . 17

2.2.1.2 Ridge Regression . . . . . . . . . . . . . . 18

2.2.1.3 Classification Readout Layer . . . . . . . . . . . . . 19 
2.2.1.4 Error Measures . . . . . . . . . . . . . . 20

2.3 Chemical Reaction Networks . . . . . . . . . . . . . . . . . 22

2.3.1 DNA Strand Displacement . . . . . . . . . . . . . . 23

2.3.2 Oscillating Chemical Networks . . . . . . . . . . . . . 24

2.3.3 Gillespie Chemical Simulation . . . . . . . . . . . . . . 27

2.3.4 K-mer Extraction . . . . . . . . . . . . . . . . . 28

2.4 Related Work . . . . . . . . . . . . . . . . . . 29

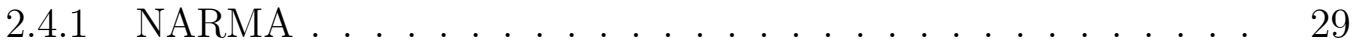

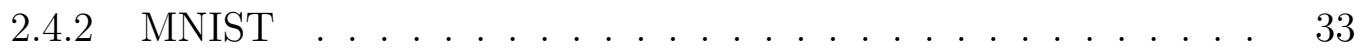

2.4.3 Splice Junction Gene Sequences . . . . . . . . . . . . . . . . 35

2.4.4 Dengue Virus Genome . . . . . . . . . . . . . . 36

2.4.5 Other Dataset Explorations . . . . . . . . . . . . 38

3 Design Methodology 40

3.1 Implementation of the Echo State Network Simulations and Readout Layer . . . . . . . . . . . . . . . . . . . . . 41

3.1.1 Parameter Selection . . . . . . . . . . . . . . 42

3.1.2 Oscillating Neural Network . . . . . . . . . . . . . . . . 47

3.1.3 Chemical Oscillator as a Reservoir . . . . . . . . . . . . 50

3.1.4 Encoding DNA Sequences . . . . . . . . . . . . 51

3.1.5 Training and Validation ............. 53

3.2 Physical Implementation . . . . . . . . . . . . . . . . 54

3.2.1 DV Serotype Detection Assay . . . . . . . . . . . . . 54

3.2.2 Determining Biochemical Constraints of the System . . . . . . 55

$\begin{array}{llr}4 & \text { Results } & 57\end{array}$

4.1 Sequence Prediction . . . . . . . . . . . . . . . 57 
4.1.1 NARMA Task A . . . . . . . . . . . . . . . . . 58

4.1.1.1 Echo State Network . . . . . . . . . . . . 58

4.1.1.2 Oscillator Networks . . . . . . . . . . . . . 60

$4.1 .2 \quad$ NARMA-10 . . . . . . . . . . . . . . . 61

4.1.2.1 Echo State Networks . . . . . . . . . . . . . 61

4.1.2.2 Oscillator Networks . . . . . . . . . . . . 63

4.2 Sequence Classification . . . . . . . . . . . . . . . 65

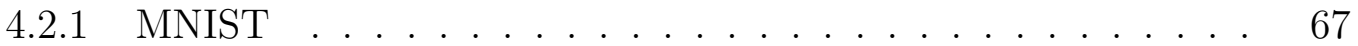

4.2.1.1 Echo State Networks . . . . . . . . . . . . . . . 67

4.2.1.2 Oscillator Networks . . . . . . . . . . 70

4.2 .2 Splice Junction Gene Sequences . . . . . . . . . . . . . . . . 72

4.2.2.1 Echo State Networks . . . . . . . . . . . . . 72

4.2.2.2 Oscillator Networks . . . . . . . . . . . . . . . 74

4.2.3 Dengue Virus Genome . . . . . . . . . . . . . . . . 76

4.2.3.1 Echo State Networks . . . . . . . . . . . . 76

4.2.3.2 Oscillator Networks . . . . . . . . . . . . . . 79

5 Conclusion $\quad 82$

$\begin{array}{lr}\text { References } & 86\end{array}$

$\begin{array}{ll}\text { Appendix: Tables of Results } & 97\end{array}$ 


\section{List of Tables}

4.1 Performances of the ESN, oscillating neural network and the chemical oscillator for the NARMA tasks. $\mathrm{sp}=$ sparsity, $\mathrm{SR}=$ spectral radius, the errors are given in MSE. The minimum complexity is when the ESN significantly surpasses the memoryless linear models and linear models with input memory which was represented in the result figures by the blue and red lines. . . . . . . . . . . . . . . . . . 66

4.2 Classification accuracy of the ESN, oscillating neural network and the chemical oscillator for the classification tasks. $\mathrm{sp}=$ sparsity, $\mathrm{SR}=$ spectral radius. The minimum complexity is when the ESN significantly surpasses the logistic regression model represented in the results figures by the blue lines. . . . . . . . . . . . . . . . . . 


\section{List of Figures}

1.1 Showing some machine learning methods and previous work which has mapped these methods of machine learning onto a chemical substrate. The names on the arrows are references to previous research which has been done to bridge the gap between the mathematical machine learning methods and their implementation in chemistry Hjelmfelt [1], Lopez [2], Cherry [3], Qian [4]. The aim of this research is to explore some methods of bridging the gap between the machine learning methods in Reservoir Computing such as oscillating networks, Dion [5], and echo state networks, Jaeger [6], and their chemical implementation such as by using chemical oscillators, Goudarzi [7]. This could result in an improved scaling up of these networks to handle more complex tasks. Chemical reaction networks can be constructed using DNA strands and methods described $[8] \ldots \ldots \ldots$

2.1 The perceptron model which takes the weighted summation of the inputs and then passes them through an activation function to generate

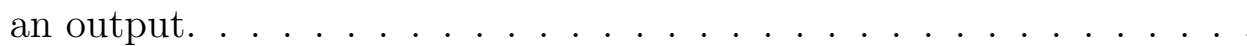

2.2 A multi-layered perceptron network, the input is fed into the input layer and passed forward through the hidden layer then on to the output layer, each of the nodes within the hidden layer are a perceptron. Due to its multi-layered structure it is capable of solving non-linear

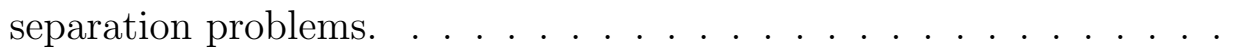


2.3 The common non-linear activation functions for neural networks of sigmoid, tanh, and ReLU. . . . . . . . . . . . . . .

2.4 Simple RNN node with the recurrent connection in the hidden layer. Note the output node can be connected to the hidden layer node's output across time thus adding to the time dependencies. A typical activation function for the recurrent node is $\tanh . \ldots . . . .$.

2.5 Layout of a Reservoir. The input is connected with the randomly initialized weight matrix, $W_{i n}$, into the reservoir whose fixed connection matrix, $W_{\text {res }}$, has been randomly initialized and fixed, the output states of the reservoir layer are then passed to a linear readout layer whose connection matrix, $W_{\text {out }}$, can be updated and trained for the particular

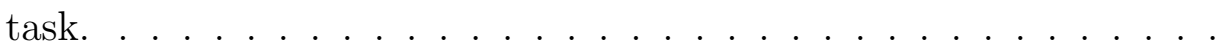

2.6 Chemical oscillations present in the Lotka-Volterra (Predator-Prey) reactions. Present in this system is also a chemical fuel source which is used to produce the Prey chemical, however, it is present at a large enough quantity to be held constant. . . . . . . . . . . . .

2.7 Oscillatory behavior from the displacillator chemical reaction equations which have been implemented using DNA strand circuits [9]. These reactions are explored as a reservoir layer where the input into the system is a scaled concentration of chemical $A$ and a linear readout layer of the chemical values is used. . . . . . . . . . . .

2.8 Example of how the feature preprocessing step of k-mer extraction works. A sliding window of size $k$ is moved along the genome and the count of each of short sequences of length $k$ is tracked giving a k-mer frequency distribution. . . . . . . . . . . . . . . . . 
2.9 Example of the NARMA-10 sequence generated from the random vector input. From the equation it can be seen that the output is constructed from a scaled previous output, the previous input times the scaled moving average of the previous 10 outputs, a combination of previous inputs 10 steps apart, and add a constant. . . . . . . . . .

2.10 NARMA-10 autocorrelation plot showing the time correlations of the sequence, shaded blue area is $95 \%$ confidence intervals of correlation.

2.11 Diagram of an example MNIST digit which is $28 \times 28$ pixels. The columns are treated as time steps and the rows are used as input channels, thus converting the task into a multi-variate time series classification task. . . . . . . . . . . . . . . .

3.1 Node outputs after a $\delta[n]$ input to an ESN with $N=100$, spectral radius $=0.95$, and no bias. The network experiences a decay over time due to the spectral radius being $<1 \ldots \ldots \ldots$. . . . . .

3.2 Node outputs after a $\delta[n]$ input to an ESN with $N=100$, spectral radius $=1.1$, and no bias. Note how the network node outputs do not decay after the initial input and continue to output values which fails at achieving the desired echo state property as past inputs will always affect the future state of the network. . . . . . . . . . . .

3.3 Node outputs after $\delta[n]$ input to an ESN with no bias, $N=100$, spectral radius $=0.95$, and sparsity $=0.1$. Note the oscillatory behaviour of the network nodes' output due to the addition of sparsity which differs from that in Fig. 3.1. . . . . . . . . . . . . . . . 45 
3.4 Node outputs after $\delta[n]$ input to an ESN with $U[-0.1,0.1]$ bias, $N=$ 100, spectral radius $=0.9$, and sparsity $=1.0$. Note difference in equilibrium state which differs from that in Fig. 3.1 and is more similare to the network without the desired ESP in Fig. 3.2. Due to this difference in output, the bias for the reservoirs of ESNs was set to $0 . \quad$. . . .

3.5 Structure of the deterministically constructed oscillating neural network. This construction was based off of the DNA inhibitory oscillator described $[7,10]$, where the inhibitory chemical reaction connections are mapped to negative weights and the activating chemical reaction connections are positive weights. The network can be extended to have more nodes and become a large oscillating cycle. The arrows indicate positive/activating connections and the bars indicate negative/inhibitory connections. . . . . . . . . . . . . . . . . . . 48

3.6 Node outputs from the deterministically constructed oscillating neural network when fed the $\delta[n]$ input. The network was scaled to have a spectral radius $=0.9$ and demonstrates an oscillatory behavior centered around a mean output $=0$ with a gradual decay. . . . . . . . . . .

3.7 Example of the neural oscillator as the reservoir layer with an MNIST digit input, a 0, being fed in one pixel at each time step as a scaled value of $A$ and the outputs have been shifted to have an equilibrium of 1 and normalized in the same way as in Fig. 3.8. . . . . . . . .

3.8 Example of the chemical oscillator acting as a reservoir layer with an MNIST digit input, a 0, being fed in one pixel at each time step as a scaled concentration of the chemical $A$. These chemical oscillations can then be used by a readout layer to make the classification. . . . . 
3.9 Layout of the reservoir system with the input being a one-hot encoded genome sequence. The readout layer in this case is designed to classify the input sequences based on the states of the reservoir over time as the genome is fed in. . . . . . . . . . . . . .

4.1 MSE of the ESN on NARMA-A. The blue lines represents the threshold for the baseline memoryless linear regression, $\mathrm{MSE}=0.42$. The network improves this baseline with a minimumum complexity of $N=$ 3, sparsity $=0.75$ and improves with larger networks plateauing in performance at $N=20$, sparsity $=0.1$ with an $\mathrm{MSE}=5.6 \times 10^{-6} \ldots$

4.2 MSE of the oscillating neural network on NARMA-A across different spectral radius and reservoir sizes. All of the settings performed better than the linear memoryless baseline of $\mathrm{MSE}=0.42$. The network was able to converge to a very small $\mathrm{MSE}=3.8 \times 10^{-14}$ using $N=162$ and spectral radius $=0.3$. To note is that a linear model with a memory of size 2 can perfectly solve this task. . . . . . . . . . . . .

4.3 MSE performance of the ESN on the NARMA-10 sequence across different $N$ and sparsity values, the blue line represents the linear model without memory and the redline is a linear model with input memory. With only 2 nodes the network is able to capture input memory and combination and at $\mathrm{N}=100$ the $\mathrm{ESN}$ is able to achieve better than the linear regression model with input memory even with low sparsity. . . 
4.4 MSE of the oscillating neural network on the NARMA-10 task across different $N$ and spectral radius values. The blue line represents the linear prediction model with no memory and the red line shows when the model became better than a linear model with input memory. The model shows a performance improvement as the number of nodes increases and the spectral radius causes significant difference in perfor-

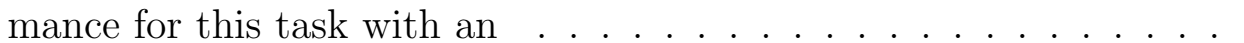

4.5 Classification accuracy for MNIST using ESNs. The ESN shows an improvement over linear classification, the blue line, with 20 nodes and a sparsity of 0.1 . There is not a significant trend in different performances for sparsity at the higher values of nodes, however at smaller node values, too much sparsity can lead to a strong dip in performance as incomplete networks can form. . . . . . . . . . .

4.6 Classification accuracy on MNIST for ESN using only the final readout value of the reservoir nodes. A significant reduction in performance was found when this constraint was placed on the model falling from $96.5 \%$ accuracy with 1000 nodes in Fig. 4.5 to $74.3 \%$ in this model. Although this does reduce the output from being $N \times 28$ to being $N \times 1$ which reduces the readout complexity. The ' $\mathrm{X}$ ' marks no accuracy value at the higher node values. . . . . . . . . . . . . . . . . .

4.7 Classification accuracy across the different parameters using the neural oscillating network. The accuracy improves as the size of the network grows crossing the blue line, which indicates the threshold for a linear classifier, when $N=30$. There is a significant trend at the larger graph size that having a larger spectral radius improves the performance . 
4.8 Classification accuracy of the ESNs on the splice junction gene sequences across the different sparsity and $N$ values. Increasing network size to above $N=5$ can improve performance above the linear baseline, represented by the blueline. Diminishing returns was found as the network grew larger. . . . . . . . . . . . . . . . .

4.9 Confusion matrix for the splice junction gene sequences of the ESN with $N=10$, sparsity $=1.0$. The predicted class is on the $\mathrm{y}$-axis and actual is listed on the $\mathrm{x}$-axis, the columns are normalized and sum to 1.0. The intron/exon junctions appear to be the most difficult to classify and get about equally confused with the other classes. . . .

4.10 Classification accuracy for the oscillating neural network on the splice junction gene sequences across the different parameters of spectral radius and nodes. All of the settings performed as well as the linear model once the variation is accounted for. . . . . . . . . . . . . 75

4.11 Classification accuracy for the ESN on the dengue virus genome subsequences. The blue line indicates linear model classification threshold. The ESN able to extract meaningful features from the 200bp sequences with $N=30$ and sparsity $=0.1$, the performance was best at $N=100$ while at higher $N$ values the network seemed to suffer from being caught in a local minimum when training the output and had higher variation which may be solved by adjusting hyperparameters such as adjusting batch size. . . . . . . . . . . . . . .

4.12 Confusion matrix showing the performance of a ESN with $N=100$, sparsity $=1.0$ on classifying the beginning 200bp of the dengue genome sequence. This shows that there may be similarities between DENV-2 and DENV-3 which are more difficult for this classifier to identify. . . 78 xiv 
4.13 Classification accuracy for DV serotype across the different oscillating neural network sizes and spectral radii. The blue line indicates the threshold for linear classification. The network performs best with the larger nodes and the highest spectral radius. . . . . . . . . . . . . . 79 


\section{Introduction}

\section{$1.1 \quad$ Goals and Motivations}

Within the body, our cells are capable of responding to environmental cues and other chemical inputs. The way in which we understand the processes of these complex biological networks can be informative both in our attempts at modeling and to utilize and program them for our desired purposes. These biological networks have been explored using different paradigms including a reductionist model of differential equations, combinatorial modeling, and neural networks [11].

Our body's cellular structure, organization, and complexity are encoded in our DNA molecules, a sequence of the 4 different nucleotides: adenine, thymine, cytosine, and guanine. Research now exists exploring the use of these very same molecules for implementing computation. DNA computing has shown promise due to its ability to perform computations in parallel at the molecular level and has been demonstrated on computing tasks such as Hamiltonian path [12], 3-SAT solver [13], and pathogen classification based on gene expression [2]. These molecular circuits are constructed using DNA strands that bind with other complementary strands and therefore need to be orthogonal to one another in order to prevent crosstalk [14]. A key issue is that

as the scale of these circuits increase, the orthogonality decreases limiting the size and complexity of these circuits [15].

In order to address this scale-up issue, we propose here an abstraction of these molecular circuits into a paradigm known as reservoir computing [16]. These reservoir computing networks consist of a fixed dynamic reservoir, which extracts features from 
the input, and a final linear readout layer which can be adapted for the specific task.

Certain components of computation have already been implemented using DNA logic circuits. In Fig. 1.1, research works on this concept are shown with the corresponding machine learning model as the top layer and labeled along the arrows are the works that have demonstrated their implementation using a chemical substrate.

The aim of this research is to explore some methods of bridging the gap between reservoir computing and its relation to chemical implementation. This could result in an improved scaling up of these networks to handle more complex tasks. It also may even be that some organisms use a form of chemical reservoir computing for gene regulation [17].

A benefit of this reservoir computing methodology is that it may be possible to establish a chemical system with rich dynamics which acts as the reservoir and then only the final linear readout needs to be tuned to the particular task.

Implementing more complex systems in a DNA circuit will be more difficult, therefore it is advantageous to identify a trade-off in terms of size and complexity of the circuit for solving a particular task. This research will explore a form of reservoir computing known as echo state networks in order to try and find small sparsely connected reservoirs which can perform well at a task. This minimizes their complexity improving their potential for physical implementation as this abstractly represents a smaller amount of chemicals and reactions which would need to be carried out by the dynamical physical reservoir system.

These physical reservoirs can be described using chemical reaction networks (CRN) which represent graphically each chemical and their reaction pathways with other chemicals in the network. Previous work has shown that these CRNs can be implemented using DNA circuits [8] and in particular a 3-way oscillator [9]. These chemical oscillators have been explored for their potential use as a reservoir $[7,10]$. 
(1).png (1).png

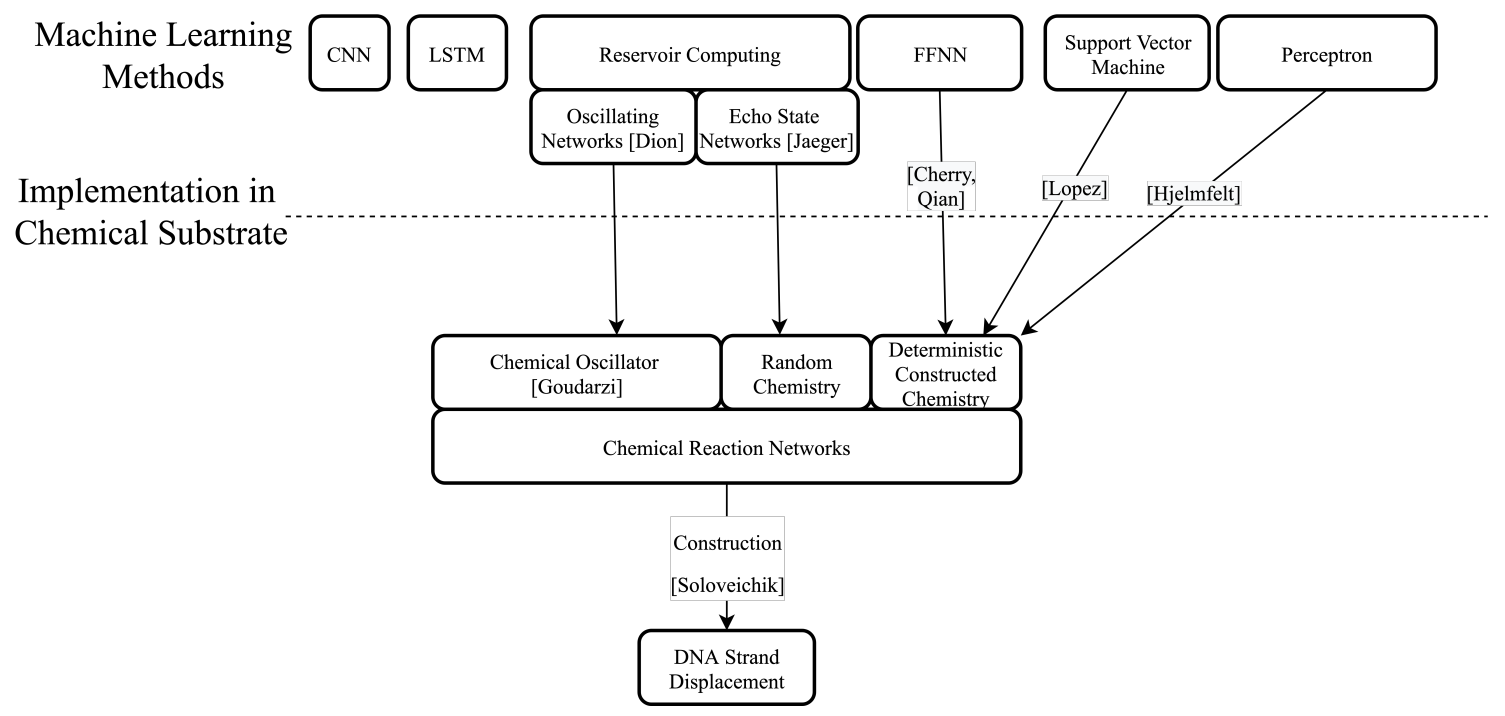

Figure 1.1: Showing some machine learning methods and previous work which has mapped these methods of machine learning onto a chemical substrate. The names on the arrows are references to previous research which has been done to bridge the gap between the mathematical machine learning methods and their implementation in chemistry Hjelmfelt [1], Lopez [2], Cherry [3], Qian [4]. The aim of this research is to explore some methods of bridging the gap between the machine learning methods in Reservoir Computing such as oscillating networks, Dion [5], and echo state networks, Jaeger [6], and their chemical implementation such as by using chemical oscillators, Goudarzi [7]. This could result in an improved scaling up of these networks to handle more complex tasks. Chemical reaction networks can be constructed using DNA strands and methods described [8]

Pathogen detection is a rising issue in today's globalized world. This coupled with the explosion of genomic data, is why the task of genome classification was explored in this research. Specifically the dengue virus genome is used as a target for classification. The potential implementation of these networks using DNA allows for the possibility of the input to be direct viral genome sensing. 


\subsection{Challenges}

Use of these reservoirs as a model of dynamic chemistry can be useful in order to overcome issues of crosstalk and difficulty training the physical systems. Crosstalk is the unwanted transfer of signals between different communication pathways or channels. Organisms are able to compensate for this crosstalk, however this can still be an issue in the construction of synthetic chemical reaction networks. As these networks become larger and more complex, there is an increased possibility of interference between different pathways.

It can also be difficult to determine the exact kinetics of DNA reactions and may in the actual chemistry inside the cell, in situ, be different due to the stochastic nature of their reactions [8]. Many assumptions in theoretical models such as the solution being well mixed or the probability of a reaction taking place can be different depending on the environment.

\subsection{Contributions}

In this thesis I have made the following contributions:

- Explored the use of echo state networks for the use of multivariate sequence classification for dengue genome classification task achieving $96.4 \%$ accuracy on 200bp subsampled segments of the genome. See Section 4.2.3 for more details

- Identify the minimum complexity echo state network which provided an improvement on MNIST over the linear classification baseline of $92.5 \%$ which could be improved with an echo state network of size $\mathrm{N}=20$ and sparsity $=0.1$. Section 4.2.1 for details. 
- Explored the use of an oscillatory recurrent neural network as a fixed hidden layer which was able to improve performance on the sequence prediction tasks above a linear model with input memory for NARMA-10 from MSE $=1.6 \times 10^{-3}$ to $1.1 \times 10^{-3}$. This network's performance was characterized for different sizes and spectral radius as well. See Section 4.1.2 for details.

- Achieved near best reported performance for the splice junction gene sequence dataset for 10-fold cross-validation using echo state networks with an accuracy of $96.8 \%$. See Section 4.2.2 for details.

- Evaluate the simulated use of a chemical oscillator as a reservoir layer for sequence prediction tasks. Showing that these chemical oscillators can improve performance above the memoryless linear baseline for NARMA-A from $\mathrm{MSE}=0.42$ to 0.34 . and for NARMA-10 from $\mathrm{MSE}=8.8 \times 10^{-3}$ to $6.6 \times 10^{-3}$. See Section 4.1.1 and Section 4.1.2 for details.

- Evaluate the simulated use of a chemical oscillator as a reservoir layer for the MNIST dataset achieving 90.6\% classification accuracy. See Section 4.2.1 for details.

- Prepared tutorials for others in the lab for reading and encoding genomic data files from the splice junction gene sequences dataset. Along with examples for creating echo state networks using PyTorch and Keras at https://github.com/Neighborc/tlabTutorials.

- Presented a research poster at the 2019 International Conference on DNA Computing and Molecular Programming in Seattle, WA, where I published a peerreviewed abstract for my poster in the conference poster proceedings. Title: "Reservoir Computing for Genome Sequence Classification." 
- Presented a research poster at the Portland State University Student Research Symposium in Spring 2018 on the topic of using recurrent neural networks to compute hamming distance on gene sequences. Title: "Using Reservoir Computing to Build a Robust Interface with DNA Circuits in Determining Genetic Similarities Between Pathogens." 


\section{Background}

\subsection{Neural Networks}

The human brain is our most personal reference we have for creating an intelligent system. One way of modeling the brain is as an information processing system which takes as input the sensory impulses, transforms and processes them using biological neurons, and then generates outputs of action into the world. Within the brain the neurons communicate across synapses by generating electrochemical impulse spikes known as action potentials. The neuron then accumulates this current with a small leak due to diffusion. If a neuron receives enough current to be above a certain threshold, it then fires a spike. The brain is capable of changing these connection weights between each of its neurons and a model of Hebbian learning can be applied [18]. While this may be an oversimplification, it can be used to generate ideas of how we might construct an information processing system and ways in which to update the system in order to improve performance at a task.

\subsubsection{Feed Forward Neural Networks}

From this simple model of the brain's neurons, a mathematical model for an artificial neuron, called the perceptron, was developed by McCulloch and Pitts [19]. This perceptron functions by summing the inputs according to a weights matrix and then passing this summation through a threshold unit. Later, different activation functions were used in order to achieve non-linear transformation and differentiability such as logistic function, hyperbolic tangent, or rectified linear unit. A layout of this 
perceptron can be seen in Fig. 2.1. Such a model is able to learn linear separations such as the logical functions of AND as well as OR, but fails with the task of XOR and NXOR. This limitation however can be overcome through the use of multi-layered perceptrons (MLP), in which more perceptrons are added as well as increasing the number of layers in the hidden layer of the network which can be seen in Fig. 2.2.

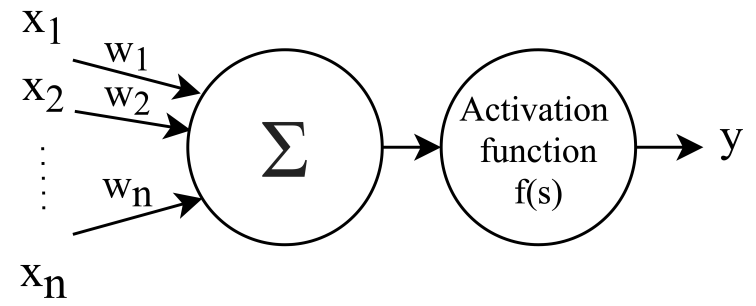

Figure 2.1: The perceptron model which takes the weighted summation of the inputs and then passes them through an activation function to generate an output.

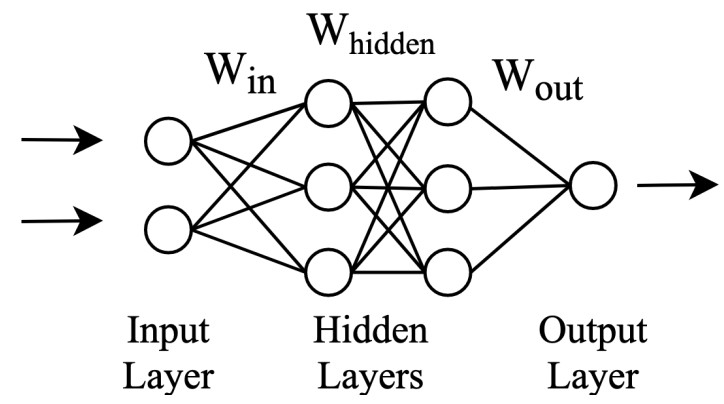

Figure 2.2: A multi-layered perceptron network, the input is fed into the input layer and passed forward through the hidden layer then on to the output layer, each of the nodes within the hidden layer are a perceptron. Due to its multi-layered structure it is capable of solving non-linear separation problems.

This is known as a feed forward neural network (FFNN). This type of neural network, passes all of the values forwards and is deterministic in its calculations. When the target output is known, the network can then go through a training process, known as supervised learning, in which the weights within the network can then be adjusted according to the backpropagation of the error [20]. 


\subsubsection{Gradient Descent}

An iterative process of gradient descent can be used to train the weights to a practical solution for the problem. The gradient descent equation consists of evaluating the gradient of the loss function with respect to the weights and the error from a teacher

signal. This is then used to update the weights using backpropagation and a selected learning rate.

$$
W_{t+1}=W_{t}-\eta \Delta_{W_{t}} J(W)
$$

Where $W$ is the weight matrix, $\Delta_{W_{t}}$ is the gradient of the error with respect to the weights, $J(W)$ is the error based on a cost function, and $\eta$ is the learning rate.

The selection of this learning rate, $\eta$, is important for rate of convergence as too high of a learning rate can lead to lack of convergence whereas too small of a learning rate can lead to very slow convergence. The use of stochastic gradient descent (SGD) can greatly increase the speed of convergence and utilizing batching can reduce the variability in the gradient direction [21].

\subsubsection{Activation Functions}

A variety of different functions have been used for the activation function. After the input into the artificial neuron is summed, it is then passed through a non-linear transformation function before being passed on to the next artificial neuron in the network. Three common activation functions which are used and were explored in this research are the logistic or sigmoid function, hyperbolic tangent (tanh), and Rectified Linear Unit (ReLU). A diagram of each of the activations is shown in Fig. 2.3 


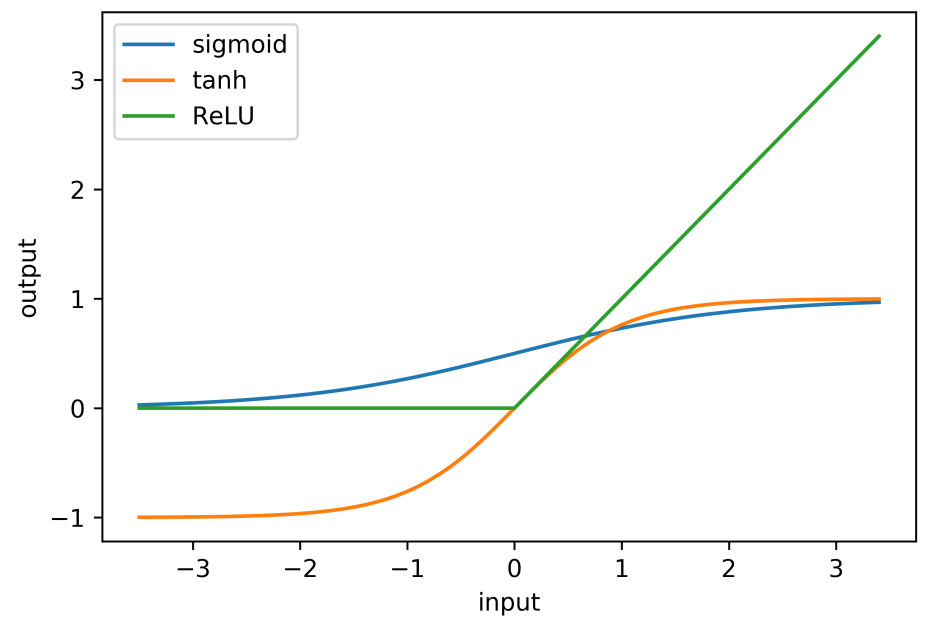

Figure 2.3: The common non-linear activation functions for neural networks of sigmoid, tanh, and ReLU.

Equations for each are as such:

$$
\begin{aligned}
\operatorname{sigmoid}(x) & =\frac{1}{1+e^{-x}} \\
\tanh (x) & =\frac{e^{x}-e^{-x}}{e^{x}+e^{-x}} \\
\operatorname{ReLU}(x) & = \begin{cases}x & \text { if } x>0 \\
0 & \text { otherwise }\end{cases}
\end{aligned}
$$

Each of these functions are differentiable which allows for the use of backpropagation and gradient descent in updating the weights to correct for the error.

\subsubsection{Convolutional Neural Networks}

Another popular architecture is convolutional neural networks (CNN). These models have been used especially on 2D computer vision tasks [22,23]. An advantage of these types of networks is that they better incorporate aspects of spatial arrangement by 
looking at neighboring information. In the case of images, the convolutional layer consists of creating a $K \times K$ filter which then strides across the image matrix values. This output is combined with the outputs of other filters to create a feature map often followed by a pooling layer which then feeds into a final FFNN output layer which classifies the input. An introductory guide with animated visualizations can be seen on the Stanford CS231n website [24].

Convolutions can also be performed on 1D or 3D data. The use of "convolution" in these neural networks is a bit of a misnomer in that the actual mathematical transformation being performed is cross-correlation since the filter itself is not flipped.

\section{Transfer Learning}

For transfer learning is that the CNN has already been trained on a dataset and only the final readout layer is trained [25]. For CNNs there is a large number of fully trained models available to download and access through frameworks like PyTorch. These models have been trained and fine tuned for a particular task such as ImageNet1000. Within the model there are a large number of parameters which need to be tuned, however for transfer learning only the fully connected output layer is trained for a particular task. The pre-trained model is capable of extracting meaningful features which can be used to effectively differentiate between the desired classes.

It may be possible to apply this transfer learning using pre-trained models from other applications of CNNs such as for DNA classification [26].

\subsubsection{Recurrent Neural Networks}

This is predominantly the architecture which will be explored in this research. The main difference between this and the FFNN is that the output from each of the nodes is then fed back into the network according to a connection matrix. 
This results in a kind of rolled out over time feed-forward neural network which can be seen in Fig 2.4. In order to train this model, the gradient needs to be passed backwards through previous time points. This results in an issue known as the vanishing or exploding gradient problem when the scaling of the gradient in the backward pass is less than 1 or greater than 1 respectively. [27]

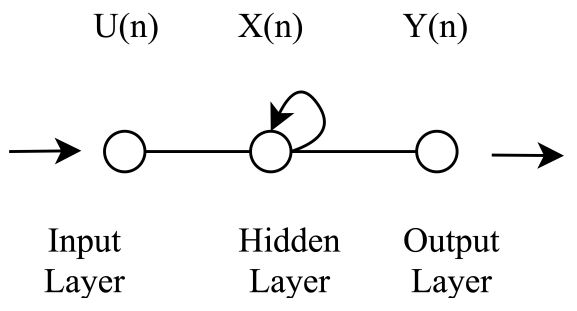

Figure 2.4: Simple RNN node with the recurrent connection in the hidden layer. Note the output node can be connected to the hidden layer node's output across time thus adding to the time dependencies. A typical activation function for the recurrent node is tanh.

Other node architectures which can be used include the long-short term memory (LSTM) networks which keep track of 2 hidden states and contain gates within the node which are used to control information to store, forget, and incorporate into the output [28]. They overcome the vanishing gradient problem by setting the backward pass scaling $=1$ and passing it through the long-term memory cell. The benefits of these over simple RNNs and how they can better handle long term dependencies have been descibed [29]. Other descriptions of LSTMs include this blog post [30].

\subsection{Reservoir Computing}

Reservoir computing explores the idea of using a dynamical system as a projection kernel in order to improve linear separability of the task [16].

Examples of dynamical systems include mathematical models of a swinging clock pendulum and the rise and fall of predator and prey population in an environment. 
The current state of a dynamical system can be represented using a vector which represents a point in a state space. The current state is then progressed to a future state using a mathematical model with a function which can be either deterministic or stochastic and can show chaotic outcomes [31]. Sensitivity to initial conditions in dynamical systems is explored in chaos and has been explored in RNNs [32].

For reservoirs using RNNs, the echo state networks (ESNs) were proposed which consisted of simple recurrent neural networks which were randomly connected and had their weights scaled to ensure an echo state property (ESP) which essentially guarantees that eventually the original state of the network will decay over time end the final output of a large finite time series will not be affected by initial states [33].

This computing paradigm, proposed in 2001, arose as a means of leveraging the benefits of recurrence and non-linearity within the model without requiring the extensive compute power required for training the network and circumvented the issue of vanishing and exploding gradient which is involved in back propagation through time. Other methods proposed to simplify training include backpropagationdecorrelation [34] which only trains the output layer of a RNN as well as a feedback from the output to the hidden layers.

A diagram of the layout of a reservoir can be seen in Fig. 2.5. In the case of an echo state network the internal nodes consist of perceptrons with a tanh activation and recurrent connections. The number of nodes within the reservoir can much larger than shown in the figure with some implementations having tens of thousands of nodes [35].

The internal dynamics of the reservoir are controlled with $W_{\text {res }}$ which is an $N \times N$ matrix indicating the strength of connection and sparsity of connection between the nodes. The equation used for updating the node outputs, where $X[n]$ represents the 


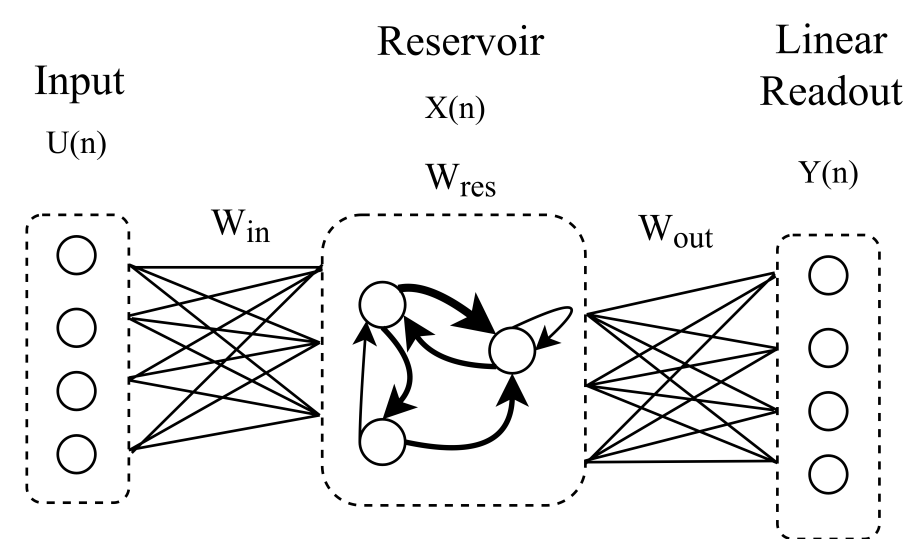

Figure 2.5: Layout of a Reservoir. The input is connected with the randomly initialized weight matrix, $W_{i n}$, into the reservoir whose fixed connection matrix, $W_{\text {res }}$, has been randomly initialized and fixed, the output states of the reservoir layer are then passed to a linear readout layer whose connection matrix, $W_{\text {out }}$, can be updated and trained for the particular task.

state of the reservoir at time step $n$.

$$
\begin{aligned}
X[n+1] & =f\left(W_{i n} U[n]+W_{\text {res }} X[n]\right) \\
Y[n] & =g\left(f(X[n]) W_{o} u t\right)
\end{aligned}
$$

Where $f()$ and $g()$ are activation functions, $X[n]$ is the state of the reservoir, $U[n]$ is the input, and $Y[n]$ is the final output. Also to note is that there are implicit bias nodes for each of the layers.

When initializing the weights in $W_{\text {res }}$, the ESP is desired for the necessary dynamics for computation. The echo state property can be achieved for all inputs using the methodologies in [36] which essentially consist of:

1. Initialize the internal reservoir weights with a uniform distribution $U[-1,1]$.

2. Scale the weights by dividing by the absolute value of the maximum eigenvalue and multiplying by the desired spectral radius $<1$. 
The largest eigenvalue within the $W_{\text {res }}$ is known as the spectral radius and gives a sense of the length of memory of the network. A spectral radius of less than one, ensures a fading memory and prevents saturation of the node outputs. A typical default value for spectral radius is 0.9 although this value can be tuned to be more task specific. Sparsity is also allowed within the network and can allow for improved computational speed as the reservoir grows larger. Some other research into optimum settings include evaluating the Lyapunov exponent and computing on the edge of chaos [32].

Initial work was promising in the capabilities of ESNs to perform effective time series analysis [16]. However, as computation has increased by an exponential amount the training time for other neural network architectures, such as LSTMs and CNNs, has shrunk and they have become more easily accessible for sequence modeling [37]. However, a possible advantage of reservoir computing is in its potential for physical implementation using devices such as memristors or chemistry [38]. Reservoir computing has potential to be effectively implemented using DNA oscillator circuits or some other dynamical chemical system [7].

Reservoir computing is an idea which doesn't use the methodology of gradient descent within the network. This is relevant in cases where there is not necessarily a good means of implementing a backpropagation into the model. This can be the case when using randomly implemented chemistry, memristors, and self assembled nanowires [38].

Separately another method for this idea of reservoir computing was proposed using a different style of nodes for the reservoir layer. A brain inspired spiking neural network was used with the concept of liquid state machines (LSM) [39]. This name is derived from the idea that a dynamic liquid, such as a bucket of water, could be used for computation. In this case the "liquid" is a network of spiking neurons which 
have been randomly connected. These LSMs were shown to have adequate separation property and the approximation property. Some of these spiking neural networks have been built on a chip such as Intel's Loihi [40].

Examples of other previous research related to reservoir computing include random kernel projection [41], using large randomly connected FFNNs in extreme learning [42], weight agnostic networks [43], and training of LSTMs by genetic algorithm [44].

\subsubsection{Readout Layer Training}

After the reservoir comes the final readout layer whose output matrix can be trained using supervised learning to match the desired output target. Because only the final output layer is trained, the training complexity of the system is greatly decreased and the speed of training increases due to having much fewer weights to train [34]. This final layer is typically constrained to being linear although it can also be a MLP [45].

For the readout layer, a distinction between only using the final output from the reservoir or the entire time series of the nodes can lead to very different accuracies [46]. By using the value of the node outputs across time, the computational complexity is greatly increased for the final readout layer. For example in the case of a sequence of $T$ steps and a reservoir with $N$ nodes, the final readout layer will be trained on either a vector of size $\mathbb{R}^{N}$ or in the case of concatenating the node states for the final readout it will be trained on vector of size $\mathbb{R}^{N \times T}$. In the case of long sequences, large value of $T$, this will greatly increase the complexity. A large improvement in the performance of the model, however, can be found by incorporating this into the final readout layer. This is due to the fading memory state of the model as well as the incorporation of further sigmoidal functions into recurrent states from the beginning of the input sequence.

A solution which is a compromise between the two ideas can either be to evaluate 
the average output state of the nodes over a certain period of time or subsampling the output states of the node [47]. Additional methods have been explored for reducing the dimensionality of the reservoir states using principle component analysis [45].

\subsubsection{Linear Regression}

When mapping the reservoir outputs to a value, linear regression can function as a supervised machine learning algorithm which can be modeled as:

$$
h_{W}(x)=W_{0}+W_{1} x_{1}+\ldots+W_{n} x_{n}
$$

In which $h_{W}$ is the hypothesis, $n$ is the number of features, $m$ is the number of training examples, $x$ is the input vector, and $y$ is the target output value in the following equations.

The aim of the linear regression is to find the weights, $W$, which map the input vector to the desired target output value. The effectiveness of these weights can be evaluated using a cost function and then the values of the weight matrix can be adjusted such that this cost is minimized.

$$
\underset{W}{\operatorname{argmin}}(J(W))=\frac{1}{2 m} \sum_{i=1}^{m} \operatorname{cost}\left(h_{W}(x), y\right)
$$

For mean squared error this cost function is:

$$
\operatorname{cost}\left(h_{W}(x), y\right)=\left(h\left(x^{(i)}\right)-y^{(i)}\right)^{2}
$$

This is a least squares problem which is trying to minimize the mean squared error 
of the approximated value, $\hat{y}$, to the target value, $y$. This can be analytically solved.

$$
\begin{aligned}
& \underset{W}{\operatorname{argmin}}\|X W-y\|^{2} \\
& \quad W=\left(X^{T} X\right)^{-1} X^{T} y
\end{aligned}
$$

The Moore-Penrose pseudo inverse matrix, $\left(X^{T} X\right)^{-1}$, is used in order to calculate the values of this solution in what is known as the normal equations.

\subsubsection{Ridge Regression}

Another means of applying linear regression but with a regularization term is by using ridge regression or Tikhonov regularization. Ridge regression is designed to avoid collinearity in which near linear relationships are present among the independent variables [48].

Ridge regression minimizes the least squares value along with an additional penalty term for the square values of the weights which is known as L2-regularization.

$$
\begin{gathered}
\underset{W}{\operatorname{argmin}}\|X W-y\|^{2}+\lambda\|W\|_{2}^{2} \\
W=\left(X^{T} X+\lambda I\right)^{-1} X^{T} y
\end{gathered}
$$

The term $\lambda$ is a tunable regularization term which is a means of constraining the weights of the model in order to reduce the overfitting of the model and improve generalization.

This value $\lambda$ can be selected for the task using $\mathrm{k}$-folds cross validation on the training sequence and then using that value on the test sequence.

An additional methodology to note is the elastic net regularization paradigm which combines the $\operatorname{LASSO}(L 1$ regularization) with the $L 2$ regularization. In this case the 
$L 1$ norm value is the mean absolute value of the weights. The $L 1$ norm can be used to try and induce sparsity in the weights.

$$
\underset{W}{\operatorname{argmin}}\|X W-y\|^{2}+\lambda_{1}\|W\|_{1}+\lambda_{2}\|W\|_{2}^{2}
$$

This elastic net regularization may improve performance, but it was not typically mentioned in the reservoir computing literature so in order to make the results more comparable the ridge regression and least squares solutions were used. The ridge regression was used when there was a significant difference between the training and the test accuracy. A large difference between the training and test accuracies can indicate that overfitting is occurring [21]. This can result in a bias-variance tradeoff. Bias, in this context, is how close the model is to the ground truth prediction and the variance is essentially how far off the model will be for generalization. The goal is to have good fit to the training data without overfitting and thus causing the generalization to new data to suffer. By introducing regularization the complexity of the hypothesis space is reduced and the weights constrained which aids in improving the model's generalization.

\subsubsection{Classification Readout Layer}

The learning task of classification has the aim of mapping a particular set of input features into a set of output classes. Examples of predicting a class from an input include classifying a picture as being either a cat or dog, determining what digit has been spoken on an audio recording, or identifying whether a movie review is negative or positive.

When the data given has the class labels, we can apply supervised learning algo-

rithms such as gradient descent. Here the use of stochastic gradient descent works 
very effectively in conjunction with the tools of logistic regression. For a binomial classification the final output is typically a sigmoid function whereas for a multi-class problem a final output layer of softmax which returns the probability of each class can be used.

$$
\operatorname{softmax}(x)=\frac{e^{x}}{e^{x}+e^{y}+e^{z}}
$$

A support vector machine (SVM) output layer can also be used which attempts to maximize the margins of the decision boundary and uses a hinge-loss function, this method has been used on biological data before [3,49] and was explored as an output layer in this research.

It is also possible for the output target to be a one-hot encoded sequence which is then trained using the sequence prediction techniques to match the input to the target sequence. The average value of this output could then be used as the classification class.

Multi-class classification can be solved using the one-to-many method. Which for example would be the case in MNIST where the classifier learns to classify "8" and "not 8" it then using a categorical cross-entropy loss function which can be used to evaluate all of the classes together. The reason behind using softmax for our research is due to it being able to be mapped to the winner-take-all DNA network which was used in a chemical classifier [3] and the ability of it to be trained effectively using gradient descent which can handle larger datasets well.

\subsubsection{Error Measures}

\section{Value Prediction Loss Metrics}

The error metric which was used to train the readout layer and evaluate the performance was the mean squared error (MSE) which was the loss function to minimize. 
The normalized root mean squared error (NRMSE) was used in some previous work. Additionally the normalized mean squared error (NMSE) was found in some literature [50]. The equations and relations of these different error functions are presented below.

$$
\begin{aligned}
M S E & =\left\langle(\hat{y}-y)^{2}\right\rangle \\
N M S E & =\frac{M S E}{\sigma_{y}^{2}} \\
N R M S E & =\frac{\sqrt{M S E}}{\max (y)-\min (y)}
\end{aligned}
$$

Where $\hat{y}$ is the approximation, $y$ is the target, $\sigma_{y}$ is the standard deviation, and \langle\rangle denotes the mean. The main plots for this research will be in MSE and when comparing to previous work their error metric is converted into MSE.

\section{Classification Loss Functions}

When differentiating between two classes, the loss function of binary cross-entropy can be used. In this case, rather than attempting to match the output of the network to reduce the mean squared error, a binary cross entropy loss can be used. Binary cross entropy:

$$
\text { Binary cross entropy: } \operatorname{loss}=-(y * \log (p)+(1-y) \log (1-p))
$$

For multi-class the loss function of categorical cross entropy for one-to-many can be minimized. This calculates a separate loss for each class label and then sum the result.

$$
\text { Categorical cross entropy: loss }=-\sum_{c=1}^{M} y_{o, c} \log \left(p_{o, c}\right)
$$


Where $M$ is the number of classes (dog, cat, fish), log is the natural log, $y$ is a binary indicator (0 or 1$)$ indicating if class label $c$ is the correct classification for observation $o$, and $p$ is the predicted probability observation $o$ is of class $c$.

The goal for the end result is to generate a model which can generalize well to other data and achieves a high accuracy. It is also important to evaluate the benchmark performance with human classification skills.

\subsection{Chemical Reaction Networks}

Chemical reaction networks $(\mathrm{CRNs})$ are a representation of the concentrations and the reaction rates of different chemicals in a solution. These can be represented as graphs with each node being a chemical and the edges being mapped to reaction pathways. Their reactions can be modeled using differential equations based on their concentration and reaction rate values.

An example of a CRN is the Lotka-Volterra (Predator-Prey) equations in which there are only three chemicals: a fuel $(F)$, a prey chemical $(X)$, and a predator chemical $(Y)$. Their reactions are represented below.

$$
\begin{gathered}
F+X \stackrel{\mathrm{k} 1}{\rightarrow} 2 X \text { prey reproduces itself using fuel } \\
X+Y \stackrel{\mathrm{k} 2}{\rightarrow} 2 Y \text { predator consumes prey and reproduces } \\
\quad Y \stackrel{\mathrm{k} 3}{\rightarrow} 0 Y \text { predator breaks down }
\end{gathered}
$$

Each of these reactions are characterized by their concentration and a reaction rate, $k$, which indicates the speed at which the chemicals react. For these equations

the settings of $F=k 1=k 2=k 3=1$ work for generating oscillations which can be 
seen in Fig. 2.6.

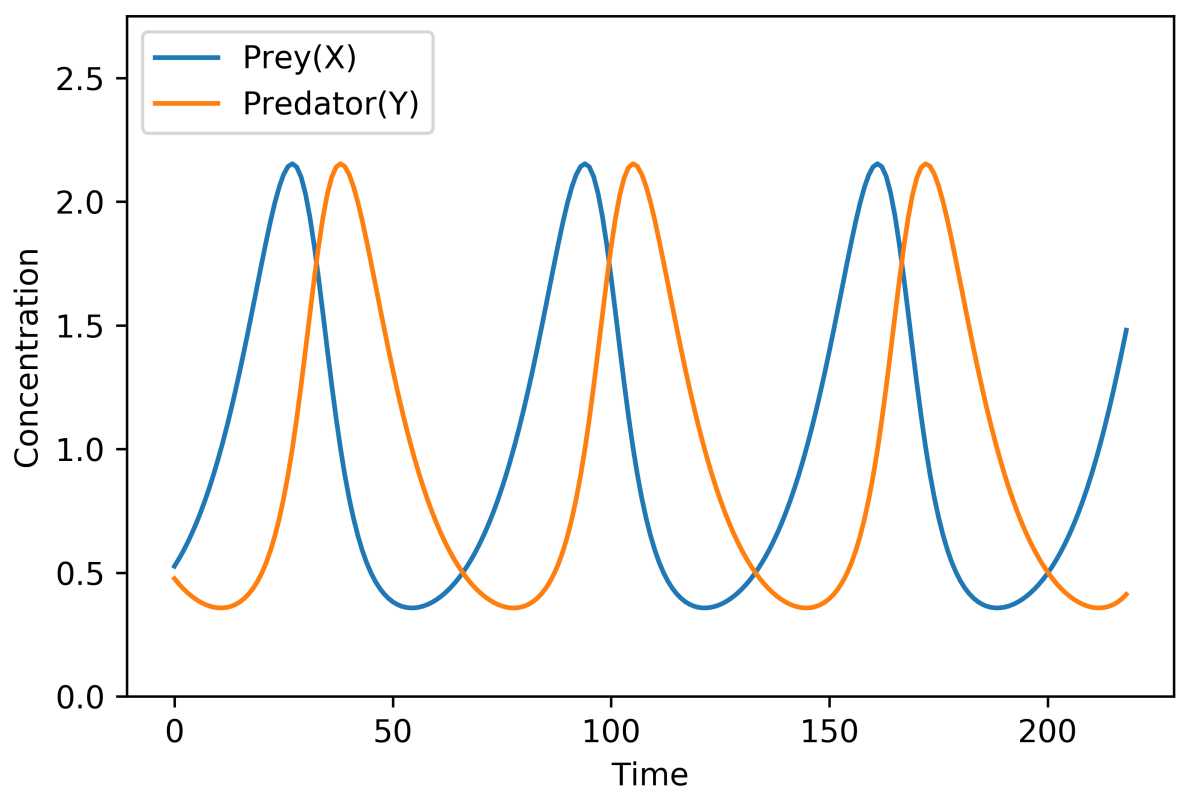

Figure 2.6: Chemical oscillations present in the Lotka-Volterra (Predator-Prey) reactions. Present in this system is also a chemical fuel source which is used to produce the Prey chemical, however, it is present at a large enough quantity to be held constant.

These chemical reactions can be modeled using mass-action kinetics which describe the change in concentration of these molecules over time using differential equations. Another common examples of these kinds of implementations include Michaelis-Menton [51] for describing enzyme and substrate breakdown.

When the number of chemicals in a solution is sufficiently small, around $<1,000$, mass-action kinetics as a model breakdown and a different methodology of simulation using the Gillespie algorithm may be a more accurate representation [52].

\subsubsection{DNA Strand Displacement}

A key building block of implementing these chemical networks is DNA toe-hold mediated strand displacement (TMSD) [53] which can be used to create arbitrary chemical 
reactions, making it a engineering problem of implementation rather than combinatorics $[8,9]$.

Current methodologies for DNA computing focused on modularizing these molecular circuits as boolean logic gates and cascading them together for computation [4]. Previous work has been done to show that DNA TMSD can be used to compute AND and OR gates [54].

Another key component of a classifier is using a winner-take-all system for the final readout layer which has been demonstrated using DNA [3]. If the class is trained to be the highest probability with the largest margin then the winner-take-all will be effective for the final layer output.

Some work has been done to implement machine learning algorithms using DNA. Initial work has been done to use TMSD to implement a pathogen classifier with boolean gene detection as an input to the network [2] followed by an SVM readout layer. Other methods have included constructing a weighted FFNN which compares the inputs to a template of weight concentrations which represent an average value of a class [3].

\subsubsection{Oscillating Chemical Networks}

A dynamic chemical system which has could potentially be used as a resevoir layer is a 3-way chemical oscillator [7]. An example of a 3-way chemical oscillator was demonstrated using DNA-strand displacement [9]. This dynamic system consists of the three chemicals $\mathrm{A}, \mathrm{B}$, and $\mathrm{C}$ as well as their reaction rates $\mathrm{k} 1, \mathrm{k} 2$, and $\mathrm{k} 3$. The system was constrained to $A+B+C=K$ where $K$ is a constant and they selected 
$k_{1}=k_{2}=k_{3}$

$$
\begin{aligned}
& A+B \stackrel{\mathrm{k} 1}{\longrightarrow} 2 B \\
& B+C \stackrel{\mathrm{k} 2}{\longrightarrow} 2 C \\
& C+A \stackrel{\mathrm{k} 3}{\longrightarrow} 2 A
\end{aligned}
$$

Assuming first order reactions this results in the differential equations.

$$
\begin{aligned}
& \frac{d A}{d t}=-k 1[A][B]+k 3[C][A] \\
& \frac{d B}{d t}=-k 2[B][C]+k 1[A][B] \\
& \frac{d C}{d t}=-k 3[C][A]+k 2[B][C]
\end{aligned}
$$

Using these differential equations, we can numerically solve for a set $\Delta t$ time step and generate the oscillating behavior from this CRN which can be seen in Fig. 2.7.

This chemical oscillator is explored in these experiments for use as a reservoir layer where the input into the reservoir is a scaled concentration of chemical $A$ and a linear readout layer with the chemical concentrations of $A, B$, and $C$ are used to train $W_{\text {out }}$.

It is also interesting to note that these types of oscillatory networks can also be expanded to have a larger number of elements, going up to much larger values of $N$. The sensitivity of these chemical oscillators to perturbations has been explored in previous work [9]. 


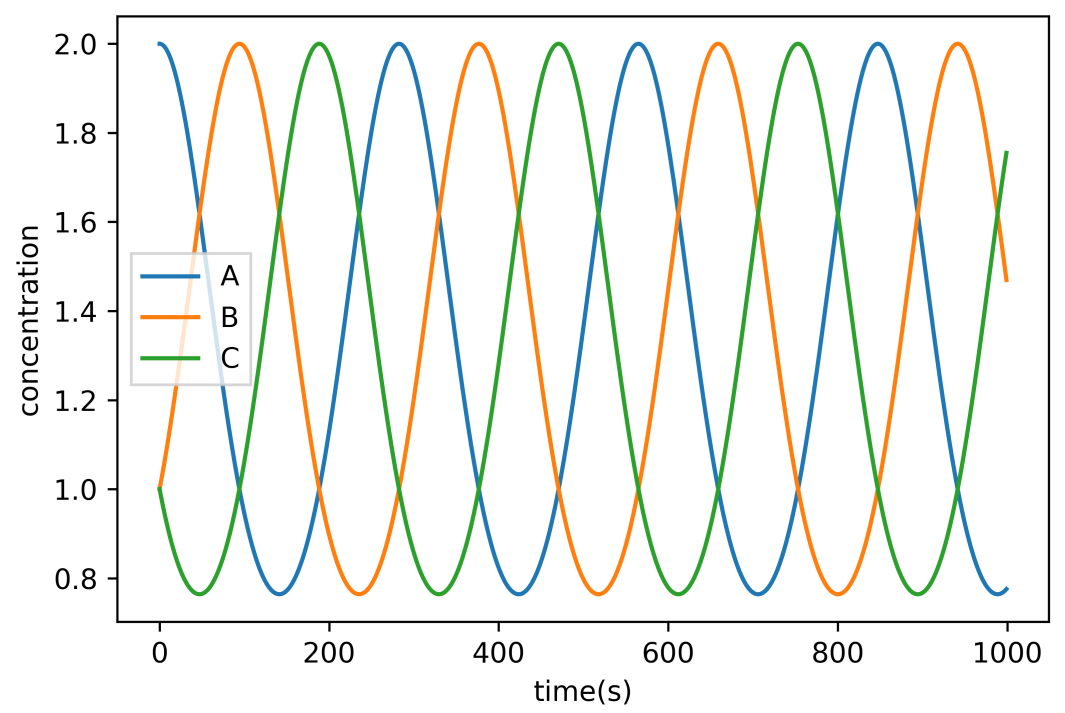

Figure 2.7: Oscillatory behavior from the displacillator chemical reaction equations which have been implemented using DNA strand circuits [9]. These reactions are explored as a reservoir layer where the input into the system is a scaled concentration of chemical $A$ and a linear readout layer of the chemical values is used.

A 4-way oscillator can be constructed using a similar ordering with these equations:

$$
\begin{aligned}
& A+B \stackrel{\mathrm{k} 1}{\longrightarrow} 2 B \\
& B+C \stackrel{\mathrm{k} 2}{\longrightarrow} 2 C \\
& C+D \stackrel{\mathrm{k} 3}{\longrightarrow} 2 D \\
& D+A \stackrel{\mathrm{k} 4}{\longrightarrow} 2 A
\end{aligned}
$$

We explored using this 4-way oscillator for genome sequence classification tasks so that each of the 4 nucleotides could be input to the system as a scaled version of each of the four chemicals. This CRN is also constrained to have $A+B+C+D=K$ and $k_{1}=k_{2}=k_{3}=k_{4}$. 
In previous work for these chemical oscillators, the PENDNA toolbox was used for

simulation [10] and an evolutionary genetic algorithm, based off of NeuroEvolution of Augmenting Topologies, was used in order to optimize the network according to the task. They describe a circular recurrent neural network which can be used as an equivalent to the chemical oscillatory behavior which is described [7].

Another key building block in reservoir computing which was demonstrated in CRNs was the presence of delay lines. Within a reservoir it is possible to implement a delay line via a circular graph layout which can then be fed back into the desired node.

The computational power of mechanical oscillators coupled with reservoir computing has been demonstrated for just a single recurrent node with a delay line in a task of spoken digit classification [5]. It may be possible to similarly leverage these chemical oscillators as effective reservoirs for desired tasks.

\subsubsection{Gillespie Chemical Simulation}

An issue with these types of simulations, is that they assume mass action kinetics which are present when a relatively large concentration of the chemicals are present. However, in the cell, with a small concentration of chemical molecules, stochastic simulation may be a more realistic and accurate model. The Gillespie algorithm is used in this case [52].

The reactions are based on a propensity function, essentially saying the probability of whether or not a given reaction is going to take place for a given time step. Challenges of this method include the continued underlying assumption of a well mixed reaction and does not necessarily incorporate any spatial information into the calculations. This would require a more advance model which incorporates diffusion and the capability of reactions occurring in parallel. 
This form of stochastic modeling is also much more computationally difficult due to the Monte Carlo step which needs to generate random numbers to determine the reactions and because these reactions have to be carried out in series rather than in parallel which creates a bottleneck in calculation speed.

Ways of avoiding this include the method of tau leaping, which basically calculates several reaction steps with the current molecule values before updating and is tuned to not change the reaction rates by some tolerance say around $3 \%$ [52]. This can result in a significant speed-up.

\subsubsection{K-mer Extraction}

One of the most prominent preprocessing techniques for genome sequences is a form of feature extraction known as k-mer extraction. K-mer extraction uses a sliding window along the genome and encodes the count of a particular subsequence. For example when $k=3$, the number of sequences on the genome, such as $A A A$, are counted. This results in an output vector of $64\left(4^{3}\right)$. A visual of how this process works is shown in Fig. 2.8. This avoids one of the main issues with genomic sequences which is alignment and varying lengths of the sequences [55]. Many machine learning algorithms are trained on a particular size of input and adjusting to be able to handle sequences of different length can be difficult with methods like padding zeros or truncation of the sequence causing some loss in information.

This k-mer analysis is similar to Shannon's seminal paper on communication and information theory [56]. If we are able to establish a probability distribution of each of these k-mers, we can use this statistical information to perform particular tasks such as classification, however, this preprocessing step loses much of the information which is present within the genome which comes from position. 


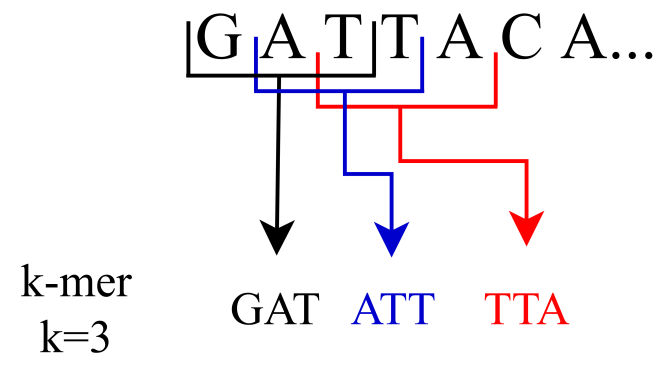

Figure 2.8: Example of how the feature preprocessing step of k-mer extraction works. A sliding window of size $k$ is moved along the genome and the count of each of short sequences of length $k$ is tracked giving a k-mer frequency distribution.

\subsection{Related Work}

In this section we present datasets being used in this research and some of the previous related work which has been done along with the benchmarks achieved.

Some these datasets are generated using a known mathematical formula and thus work well for establishing a solid baseline and allow for troubleshooting and comparisons. Also used in this research are some real world datasets which have been gathered previously; these can be biological in origin and often contain noise which may need to be filtered in the data preprocessing step.

\subsubsection{NARMA}

The nonlinear auto-regressive moving average (NARMA) dataset is a sequence which is designed to evaluate the memory capacity of the system as well as its ability to combine previous input values.

For comparison to other reservoir computing sequences we explored the NARMA Task A (NARMA-A) [7], and the NARMA-10 sequence [57,58]. 


\section{NARMA Task A}

The desired NARMA-A sequence is generated using the following equations:

$$
\text { NARMA-A: } y(t)=u(t-1)+2 u(t-2)
$$

where $u(t)$ is from $U[0,1]$. A thing to note is that this task is trivial to solve if one is given the full memory of the input values as the solution is just a linear combination. The aim for this task was to demonstrate the short term memory of the reservoir in its output states and the capacity of the readout layer to effectively combine them to match the target sequence.

The NARMA-A was previously explored using a simulated chemical oscillator circuit and the NRMSE was found to be $0.23, \mathrm{MSE}=0.16$. For this research, a comparison baseline using a ridge regression model which only sees the current input, memoryless, will be used.

\section{NARMA-10}

The NARMA-10 sequence has more long term non-linear characteristics and is generated using the following equation:

$$
\text { NARMA-10: } y(t)=\alpha y(t-1)+\beta y(t-1) \sum_{i=1}^{n} y(t-i)+\gamma u(t-n) u(t-1)+\delta
$$

Where $u(t)$ is from $U[0,0.5]$. The input to the system is the random vector, $u(t)$, the target values is $y(t)$ with the constants $\alpha=0.3, \beta=0.05, \gamma=1.5, n=10$, and $\delta=0.1$ An example of a NARMA-10 sequence is shown in Fig. 2.9

Previous work has been done using reservoir computing on the NARMA-10 task as a benchmark. The output can be calculated when given access to the inputs and 


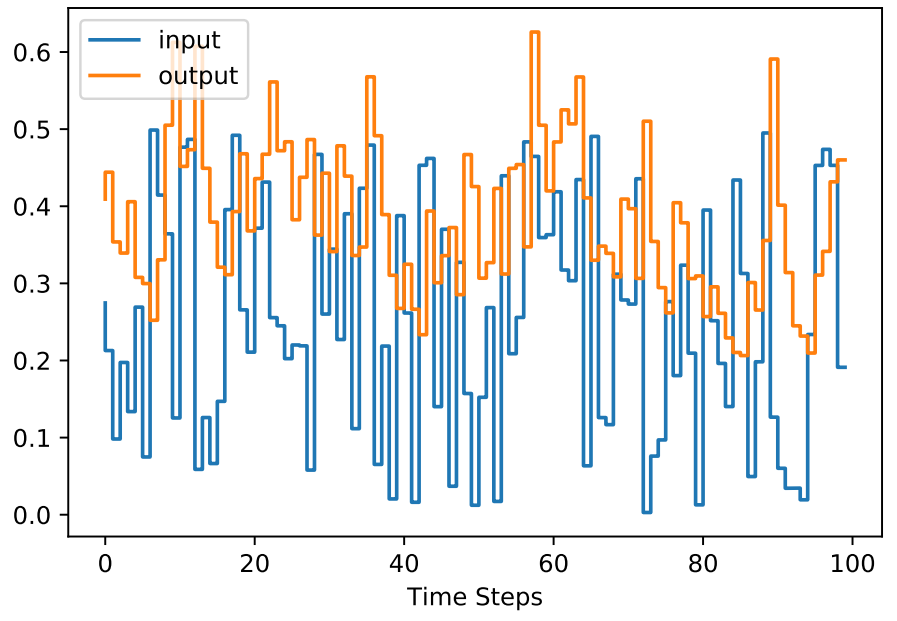

Figure 2.9: Example of the NARMA-10 sequence generated from the random vector input. From the equation it can be seen that the output is constructed from a scaled previous output, the previous input times the scaled moving average of the previous 10 outputs, a combination of previous inputs 10 steps apart, and add a constant.

the previous outputs of the series. For this problem the MSE will be evaluated for comparison with previous work $[50,57]$.

For these benchmarks the input to the system is the random uniform vector which is passed through the reservoir and then the target output is mapped using the node outputs. It can also be possible to improve the performance on this task using the memory of the previous output values of the reservoir.

Some previous reported values on this task are an MSE $=2.64 x 10^{-4}[58]$ with the settings of input scaling $=0.1, N=500$, sparsity $=1.0$, and spectral radius $=0.9$. Other work achieved an NMSE $=0.1075$, equivalent to MSE $=0.00133$, [50] with the settings of input scaling $=0.1, N=100$, sparsity $=0.18$, and spectral radius $=0.9$.

For my results, the MSE was calculated and compared to some of the converted benchmark results based on the values from the particular dataset such as $\max (y(t))$ 
and $\min (y(t))$ which is used when calculating NRMSE and the variance of the sequence which is used for the NMSE.

It is interesting to note that the NARMA-10 dataset has some long term dependencies due to its formulation. A way of seeing this is by plotting the autocorrelation of the sequence in Fig. 2.10.

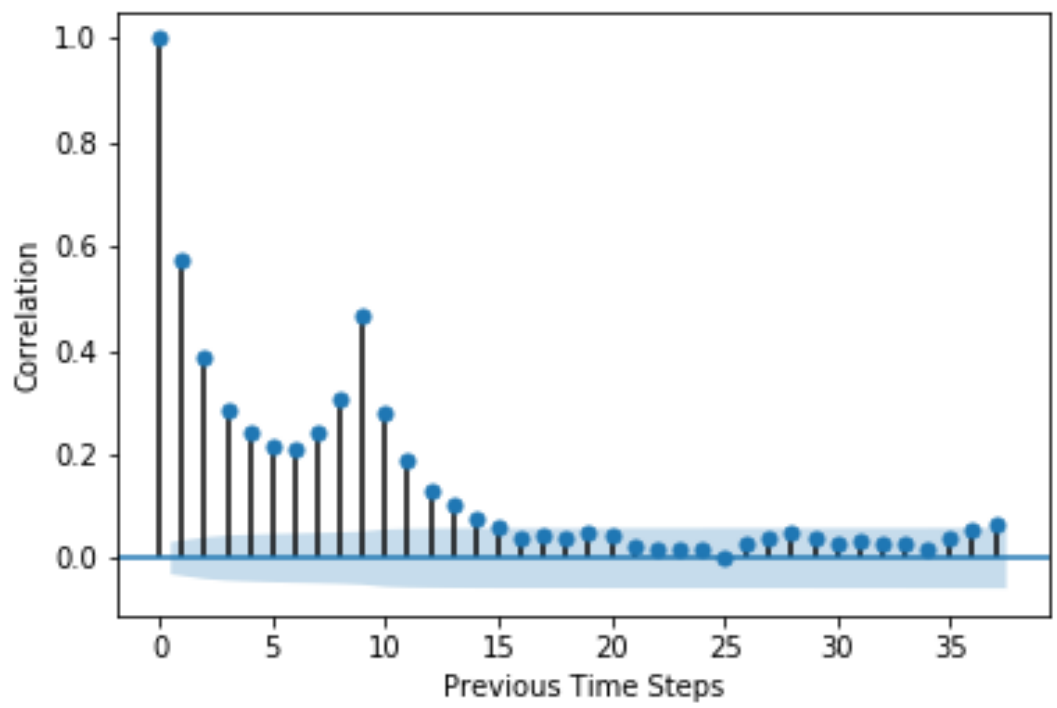

Figure 2.10: NARMA-10 autocorrelation plot showing the time correlations of the sequence, shaded blue area is $95 \%$ confidence intervals of correlation.

This autocorrelation plot shows that when using a linear model for predicting the sequence, there are correlations which could be leveraged to improve prediction performance by including the previous time steps. In the case of NARMA-10 there appears to be significant correlation with the previous 15 time steps. However, correlation can also be considered as a potential for causing error as in linear regression the assumption is that there is no autocorrelation in the inputs. This can lead to increased residual error and steps to adjust for this correlation could improve results. 


\subsubsection{MNIST}

The dataset MNIST consists of a large corpus of handwritten digits from 0-9 [59]. It contains 60,000 training samples and 10,000 test samples encoded as $28 \times$ 28 pixel images. This dataset is used as a baseline comparison for the network's performance on classification due to its being a common benchmark for machine learning classification. While an image may not necessarily be a time series, it can be converted into one either by flattening it out and reading in a single pixel at a time or else feeding in each column or row at each time step. The latter method keeps some of the neighboring features of the image closer together.

An example of the way in which the image was presented to the models can be seen in Fig. 2.11.

The digit image, which is $28 \times 28$ pixels, can be converted into a time-series classification task by converting each row into an input channel and each column into a time step. ESNs have been previously explored for classification accuracy on MNIST [46] where they used a linear regression readout layer. Additionally improvements in accuracy were found by augmenting the data using cropping and rotations.

One of the key aspects for this paper [46] was the exploration of the use of joined state output which contains all of the outputs of the nodes over time vs. last state output which only uses the final output from the reservoir. It is also important to note that it seems the input stream from the MNIST input was also fed into the final readout layer which is a key difference from our research which only uses the reservoirs output for classification.

They explored the lower and upper bounds of 1,000 and 5,000 neurons in the reservoir. They were able to achieve an error rate of $21.2 \%$ for a reservoir of 100 neurons and $9.5 \%$ with a reservoir of 1,200 neurons. Their best error rate of $1.3 \%$ 


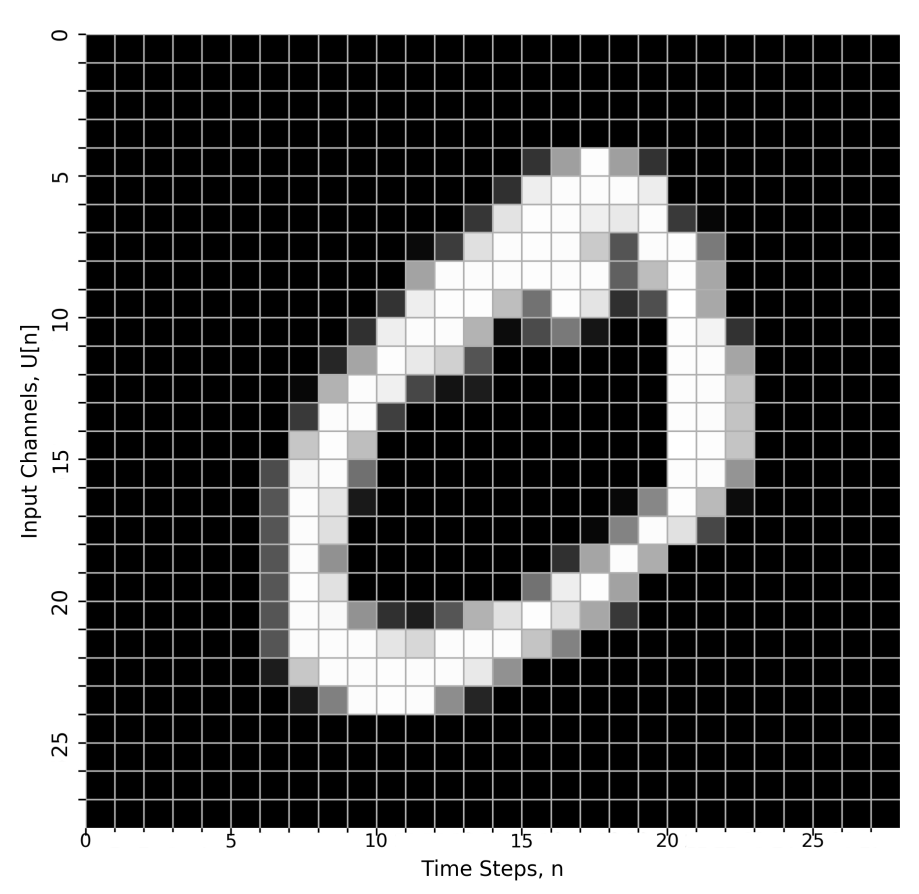

Figure 2.11: Diagram of an example MNIST digit which is $28 \times 28$ pixels. The columns are treated as time steps and the rows are used as input channels, thus converting the task into a multi-variate time series classification task.

was achieved using an ensemble of 21 reservoirs of 1,000 neurons with a joined state output and data augmentations.

Another previous work for MNIST, using reservoir computing, demonstrated using stacked and layered reservoirs [35]. Each layer consisted of 16k neurons and 5 stacked reservoirs which predicted a class at each time step. A final output layer of winner take all for across each of the time steps was used for final classification. Using this methodology they were able to achieve an error rate of $0.8 \%$.

A large ensemble of CNNs has been able to achieve approximately human performance on MNIST of around $0.2 \%$ [60]. 
A chemical implementation of the MNIST task was explored using a winner-takeall readout layer [3]. In this case the MNIST dataset was converted into a binary $10 \times 10$ pixel dataset. A weight matrix was created to represent each class which consisted of the average pixel values for the digits of that class minus the average of the other classes. Also a constraint of only having 20 bits of the 100 bits active for each digit. Based on these constraints only particular digits were selected for the experiments. This was in part due to the need to give the final winner-take-all readout layer a large margin of separation from the other class, the equivalent of around 0.3 for a probability readout layer. In their experiments depending on the classes being differentiated, this resulted in a removal of around $10 \%-16 \%$ of the dataset which would not be experimentally separable.

\subsubsection{Splice Junction Gene Sequences}

This dataset is from the UCI machine learning repository [61], and it is titled the Molecular Biology(Splice-juntion Gene Sequences) Data Set. In a gene sequence there are regions which are removed during RNA transcription, these regions are know as introns. The regions of the gene which will be used in the process to generate mRNA are called exons. The meeting between the two types are called splice junctions. The class of these junctions is determined by their order with intron-exon (IE) junction and exon-intron (EI) junction. The dataset consists of around 3,190 primate nucleotide sequences with a length 60 base pairs (bp) which are labeled as IE, EI, or neither.

Previous results for this dataset was done using a CNN for classification [62]. In this work they used a sliding window to extract the k-mers from the sequences. These 64 possible k-mers were then one-hot encoded and placed together to be analyzed using a 2 layer CNN with max pooling and a final fully connected readout layer of 100 neurons. This paper claimed to have achieved a new benchmark accuracy best 
for this dataset of $96.2 \%$. This is an improvement over the previous best they cite of $94.7 \%$. This accuracy was measured for a 10 -fold cross validation evaluation with the accuracy being reported being the average validation error.

\subsubsection{Dengue Virus Genome}

In this task the aim is to classify the different serotypes of the dengue virus genome based on a 200bp subsample of the beginning 500 nucleotides which contain the structural region of the genome sequence, mainly the untranslated region (UTR) and capsid protein [63]. The dengue virus (DV) is positive strand RNA virus in the family of Flaviviridae. Other popular flaviviruses include zika which is spread by mosquitoes, Aedes aegypti. DV causes dengue fever and infects 390 million people a year, 96 million show clinical symptoms, around 9,000 deaths and and estimated 1.1 million Disability Adjusted Life Years. Symptoms include fever and internal bleeding with a typical recovery period of 2-7 days. Information about DV can be found from the World Health Organization [64]. There are 4 serotypes of Dengue (DENV-1, DENV-2, DENV-3 and DENV-4). People gain immunity from a particular serotype, but can be infected by the different ones and can increase the likelihood of developing severe dengue hemorrhagic fever.

Potentially using chemical reactions to directly detect the virus and serotype could provide useful information to patients and doctors in these poorer rural regions. Some recent work has been done with aptamers for rapid detection of DV using a DNA logic cascade [65].

Some background about viruses, their mutation rates can be significantly higher than that for eukaryotic cells [66]. This leads to a high variability between the different genomes. Within the same serotype genome variation can be 3-5\% and between serotypes 15-20\% difference in terms of amino acids [67] 
The dengue virus genome is 10,000bp. For reference, E. coli have 3 million bp, and humans have about 3 billion bp. [66].

Previous work has been done using k-mer extraction technique followed by an exploration of machine learning classification methodologies [68]. In this study, significantly fewer sequences were used for training, 327, than are used in this dataset which was aquired, 3625. The best performance in the study for accuracy was $96.22 \%$ using neural networks.

Other work for dengue genome classification has been done using k-mer extraction on whole genome sequences [69] which using 10-fold cross-validation was able to achieve $100 \%$ accuracy.

For this research, the dataset was downloaded and compiled using the Virus Pathogen Resource (ViPR) [70] with the search parameters of complete genome sequence, human host, and a label for being classified as either DENV-1, DENV-2, DENV-3, or DENV-4.

An issue which can arise in genomic studies is a disproportionate amount of a particular class over others. This can result in high accuracy from poor decision making on the classifier. For example our dataset of 3,625 DV genomes has a composition of DENV-1: 40\%, DEN-V2: 31\%, DENV-3: 21\%, DENV-4: 8\%. In this case the accuracy can be normalized based on what particular class being classified in order to give a better overall view of the classifiers performance. A confusion matrix is a useful way of characterizing each classes accuracy and can show which classes your model is having the most difficulty differentiating. A simple technique which can be used is either downsampling the more abundant classes or upsampling the smaller classes. Additionally a balanced weighting where the weight for each class is adjusted proportional to its representation can be used for training and evaluation. These methods were explored in this research, although it was found that the class imbalance did not 
necessarily preclude the classifier from performing well.

\subsubsection{Other Dataset Explorations}

\section{Constant Glucose Monitoring}

A task which we helped develop for an undergraduate we are mentoring in the lab was to apply these reservoir computing techniques to a biological dataset for predicting glucose values and hyperglycemia events in diabetic patients. The D1NAMO dataset [71] consists of several days of continuous glucose concentration monitoring of diabetic patients. Previous work has been done on predicting insulin over time using polynomial and auto-regressive models. [72]

A theoretical implementation of this in situ could be a synthetic cell which is able to sense glucose levels and using a CRN determine when to turn insulin production on in the cell. This is theoretical, but could be steps towards an artificial pancreas of sorts.

\section{6s Protein}

Another dataset explored in this research which has been used in work previously when evaluating machine learning for DNA sequences is the 16S rRNA gene. This is used for classifying bacteria as it has a slow rate of mutation and is prevalent in bacteria [55]. The sequences are can be labeled based on their Phylum, Class, Order, and Family. K-mer extraction is the predominant means of analyzing these sequences $[55,73]$ and other work has been done with sequence subsampling and CNNs for classification [74]. 


\section{DeepSEA Dataset}

The DeepSEA dataset consists of non-overlapping 200bp sequences of the ch3 human genome which have been extended on either side to $1000 \mathrm{bp}$. This results in a large dataset size of around 15 million samples. Each of these sequences are labeled with 919 binary outputs for transcription factor activation. Previous work was done which used a CNN layer which fed into a bidirectional LSTM layer and a final fully connected logistic regression layer for binary classification [26]. This model architecture could perform well on many of the genome sequence classification tasks. 


\section{Design Methodology}

\section{Goals}

The design of our experiments is to use an ESN with varying sparsity and size in order to assess accuracy for each of the tasks. These tasks were evaluated using some hyperparameter tuning for the appropriate task. A key aim of this research is to demonstrate that the addition of these reservoirs improves the linear separability of these classes and the prediction capabilities with a small and sparsely connected reservoir. While these ESNs do not necessarily generate state of the art results due to their constraints, that was not the aim of this research.

Our aim was also to explore using different components as the reservoir layer. To bridge the model of echo state networks to chemical reaction networks, a deterministically constructed oscillating neural network, which was based off of the graph of an oscillating chemical reaction network, was used as the hidden reservoir layer. These oscillating neural networks were varied in size and spectral radius and were compared to the performance of using a simulated chemical oscillator as the reservoir layer. A 3 and 4-way chemical oscillator was used as a reservoir layer as well. This work of translating between these defferent non-linear models, aimed to give some insight into the size and level of chemical dynamics necessary for an effective reservoir layer. 


\subsection{Implementation of the Echo State Network Simulations and Readout}

Layer

The reservoir of the echo state network consists of the simple recurrent neural network nodes. These networks were constructed using the PyTorch as well as the Keras framework in Python. The nodes in the ESN consisted of the simple RNNs with tanh activation functions. The initialization technique described for the ESP was performed and the internal weights were set to those fixed values. No gradient was passed to these layers during the training stage as only the output layer was trained.

The Keras framework is a high level abstracted wrapper for Tensorflow and was explored as it is utilized in many of the computational biology papers [75].

PyTorch was incorporated after establishing some proof of concept work with Keras, due to the increased flexibility which is available in the implementation of the neural network as well as its use by others in the lab. Additionally, there was previous work done to implement some reservoir computing using PyTorch known as EchoTorch [76]. This framework seemed to not be up to date with some of the newest versions of PyTorch and so we implemented my own recurrent neural networks for my experiments. We have built guides and tutorials for others in the lab to walk through how to do these experiments using these frameworks.

A key benefit of using these frameworks is the ability to port the model and data to a GPU thus speeding up the computation speed for these networks considerably.

The Python library of scikit-learn was utilized for many of the linear regression , logistic regression, and support vector machine models. The library was also used in steps for k-fold cross validation and grid search of terms in the regularization validation steps.

For training readout layer using gradient descent, settings from previous research 
in biological computing were explored [75]. The optimizer which performed the best and was primarily used was Adam [77], with a learning rate of 0.001 and batch size $=64$. The model was trained until convergence with an early stopping of the model based on when the validation accuracy showed no improvement after 10 epochs. The model with the best performance on the validation accuracy metric was saved.

\subsubsection{Parameter Selection}

In order to optimize performance, before we performed sweeps across the dependent parameters, other hyperparameters were established for experiments. These include the spectral radius, input scaling, inclusion of bias in the reservoir, learning rate, and batch sizes. On the tasks for ESN where the sparsity and number of nodes, $N$, is varied, an initial parameter sweep for an ESN with $N=100$ was done along the parameters of input scaling, spectral radius, bias, activation functions, and dropout for regularization. The input scaling was varied for $U[-a, a]$ from $a=[0.1-1.0]$ in 0.1 increments. The spectral radius search was set as 0.9 as a default and explored for spectral radius $=[0.1,0.3,0.5,0.7,0.8,0.9,0.95,0.99]$ for the different classification tasks. A value of spectral radius $=0.9$ was found to perform well on the tasks and was fixed for the sparsity and size explorations. The different internal activation functions of sigmoid, tanh, and ReLU were explored with tanh performing the best and was used in all of the ESNs and oscillating neural networks. Interesting to note is that the tanh has the ability to output negative values.

In order to characterize how some of these parameters affect the dynamics of the reservoir an ESN with $N=100$ was constructed and fed a $\delta[n]$ impulse as input, which is a 1 at $n=0$, and 0 for all other $n$. The impulse response of the network was monitored in terms of the sum of the node outputs and the sum of the absolute values of the node outputs. 


\section{Spectral Radius}

The spectral radius is a parameter which affects the fading memory in the network and a value of $<1$ is used in order to maintain the echo state property [36]. This makes it such that the reservoir states eventually are unaffected by initial inputs. If the spectral radius is larger than 1, there is not as rigorous of a proof that this fading memory occurs for all instances. In practice, this requirement of spectral radius $<1$ has been shown to not always be necessary [46] and in some cases the performance on a particular task can be improved by increasing the spectral radius to larger than 1 although an additional "leaky" term is sometimes added which causes the reservoir outputs to decrease over time. These effects for spectral radius $<1$ and $>1$ on behavior of the reservoir are demonstrated in Fig. 3.1 and Fig. 3.2.

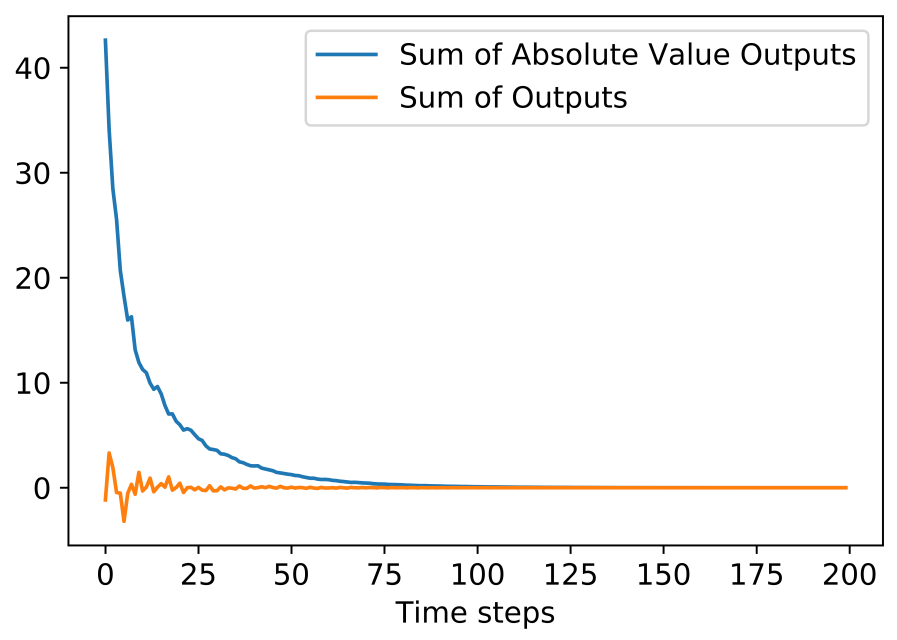

Figure 3.1: Node outputs after a $\delta[n]$ input to an ESN with $N=100$, spectral radius $=0.95$, and no bias. The network experiences a decay over time due to the spectral radius being $<1$. 


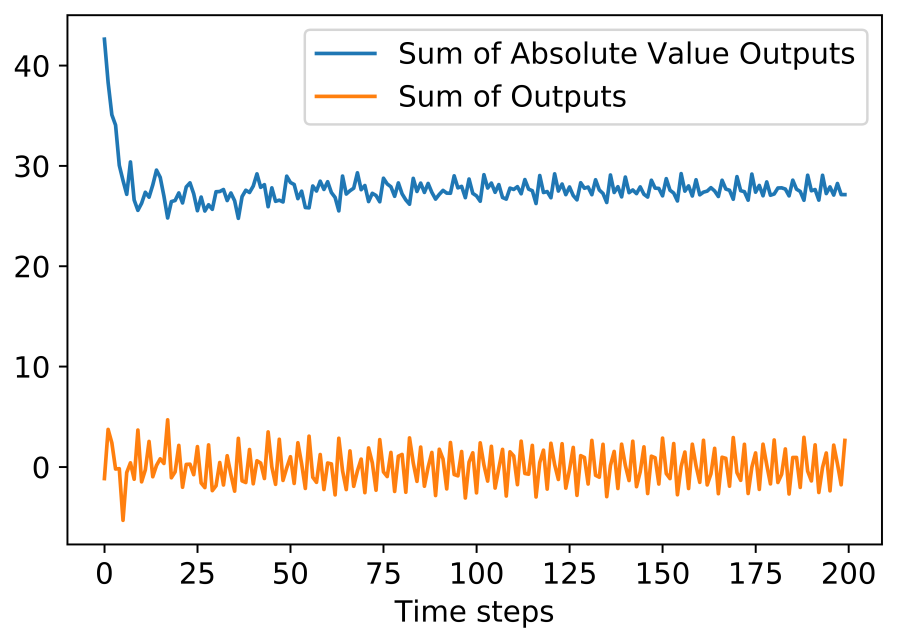

Figure 3.2: Node outputs after a $\delta[n]$ input to an ESN with $N=100$, spectral radius $=1.1$, and no bias. Note how the network node outputs do not decay after the initial input and continue to output values which fails at achieving the desired echo state property as past inputs will always affect the future state of the network.

\section{Sparsity}

The sparsity of the ESNs were varied in order to attempt and reduce the complexity of these networks and thus make them more applicable and feasible for potential physical implementation. Some previous work has been done on reservoirs with scalefree properties and other topologies such as small world networks [16]. However it was found that many of these topologies performed about as well as the random fully connected networks.

It can be noted however that there are different dynamics observed from sparse networks as they seem to form localized networks which can cause dynamic oscillations in the network. This is demonstrated in Fig. 3.3 where, for a sparse network, the node outputs are decaying over time, however they show oscillations due to the addition of sparsity which may be causing local networks to form [78]. 


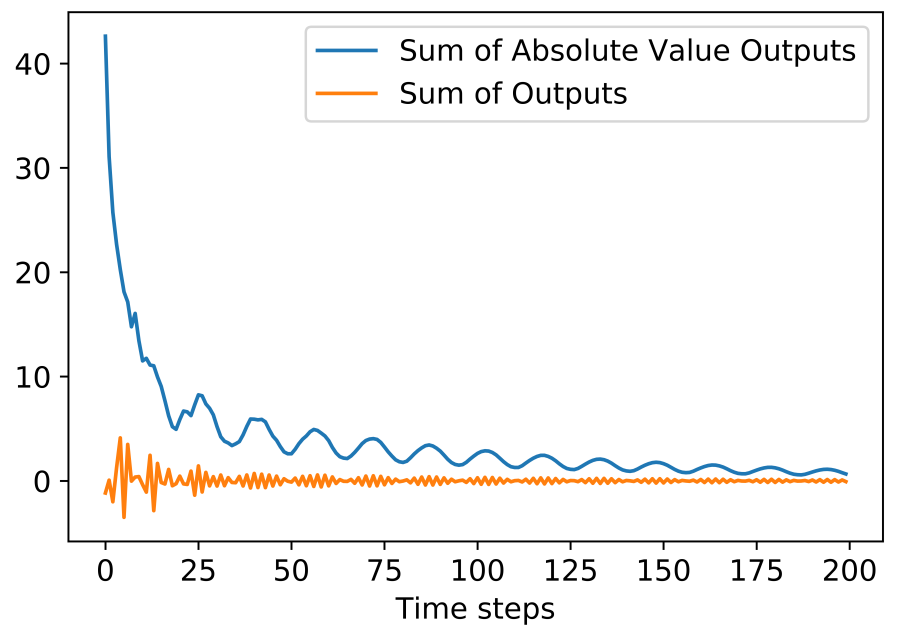

Figure 3.3: Node outputs after $\delta[n]$ input to an ESN with no bias, $N=100$, spectral radius $=0.95$, and sparsity $=0.1$. Note the oscillatory behaviour of the network nodes' output due to the addition of sparsity which differs from that in Fig. 3.1.

When implementing the sparse networks, the sparsity was treated as a probability of a connection forming between nodes which can cause high variance for small networks. For smaller networks a more constrained sparsity definition can be used where the proportion of connections is less than or equal to the sparsity.

\section{Bias}

In order to ensure the reservoir in the ESNs contain the desired ESP, whether or not to add bias to the reservoir connection matrix, $W_{\text {res }}$, was explored. These explorations into the impact of having a uniform bias initialization showed there was not a significant change in accuracy and, due to the desire for the reservoir to return to a zero state of equilibrium if no input is passed into the reservoir according to the ESP, the bias weights for the reservoir on all the tasks was set to zero.

With bias the reservoir will settle to some state of equilibrium or may be unstable in some cases where oscillatory behavior is present. Additionally if the hidden states 
are initialized to zero, the presence of bias will cause the nodes to output values when no input has been given.

The bias is present in the readout layer and the weights are trained which allows the final logistic regression layer to be more effective. In the case of a linear regression to the output the bias is important as it makes the best fit line affine rather than linear, the difference between $y=W x$ and $y=W x+b$. This output bias weight is able to be trained using backpropagation and the Moore-Penrose equations.

To show the impact of adding a reservoir bias on the overall reservoir, the activity is shown in an ESN with $N=100$ and tanh activation, which has been fed a $\delta[n]$ impulse, and then its activity has been plotted in Fig. 3.4.

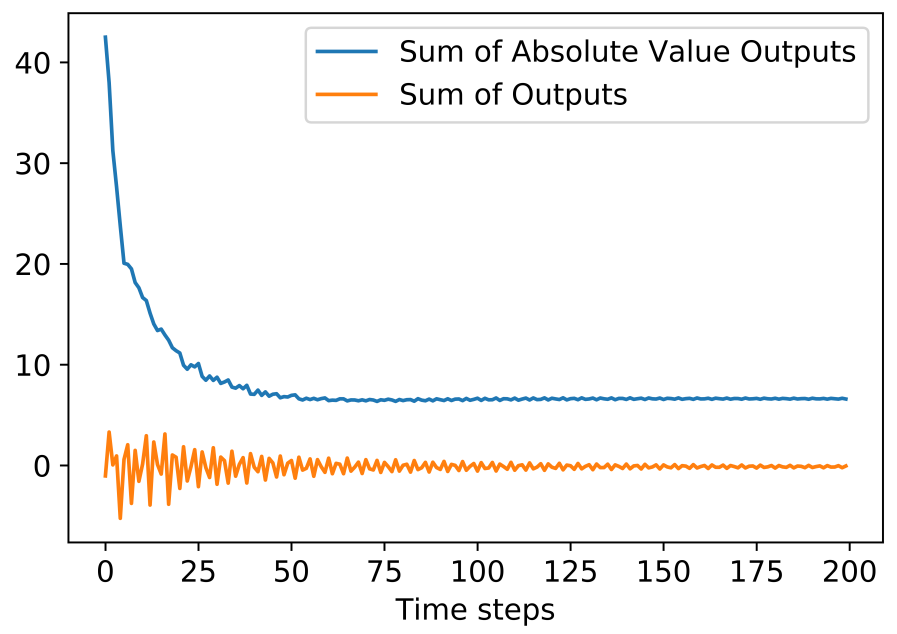

Figure 3.4: Node outputs after $\delta[n]$ input to an ESN with $U[-0.1,0.1]$ bias, $N=100$, spectral radius $=0.9$, and sparsity $=1.0$. Note difference in equilibrium state which differs from that in Fig. 3.1 and is more similare to the network without the desired ESP in Fig. 3.2. Due to this difference in output, the bias for the reservoirs of ESNs was set to 0 .

In this case a positive weight for the bias shifts the node's output to the positive side, and a negative weight shifts the node's output to be in the negative value at equilibrium which results in a sum output of zero but a higher absolute value of 
output.

\subsubsection{Oscillating Neural Network}

In reservoir computing the hidden layer can be treated like a black box which can be replaced by some form of complex dynamical system which is a non-ESN style reservoir [16]. Previous work has been done using chemical reaction networks which were oscillatory in nature for the task of reservoir computing [?,7].

We experimented with a deterministically constructed oscillating neural network for the reservoir layer. This is similar to a constructed cycle reservoir which was shown to be able to have a memory of size $N$ [50], but with some additional recurrent and negative weights.

The matrix of the oscillator was constructed based on the layout of the recurrent oscillator [10] shown in Fig. 3.6.

Positive and negative weights are analogous to activating and inhibitory chemical reactions. The self-recurrent nature of the network also allows for autocatalytic reactions. From this diagram an adjacency matrix for a recurrent neural network was constructed.

This results in a weighted connection matrix:

$$
\left[\begin{array}{cccccc}
1 & 1 & 0 & 0 & 0 & 0 \\
0 & 0 & -1 & 0 & 0 & 0 \\
0 & 0 & 1 & 1 & 0 & 0 \\
0 & 0 & 0 & 0 & -1 & 0 \\
0 & 0 & 0 & 0 & 1 & 1 \\
-1 & 0 & 0 & 0 & 0 & 0
\end{array}\right]
$$




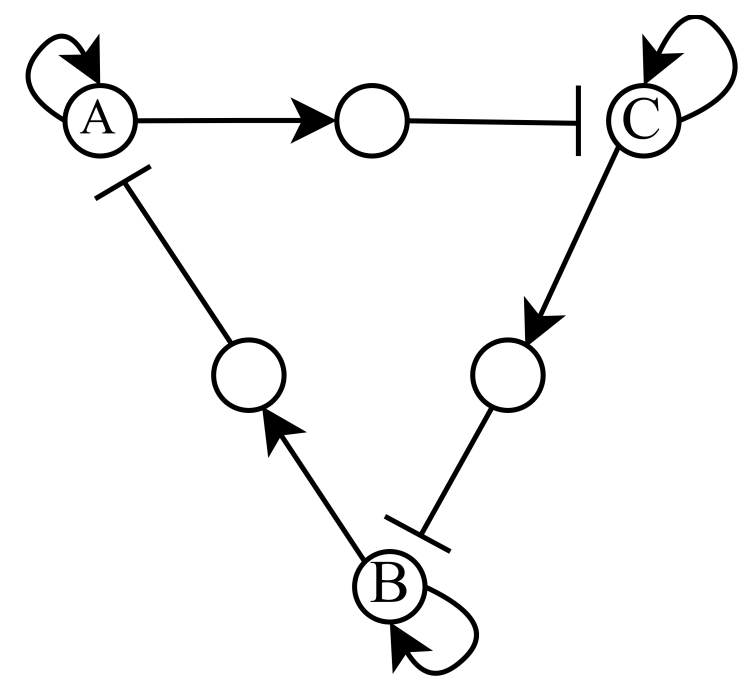

Figure 3.5: Structure of the deterministically constructed oscillating neural network. This construction was based off of the DNA inhibitory oscillator described $[7,10]$, where the inhibitory chemical reaction connections are mapped to negative weights and the activating chemical reaction connections are positive weights. The network can be extended to have more nodes and become a large oscillating cycle. The arrows indicate positive/activating connections and the bars indicate negative/inhibitory connections.

The spectral radius of this network was scaled to be $<1$ which achieves the desired ESP. The use of this oscillator aims to demonstrate a reservoir layer can still be effective when constructed similarly to a chemical oscillator graph structure and may allow for an easier or more justified conversion between the two providing a stepping stone towards implementation in chemistry. Future work could be to attempt to map the minimum complexity ESNs to a chemical reaction network.

The oscillating and decaying behavior of this oscillating neural network can be seen in Fig. 3.6.

The oscillating neural network was explored based on the deterministic construction which was then scaled have different spectral radius values $=[0.1,0.3,0.5,0.7$, $0.9,0.95,0.99]$. These spectral radii were also considered for different sizes of network for $\mathrm{N}=[6,10,14,30,42,62,102,162,322,502,1002]$. The values for $N / 2$ need to 


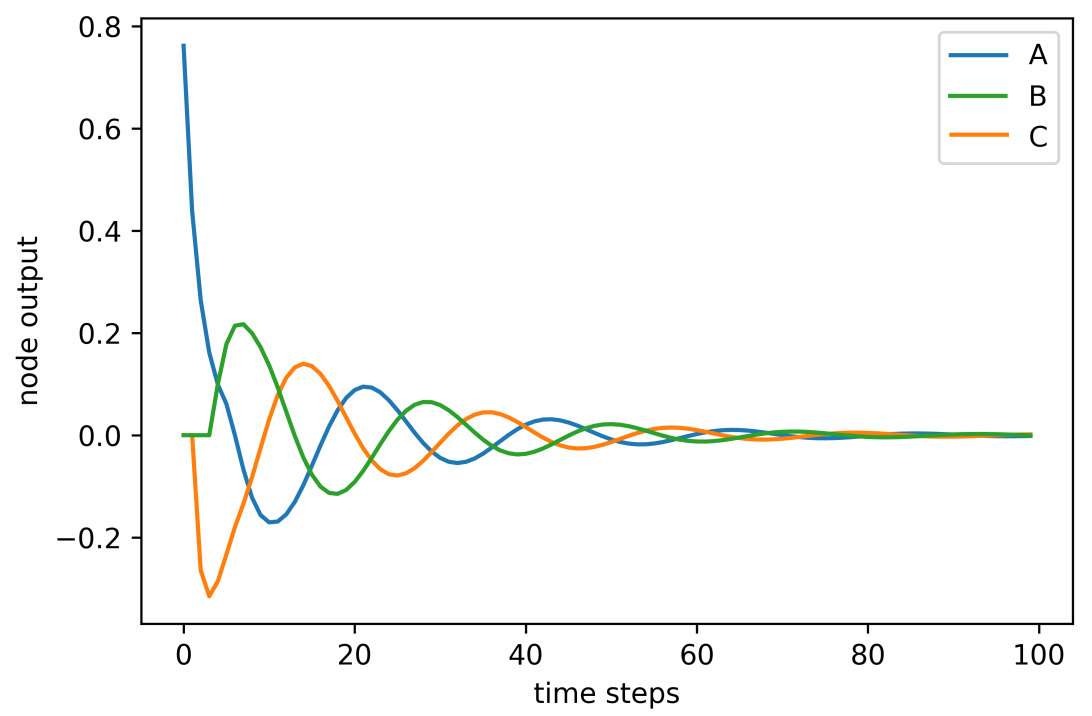

Figure 3.6: Node outputs from the deterministically constructed oscillating neural network when fed the $\delta[n]$ input. The network was scaled to have a spectral radius= 0.9 and demonstrates an oscillatory behavior centered around a mean output $=0$ with a gradual decay.

be odd in order for the network to function which is why some odd values like 1,002 instead of 1,000 were selected.

Based on the initial experiments with the oscillating neural network, it was found that the inputs should be scaled to incorporate some level of non-linearity into its model. Since the oscillator reservoir was using tanh activation functions it can be noted in Fig. 2.3 that for the output values of -0.5 to 0.5 , the tanh function is approximately linear. When the oscillatory network was adjusted to only operate in this regime, There was not a significant increase in classification accuracy achieved. However, if the values of the oscillator were shifted to be in the non-linear regime, there was an increase in the classification accuracy.

An example of using the oscillating neural network as a reservoir and its similarity in dynamics to the chemical oscillator is shown in Fig. 3.7 and can be compared with 
the outputs from the chemical oscillator which was fed the same input in Fig. 3.8.

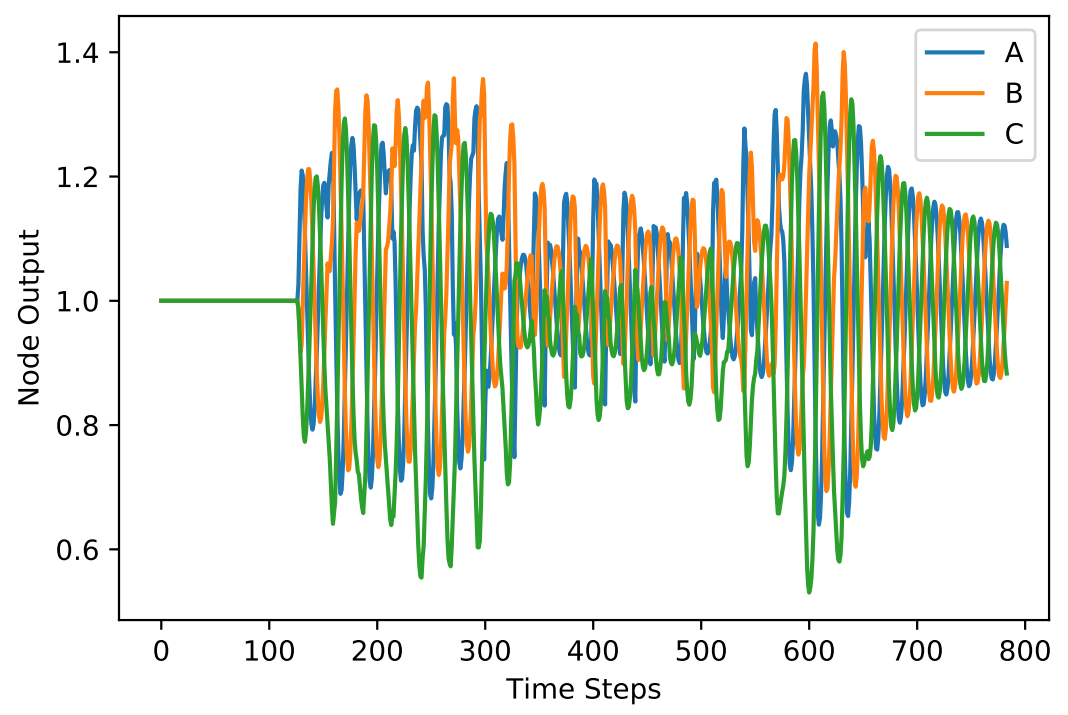

Figure 3.7: Example of the neural oscillator as the reservoir layer with an MNIST digit input, a 0, being fed in one pixel at each time step as a scaled value of $A$ and the outputs have been shifted to have an equilibrium of 1 and normalized in the same way as in Fig. 3.8.

While there are different non-linearity between the neural network and chemical oscillators, they may qualitatively work well as a reservoir layer with somewhat similar projections.

\subsubsection{Chemical Oscillator as a Reservoir}

The concept of reservoir computing can be extended to using, for the reservoir hidden layer, a simulated chemical oscillator. Here we explore the 3-way chemical oscillator model previously mentioned, the displacillator [9].

An example of how this reservoir step performs as a reservoir is shown in Fig. 3.8 where an MNIST digit, 0 , is flattened into a $784 \times 1$ input stream which is fed into the chemical oscillator as a scaled concentration of chemical $A$. Each pixel is treated 
as a time step and the initial concentrations and reaction rates are set to 1.

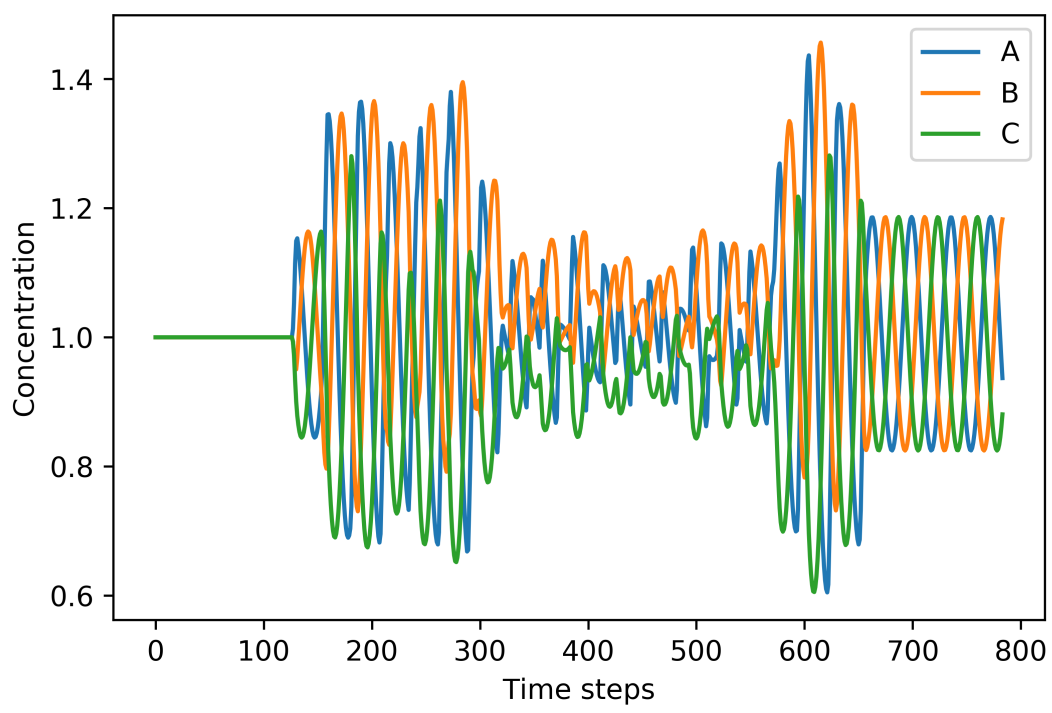

Figure 3.8: Example of the chemical oscillator acting as a reservoir layer with an MNIST digit input, a 0, being fed in one pixel at each time step as a scaled concentration of the chemical $A$. These chemical oscillations can then be used by a readout layer to make the classification.

In previous work [3], they only experimented with around 100 digits also due to the difficulty of simulation.

For the genome sequences a modified 4-chemical oscillator was generated with the this was done in order to map each of the nucleotides to a particular chemical which might make it easier.

\subsubsection{Encoding DNA Sequences}

A typical DNA sequence consists of a string of nucleotides, ATCG. In order to process the data and train with it these need to be encoded numerically. There are several ways to encode such as ordinal, binary, and one-hot.

Here we present some examples of how this would work. 
Sequence $=G A T T A C A$

Ordinal: $A=1, T=2, C=3, G=4$, Sequence $=[4,1,2,2,1,3,1]$

Binary: $A=00, T=01, C=10, G=11$, Sequence $=[11000101001000]$

One-hot: $A=[1,0,0,0], T=[0,1,0,0], C=[0,0,1,0], G=[0,0,0,1]$

Sequence $=\left[\begin{array}{cccc}0 & 0 & 0 & 1 \\ 1 & 0 & 0 & 0 \\ 0 & 1 & 0 & 0 \\ 0 & 1 & 0 & 0 \\ 1 & 0 & 0 & 0 \\ 0 & 0 & 1 & 0 \\ 1 & 0 & 0 & 0\end{array}\right]$

While the one-hot encoding adds a dimension to the data, it more accurately represents the difference between the nucleotide inputs.

There are also some other base pairs other than ATCG which are encoded as proportional values according to their possibilities, e.g., an $\mathrm{S}$ is either a $\mathrm{T}$ or $\mathrm{C}$ so it is encoded as $[0,0.5,0.5,0]$.

As input into the network, a genome will be one-hot encoded and then fed in one base pair at a time, therefore one base pair can be modeled as one time step essentially treating the genome as a multivariate time sequence classification problem with an input length the length of the genome, $L$.

One-hot encoding generates an $L \times 4$ input sequence to the reservoir where each index along $L$ is treated as a time step for input, $U[n]$. Each of these four array values at each base pair index are then fed in as input to the reservoir. The inputs are weighted according to the $W_{i n}$ matrix which in this case is $4 \times N$ matrix, where $N$ is the number of nodes in the reservoir. A diagram showing how

Categorical data is typically one-hot encoded. This is effective when the distance 


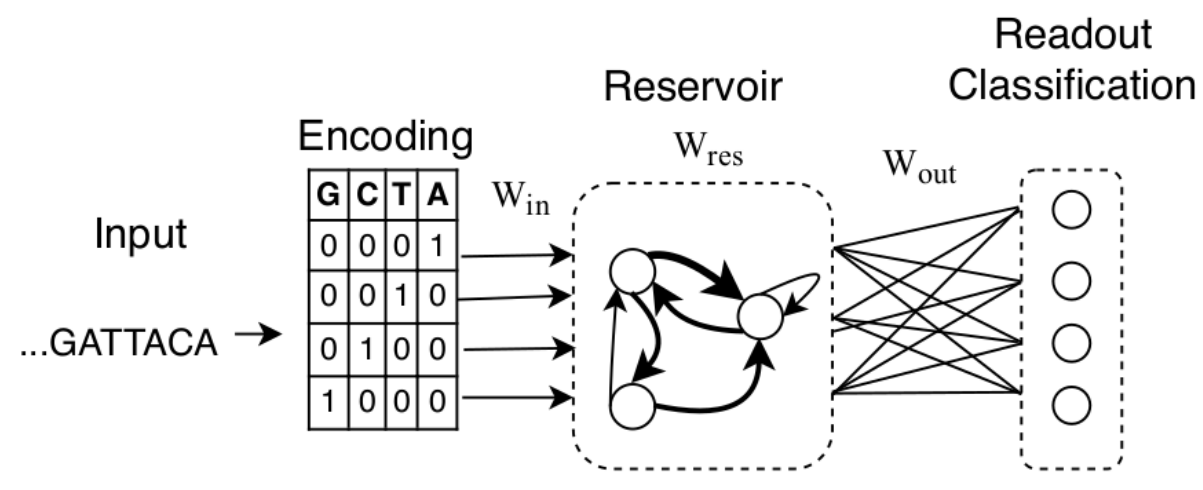

Figure 3.9: Layout of the reservoir system with the input being a one-hot encoded genome sequence. The readout layer in this case is designed to classify the input sequences based on the states of the reservoir over time as the genome is fed in.

metric between the different parameters is the same between each of the categories. In this case the difference between and $\mathrm{A}$ and $\mathrm{T}$ is the same as the difference between an $\mathrm{A}$ and $\mathrm{G}$, which is a good first approximation.

Some exploration was done into encoding the inputs into the network as k-mers which were then one-hot encoded as well. So for $k=3$, there will be $k^{4}$ encoding possibilities. This k-mer encoding could be more feasible for physical implementation and improve performance.

It may be possible in the future to develop a more a posteriori information theory about these nucleotide and k-mer distributions and thus embed them in a more representative vector such as with natural language processing $[79,80]$.

\subsubsection{Training and Validation}

For the purpose of exploration a dataset can be split into approximately $80 \%$ training set and $20 \%$ validation set. There can also be a test set which is held out in order to improve generalization of the performance. The validation set is used for the early stopping and cross-validation on the training set is used for initial tuning of hyperparameters of the model before doing the sweeps. 
For the MNIST dataset, the 60,000 training samples are used to train the models and final reported accuracies are performed on the 10,000 test samples. The reservoir parameter sweeps are done using this setup with three different random seed values.

For $\mathrm{k}$-folds validation, the dataset is split into $k$ sections or folds. A single fold is held out for validation and the model is then trained on the rest of the folds. This is repeated for all of the folds and the final validation value is the average. This can be useful for hyperparameter tuning and utilizing the full benefit of the size of the available dataset. This was done on the Splice Junction Gene Sequences dataset due to the small size, 3k, and due to this being the reported value in literature which used 10-fold validation.

For the dengue virus, the subsampling was used to generate a larger dataset size from the 3,625 original genomes 54,375 subsequences were extracted and split into the $80 \%$ training and $20 \%$ validation sets.

\subsection{Physical Implementation}

Here we present some initial collaboration between myself and a PhD student at University of Mexico on how one might implement these networks in physical chemistry. His work in biochemistry and nanotechnology techniques provided some insights and references as to what steps would need to be taken to potentially build some realization of these concepts in chemistry.

\subsubsection{Serotype Detection Assay}

We propose the use of DNA logic in the context of a reservoir network based classifier that will use the direct genomic data sensed to classify serotype. A classifier scheme may take advantage of the existence of abundant data sources and use much of the 
available nucleotide in a sample. A DNA logic implementation of a classifier has been used to successfully classify bacteria from virus based on expression profiling [2]. Inspired by this work, we seek to expand upon the classifier idea by using a reservoir. Instead of transcription products, viral genomes will be used as the input to a classifier. Viral genomes are similar to an expression product given the paradigm of their manufacture.

The foundation of the physical implementation of the classifier assay is the Unified Sensor Architecture (a fusion of TMSD with DNAzyme elements and FRET reporters) [81]. This architecture will execute the biosensor function with a feed-in to the signal into the classifier which will be implemented in DNA [82].

\subsubsection{Determining Biochemical Constraints of the System}

Implementing this system would require designing oligonucleotides that interact with each other in a manner that recapitulates the reservoir function $[7,82]$. This requires design based on thermodynamic constraints, so NUPACK [83] software will be used. The resulting design is based on thermodynamic equilibrium, strands will need to be tested to ensure no kinetic traps. Based on wet-lab results, chemical kinetic modeling will be done to determine rate constant parameters. Rate parameters and concentration are the key systemic constraints. The microfluidic system has variables that contribute to the systems performance: volume and pump speed. Concentration of components will be maintained by the small size of the microfluidic device. Pump speed will determine inflow of inputs and outflow of outputs. The nanoparticles used for the translator will be a potentially complex additions to the system, but have the advantage that they will be more stable than the solution-based components and can maintain a physical permanence.

The potential physical implementation of this classifier has been proposed above, 
but what are the prospects for using DNA to embody the classifier as designed? For the classifier to be clinically useful, the ideal input into the system should be the unmodified genome of interest. The genome sequence must then be converted into oligonucleotides that are capable of interacting with downstream elements of the classifier. Sequence information on the genome comprises all of the individual bases and the order in which they appear. Individual bases may be converted through a chemical converter as previously mentioned [84], but the sequence information may be lost. The classifier treats each base as a time step, so it is preferred that a read of the genome proceed in order (ideally 5' to $3^{\prime}$ ). In solution, molecules will interact randomly, so there is no guarantee of transfer of sequence order. This may be mitigated by splitting the genome into partitions of smaller size and then assaying each partition separately. Size exclusion may be used to separate each of the partitions. As a microfluidic approach makes sense for this circuit [82], the control in separation may be achieved so. Smaller sizes can work against accuracy so this design consideration is working against optimization of partition size and accuracy. 


\section{Results}

In this section, we will present the results of our experiments using the ESNs with an aim to identify where the addition of the reservoir layer improves performance over a baseline memoryless linear model and a linear model with memory of the inputs. The classification linear baselines will use a flattened input with logistic regression for comparison.

The oscillating neural networks and the chemical oscillator are also used as a reservoir layer and their performances are reported here with discussion of their implication and usefulness. We also discuss trends in the data with regards to the parameters being plotted of size, sparsity, and spectral radius.

\subsection{Sequence Prediction}

\section{NARMA}

The non-linear autoregressive moving average (NARMA) task was explored as it is a common benchmark used in the reservoir computing literature, has time dependency on inputs and previous outputs, and can be generated using the equations mentioned in the background section. For the ESN, these results do a sweep across the different sparsity and reservoir size parameters with a spectral radius $=0.9$. The aim of this was to demonstrate that these ESNs were capable of learning a memory of the input and then combining them according to the desired equation with minimal size and sparsity.

For each of the NARMA series, a sequence of 4,200 was generated, the first 200 
values were removed due to initial transience and the next 2,000 steps were used for training and the final 2000 steps were used for testing. The NARMA series were generated 5 times using different random vector inputs and 5 ESNs of each parameter were tested for each series.

The average MSE is reported for each task and the standard deviation error values are contained in the Appendix tables.

\subsubsection{NARMA Task A}

This task only requires a memory of the previous 2 inputs and a linear combination of those inputs in order to effectively generate the desired output. This task should be easier than the other tasks due to this straightforward construction. A linear model which based on only the current input value, memoryless, achieves an MSE $=0.42$. A linear model with a memory of size 2 achieves $\mathrm{MSE}=0.0$ which is exactly what is expected.

\subsubsection{Echo State Network}

After the random vector is passed through the ESN, the output states are used to predict the output sequence. Using spectral radius $=0.9$ and with input weights $U[-0.1 .0 .1]$, the results in Fig. 4.1 were achieved using ridge regression with the $L 2$ regularization term being optimized for each network using k-fold cross-validation on the training sequence, $k=5$.

\section{Discussion}

The NARMA-A task can be solved using a memory of the previous 2 inputs and the ability to linearly combine these values to match the target output. In order to perform better than a linear memoryless model the minimum complexity of the 


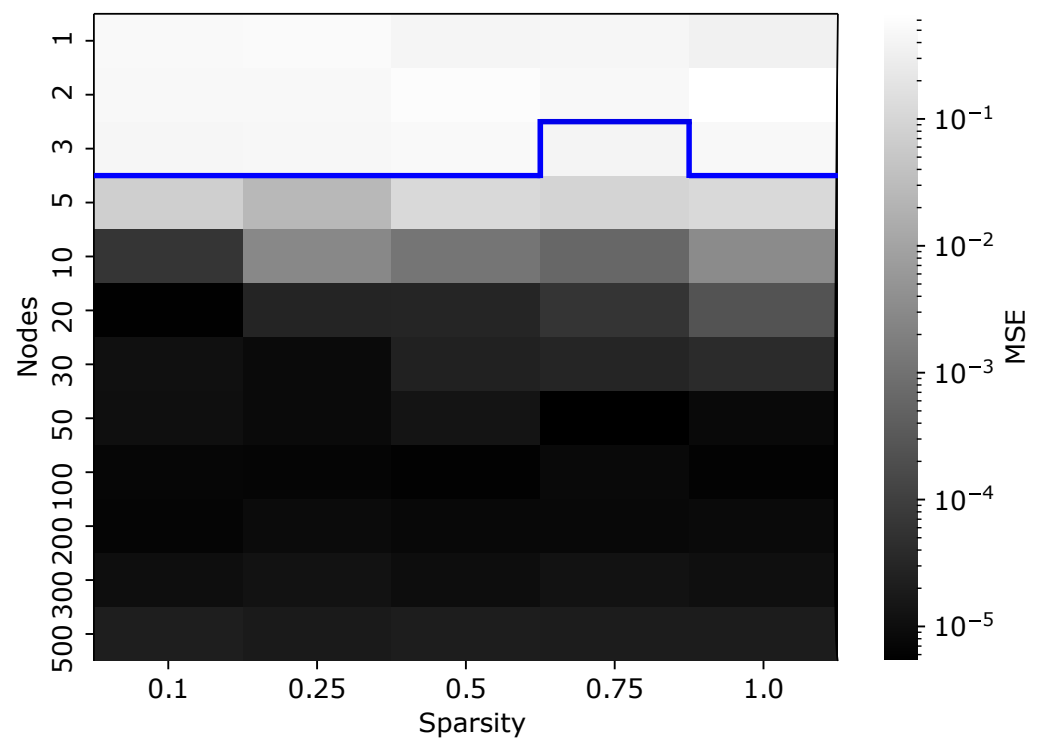

Figure 4.1: MSE of the ESN on NARMA-A. The blue lines represents the threshold for the baseline memoryless linear regression, $\mathrm{MSE}=0.42$. The network improves this baseline with a minimumum complexity of $N=3$, sparsity $=0.75$ and improves with larger networks plateauing in performance at $N=20$, sparsity $=0.1$ with an MSE $=5.6 \times 10^{-6}$

ESN was $N=3$ with a sparsity $=0.75$. Here we give preference to the smaller node value even though there may be fewer total connections in other networks say of size $N=5$ with sparsity $=0.1$. The ESNs are able to achieve a low MSE error of $5.6 \times 10^{-6}$ at $N=20$ with sparsity $=0.1$, however it struggled to achieve a lower MSE as the network grew in size. One issue may have been the spectral radius was set too high for this ESN at 0.9 which will be explored using the neural oscillating network. Additionally at these smaller error values, converging to a solution can be more difficult as the gradient of the error is also proportionally small. 


\subsubsection{Oscillator Networks}

We next experimented on the NARMA-A task using the oscillating neural network described. Input weights were $U[-0.1,0.1]$ and the results for a sweep of this network across size and spectral radius is shown in Fig. 4.2.

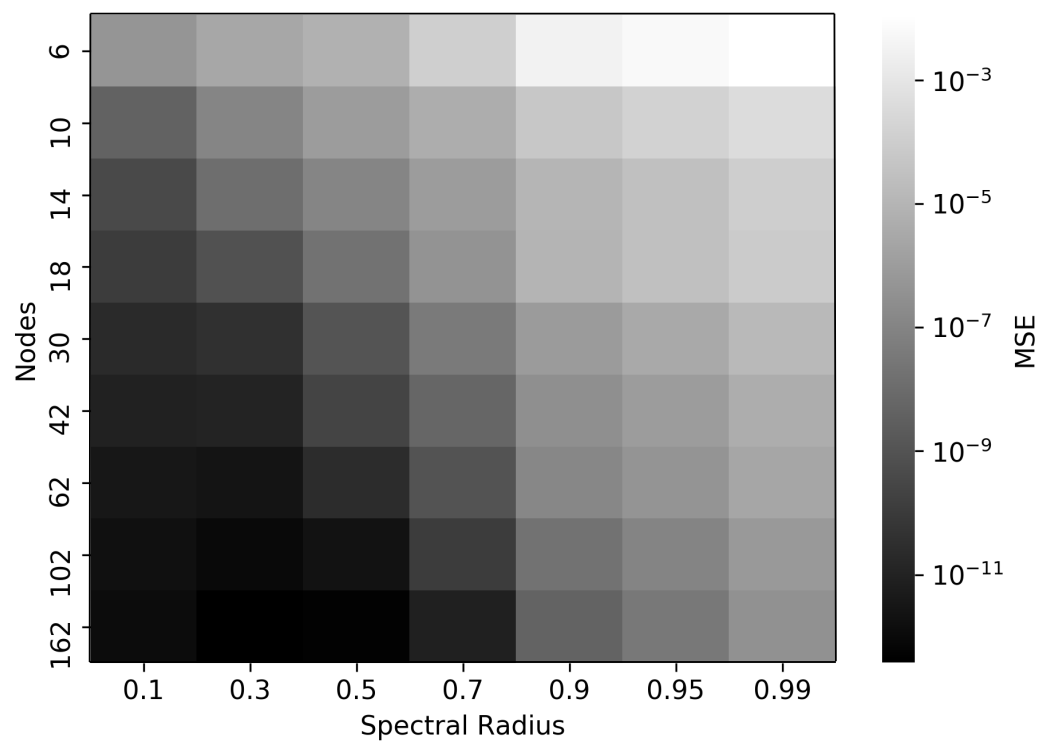

Figure 4.2: MSE of the oscillating neural network on NARMA-A across different spectral radius and reservoir sizes. All of the settings performed better than the linear memoryless baseline of $\mathrm{MSE}=0.42$. The network was able to converge to a very small $\mathrm{MSE}=3.8 \times 10^{-14}$ using $N=162$ and spectral radius $=0.3$. To note is that a linear model with a memory of size 2 can perfectly solve this task.

The 3-way chemical oscillator network was also used for this task with the input being fed into the network as a scaled input of chemical A. An input scale of 0.5 was used and an an MSE $=0.33$ was achieved which is slightly better than the memoryless linear model. 


\section{Discussion}

Interestingly the oscillating neural network was able to accomplish very high accuracy with a larger size and smaller spectral radius. This is could be due to the very low memory requirement of this task and thus having a small spectral radius which makes the network have a quicker fading memory is beneficial for this task. The chemical oscillator was able to only slightly improve over a memoryless linear model. There may be ways of improving this by incorporating the memory of the oscillator into the predictions or adjusting the input weights. Other variables to adjust could be to measure the oscillator with a delay after input which could allow the chemical dynamics more time to interact and thus improve performance.

\subsubsection{NARMA-10}

The NARMA-10 task is more difficult than NAMRA-A as it requires a larger memory in order to solve the task due to the longer term dependencies which are shown in the equation for NARMA-10 as well as the non-linear combination of the input sequence. Using a memoryless linear model achieves $\mathrm{MSE}=0.0088$ and a linear model with memory achieves $\mathrm{MSE}=0.0018$.

\subsubsection{Echo State Networks}

The random vector input was passed through the ESN and the readout layer was trained to match the target output. Using spectral radius $=0.9$ and with input

weights $U[-0.1 .0 .1]$, the results for the performance of the ESNs on the NARMA-10 task can be seen in Fig. 4.3 


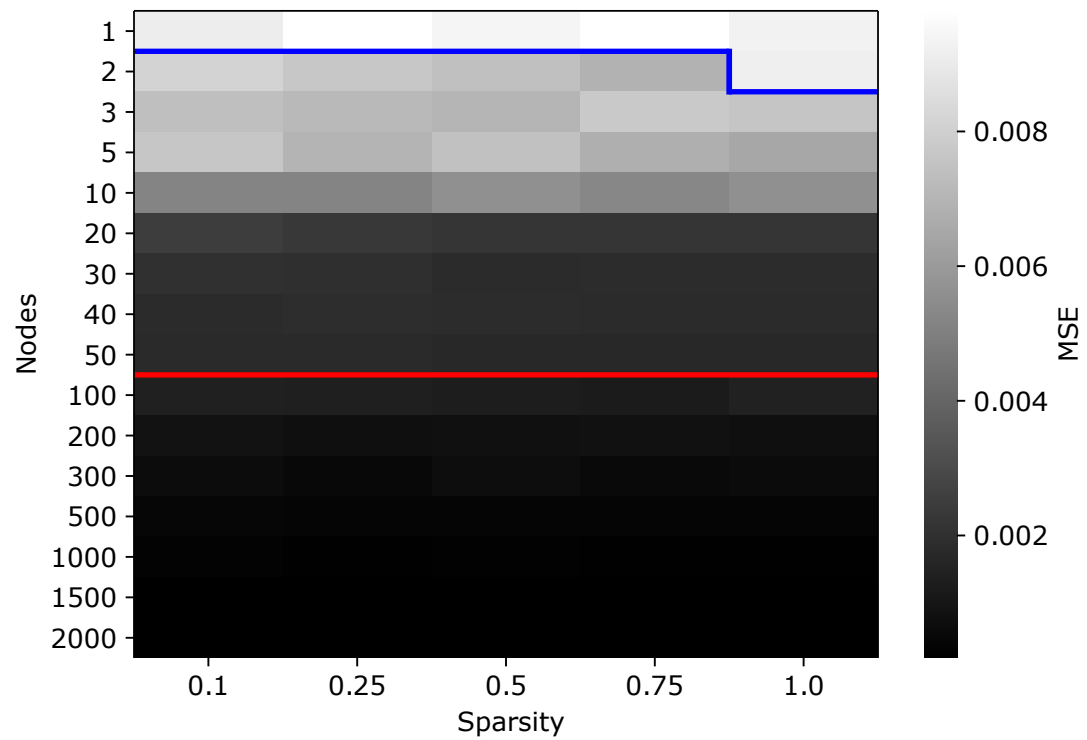

Figure 4.3: MSE performance of the ESN on the NARMA-10 sequence across different $N$ and sparsity values, the blue line represents the linear model without memory and the redline is a linear model with input memory. With only 2 nodes the network is able to capture input memory and combination and at $\mathrm{N}=100$ the ESN is able to achieve better than the linear regression model with input memory even with low sparsity.

\section{Discussion}

The NARMA-10 results show that the ESN is able to store some form of memory within the node values. This causes an improvement in the MSE measured for the reservoir as the number of nodes are increased. The network is able to achieve better than memoryless linear models with only $N=2$ and small sparsity. In this case sparsity is used as the probability of a connection forming between nodes in the reservoir. This can cause high variability for the smaller networks and perhaps a more stringent constraint for connections in the reservoir to always be less than the value could reduce this variation in performance.

The ESN was able, with $N=100$, to achieve higher than linear models with 
memory of the input. These linear models were explored with different memory sizes and they reached best performance with memory size $=50$ and no improvements with larger memory size. Initial explorations show the sparsity of the network does not affect a significant change in performance from sparse to fully connected networks at the larger $N$ values. This is informative in terms of design for future networks as it shows that a network with significantly fewer connections is still able to maintain the necessary memory and computation in its nodes in order to perform well on the task. There is a significant improvement in performance of the model as the number of nodes in the reservoir are increased up until $N=1500$ after which gains are insignificant.

Also to note is that this ESN implementation produced results similar to previous reported values [58] with the network achieving similar MSE results for the same settings. In [58] an MSE $=3.14 \times 10^{-4}$ was achieved for $N=500$, and $1.80 \times 10^{-3}$ for $N=100$. We found $\mathrm{MSE}=3.8 \times 10^{-} 4$ for $N=500$, and $1.4 \times 10^{-3}$ for $N=100$. These comparisons were done on a fully connected network with spectral radius $=0.9$. The variation could be due to different input initialization and network variability from the random connections. When accounting for the margin of errors there is only a small variation.

\subsubsection{Oscillator Networks}

For the NARMA-10 task the oscillating neural network was explored with its size and spectral radius being varied. Results for the NARMA-10 problem using the oscillating neural network can be seen in Fig. 4.4.

This task was also performed on the 3-way chemical oscillator with the input being fed in as a concentration of the chemical $A$ with an input scaling of 0.8 . This achieved an $\mathrm{MSE}=0.0066$. This is better than the memoryless linear model and 


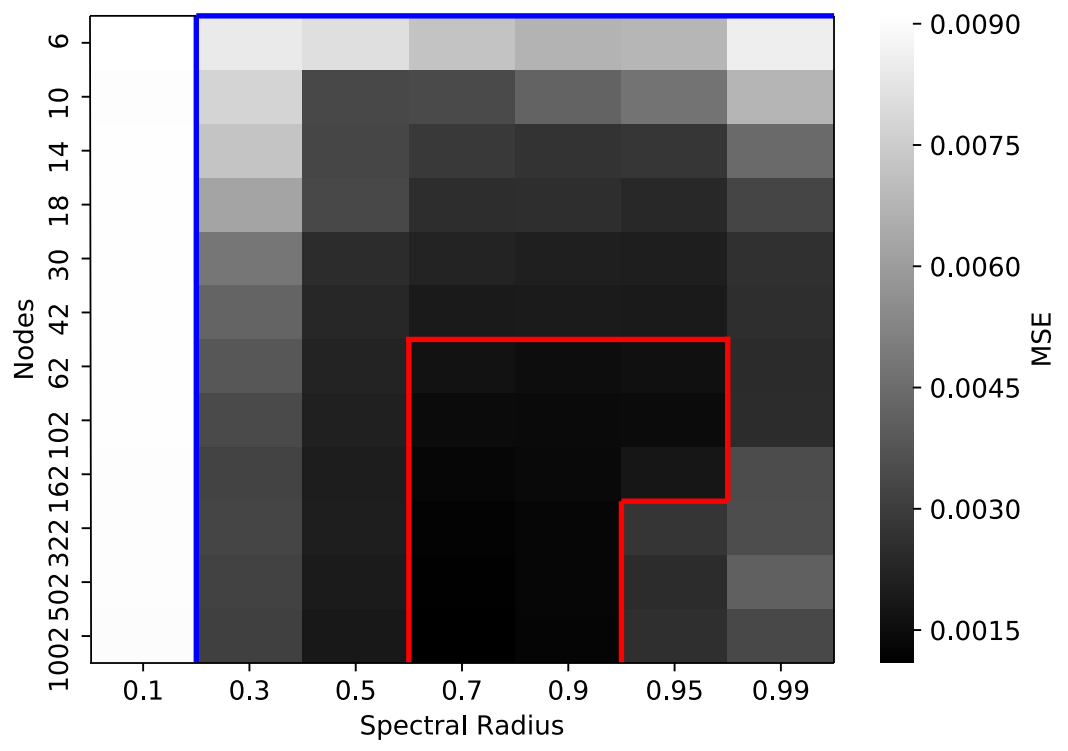

Figure 4.4: MSE of the oscillating neural network on the NARMA-10 task across different $N$ and spectral radius values. The blue line represents the linear prediction model with no memory and the red line shows when the model became better than a linear model with input memory. The model shows a performance improvement as the number of nodes increases and the spectral radius causes significant difference in performance for this task with an

performs better than a linear model with a memory size $=9$ but is outperformed once memory size $=10$.

\section{Discussion}

The experiments showed how having a larger spectral radius improves the performance of the oscillating neural network on the NARMA-10 prediction task. This is different from the results for NARMA-A which performed better with smaller spectral radius but requires a shorter memory. The NARMA-10 has longer term dependencies and so having a larger spectral radius improves its performance on a task, with the optimal value used here being 0.7 and 0.9 . Increasing the spectral radius too high can have a detrimental effect at the larger node values. This indicates that some fading 
memory of previous inputs can improve performance for this task.

The oscillating neural network outperformed the linear memoryless models with 6 nodes and spectral radius $>0.3$, and was able to surpass the linear models with memory once the oscillator had $N=62$ and tuned spectral radius $=0.7$. This could indicate that a larger oscillator can store the necessary memory and that the spectral radius should be tuned to the task.

\section{Table Summary}

A summation of the results from these time series prediction tasks can be seen in Table. 4.1.

\subsection{Sequence Classification}

Next we explore our various sequence classification tasks of recognizing handwritten digits from 0-9, determining whether a splice junction on a gene sequence is an intron/exon or exon/intron junction, and determining the serotype of a dengue virus genome sequence. The goal is to show when and where ESNs provide an improvement over linear classification alone. Logistic regression with regularization is used for the linear models and for the readout layers of the reservoirs.

Additionally the inputs will be passed through a network with the reservoir layer being an oscillating neural network. This classification process is designed with the aim of showing the scale at which these oscillators perform best and strength of connection between the nodes will be scaled using the spectral radius which shows whether fading memory contributes to the performance of the task.

Finally, the reservoir layer is formed using a 3 or 4-way chemical oscillator. The input is fed into the chemical oscillator as scaled concentrations and the chemical 


\begin{tabular}{|c|c|c|c|c|c|c|}
\hline Dataset & $\begin{array}{c}\text { Linear } \\
\text { Regression } \\
\text { memoryless }\end{array}$ & $\begin{array}{c}\text { Linear } \\
\text { Regression } \\
\text { with memory }\end{array}$ & $\begin{array}{c}\text { ESN } \\
\text { Minimum } \\
\text { Complexity }\end{array}$ & $\begin{array}{l}\text { ESN } \\
\text { Best }\end{array}$ & $\begin{array}{c}\text { Oscillating } \\
\text { Neural Network } \\
\text { Best }\end{array}$ & $\begin{array}{l}\text { Chemical } \\
\text { Oscillator }\end{array}$ \\
\hline NARMA-A & $0.42 \pm 0.008$ & 0 & $\mathrm{~N}=3, \mathrm{sp}=0.75$ & $\begin{array}{c}6.1 \times 10^{-6} \\
\mathrm{~N}=100, \mathrm{sp}=0.5\end{array}$ & $\begin{array}{c}3.8 \times 10^{-13} \\
\quad \pm 6 \times 10^{-14} \\
\mathrm{~N}=1002, \mathrm{SR}=0.3\end{array}$ & $0.335 \pm 0.019$ \\
\hline NARMA-10 & $\begin{array}{l}8.8 \times 10^{-3} \\
\pm 6.2 \times 10^{-4}\end{array}$ & $\begin{aligned} & 1.6 \times 10^{-3} \\
\pm & 1.9 \times 10^{-4}\end{aligned}$ & $\begin{array}{l}\mathrm{N}=2, \mathrm{sp}=0.75 \\
\mathrm{~N}=100, \mathrm{sp}=0.1\end{array}$ & $\begin{array}{c}1.8 \times 10^{-4} \\
\mathrm{~N}=2000, \mathrm{sp}=0.1\end{array}$ & $\begin{array}{c}1.1 \times 10^{-3} \\
\pm 3 \times 10^{-5} \\
\mathrm{~N}=1002, \mathrm{SR}=0.7\end{array}$ & $\begin{array}{c}6.6 \times 10^{-3} \\
\pm 2.2 \times 10^{-4}\end{array}$ \\
\hline
\end{tabular}

Table 4.1: Performances of the ESN, oscillating neural network and the chemical oscillator for the NARMA tasks. $\mathrm{sp}=$ sparsity, $\mathrm{SR}=$ spectral radius, the errors are given in MSE. The minimum complexity is when the ESN significantly surpasses the memoryless linear models and linear models with input memory which was represented in the result figures by the blue and red lines. 
output sequences are used by the readout layer for classification. This was done for a point of comparison between the oscillating neural network which is based off of these chemical oscillators.

\subsubsection{MNIST}

This task consists of classifying handwritten digits from 0-9 which are represented as grayscale $28 \times 28$ pixels. The dataset was first flattened and then classified using logistic regression using gradient descent. Using this methodology, an accuracy of 92.5\% can be achieved on MNIST. Some other papers use a linear regression model for classification and have a control value of around 90.5\% [46]. State of the art results on MNIST can be as high as $99.8 \%$ [60]. The 60,000 training examples are used to train the network and the 10,000 test examples are used for reporting accuracies.

\subsubsection{Echo State Networks}

The aim of this exploration is to demonstrate an improvement over linear models using a minimum complexity of reservoir in an ESN. For the purposes of this experiment the MNIST dataset is fed column by column with each of the rows as input channels. The results of the accuracy is shown in Fig. 4.5. A spectral radius of 0.9 was fixed for the experiments and the sparsity and size of the networks was varied.

Some experiments were also done using only the final output of the reservoir for classification these results can be seen in Fig. 4.6.

\section{Discussion}

Using the ESNs, an improvement over linear classification accuracy could be achieved using the settings $N=20$ and sparsity $=0.1$. This is results in a smaller readout layer of $20 \times 28$ rather than $28 \times 28$ in the baseline model. It should be 


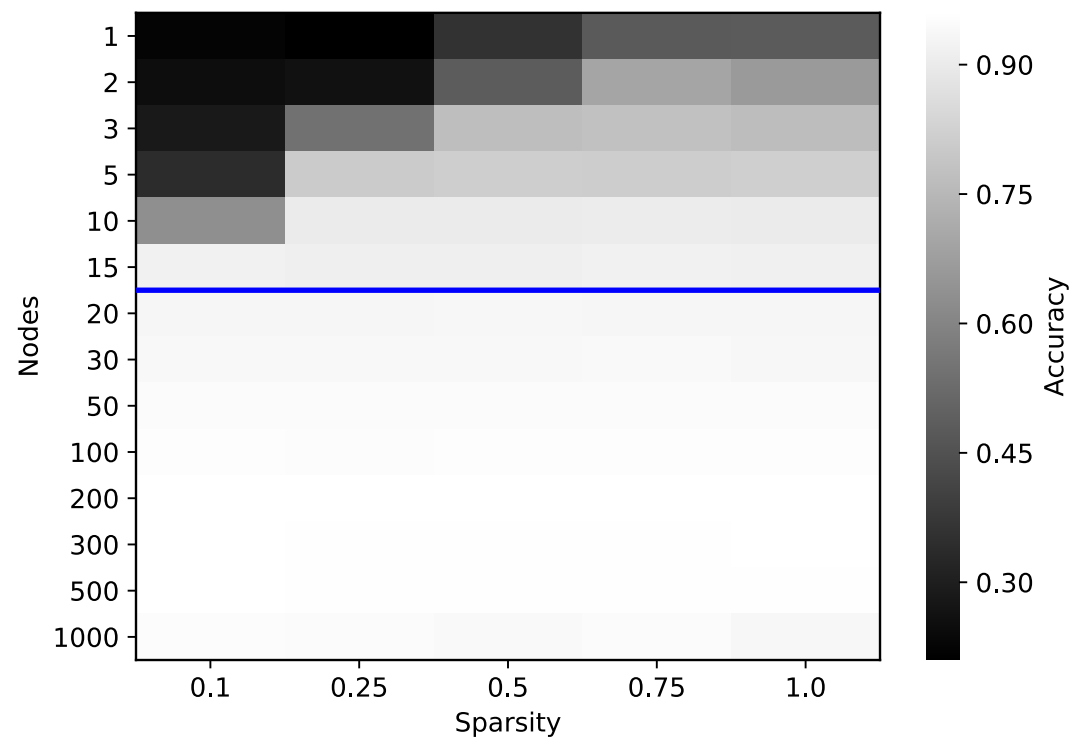

Figure 4.5: Classification accuracy for MNIST using ESNs. The ESN shows an improvement over linear classification, the blue line, with 20 nodes and a sparsity of 0.1. There is not a significant trend in different performances for sparsity at the higher values of nodes, however at smaller node values, too much sparsity can lead to a strong dip in performance as incomplete networks can form.

noted that MNIST images are not orthogonal and have black space around them which can be cropped to reduce their dimensions. However, based on this research it can be noted that even a limited amount of random non-linear projections improves the ability of the input to be linearly classified. It was found that the classification capability of the reservoir does increase with its size, however improvements were not significant above 500 nodes although further explorations into regularization methods and more hyperparameter tuning could improve the model.

An issue which affects accuracy for the small networks with low sparsity values is that the small level of connectivity likely causs incomplete networks with parts which are unconnected from the rest of them hurting performance and if no connections are formed an accuracy of around 0.1 occurs which for MNIST is equivalent to random 


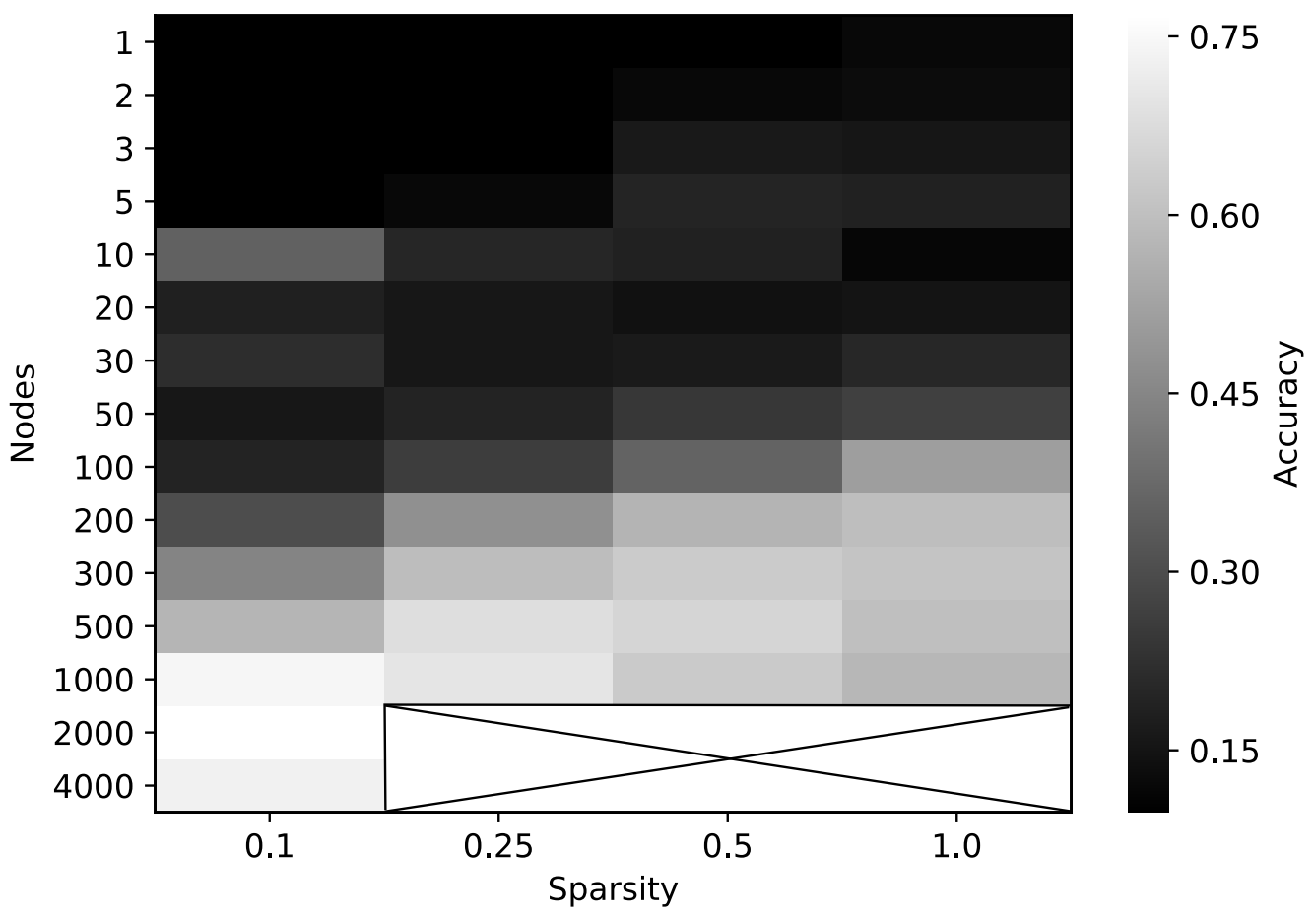

Figure 4.6: Classification accuracy on MNIST for ESN using only the final readout value of the reservoir nodes. A significant reduction in performance was found when this constraint was placed on the model falling from $96.5 \%$ accuracy with 1000 nodes in Fig. 4.5 to $74.3 \%$ in this model. Although this does reduce the output from being $N \times 28$ to being $N \times 1$ which reduces the readout complexity. The ' $\mathrm{X}$ ' marks no accuracy value at the higher node values.

guessing.

While a smaller ESN reservoir with less nodes can sometimes perform well, there are larger standard deviations for the results which indicate that it is susceptible to the random initialization of the input reservoir matrix. Having a larger reservoir can compensate for this sensitivity.

For the larger networks regularization began to play a significant role as the difference between training and test accuracy increased. This was counteracted using dropout $=0.05$ as well exploration of using $L 1$ and $L 2$ regularization of the weights. 
For a point of reference, we trained a simple recurrent neural network and LSTM network with 100 nodes which achieved an accuracy of $98.5 \%$ and $99.1 \%$ respectively.

When using only the final output from reservoir nodes, a significant decrease in performance was observed for this task. For $N=1000$ and sparsity $=0.1$, the performance of the ESN dropped from $96.1 \%$ to $74.3 \%$. This is likely due to loss of information from the decaying memory of the network, which causes earlier inputs to gradually decay over time. Additionally the final few columns of MNIST digits tend to be 0 values which would dampen the node outputs. In classification tasks, it can be the case that the more recent inputs are not proportionately more useful for the desired task. A key feature may occur early on in the sequence and the model needs to be able to identify this feature in order to more accurately classify the sequence.

\subsubsection{Oscillator Networks}

We explored the MNIST task using the oscillating neural network across different node sizes and spectral radius. The accuracy results can be seen in Fig. 4.7.

Using the 3-chemical oscillator as a projection kernel an accuracy of $90.5 \%$ was achieved which under performs compared to linear models alone, however this is without full optimization of the chemical reaction rates, input scaling, and oscillator size.

\section{Discussion}

From the results in Fig. 4.7, it can be seen that an oscillator can function as an effective reservoir to improve the classification rate beyond that which can be achieved through just the readout layer. With the performance of the oscillating neural network crossing the linear baseline at around 30 nodes.

This experiment was also done using the input format of inputting the MNIST 


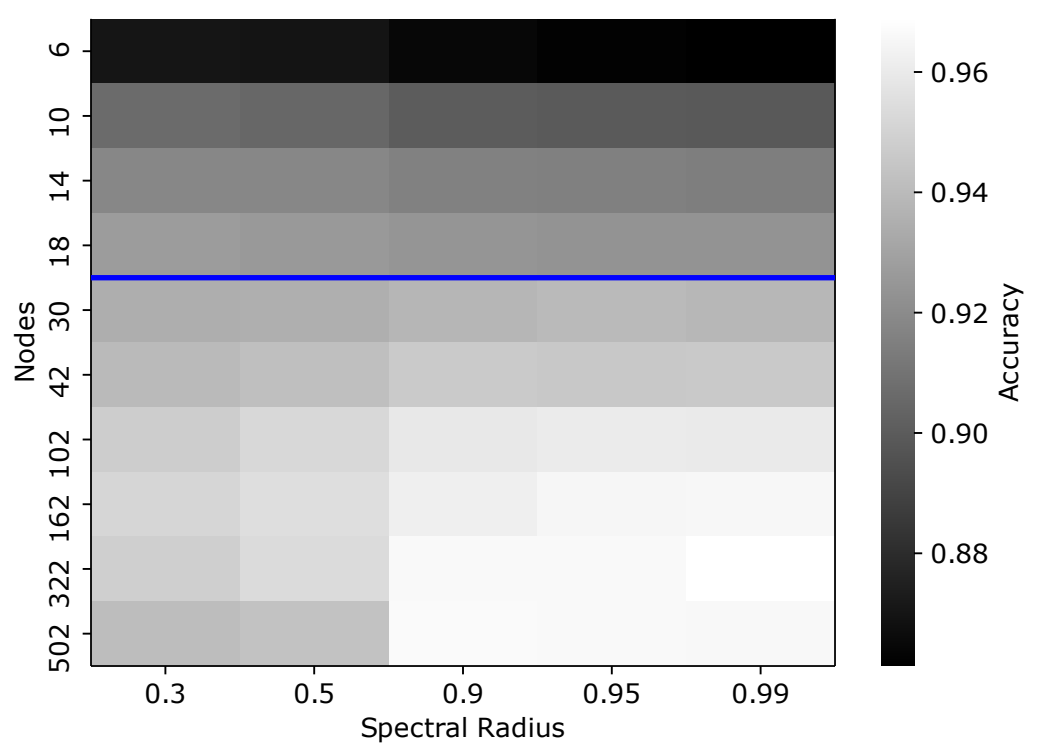

Figure 4.7: Classification accuracy across the different parameters using the neural oscillating network. The accuracy improves as the size of the network grows crossing the blue line, which indicates the threshold for a linear classifier, when $N=30$. There is a significant trend at the larger graph size that having a larger spectral radius improves the performance

in a single pixel at a time was explored as this was the technique involved in other work. Performance for a neural oscillating network with 6 nodes could achieve $95.6 \%$ accuracy, this may be due to the increase in dimensionality from the different method of input.

For the accuracy with the chemical oscillator, the values $A=B=C=1$ were used for initial conditions, and the reaction rates of $k 1=k 2=k 3=1$. The time series was fed in as a scaled concentraion value of $A$ with an input value with scaling factor 0.1. This could still be optimized in terms of reaction weights or input scaling. These initial results show that the oscillator is capable of still achieving over a $90 \%$ accuracy for this task. Previous experiments for chemical implementation on this task [3] removed $10-16 \%$ of the sample set in order to have a separable output layer as they required a 
probability separation of 0.3 for their class output between two classes. Base on the experiments with the oscillating neural network, a larger chemical oscillator as well as tuning the reaction rates may be a way of improving the performance.

\subsubsection{Splice Junction Gene Sequences}

In this section the Splice Junction Gene Sequences are classified into the three classes based on whether they are Intron/Exon, Exon/Intron junctions, or Neither. In order for these 60bp gene sequence dataset to be input into the model, it first was one-hot encoded for each nucleotide base pair. The baseline performance of logistic regression on this task was found to be $94 \%$. Previous best performance in the literature using CNNs was $96.2 \%$ [62]. These were reported using 10-fold cross-validation across three different seeded initializations of the reservoirs.

\subsubsection{Echo State Networks}

We used an input scaling $U[-1,1]$ for the echo state networks and exploration of the different number of nodes and sparsity within the reservoir was performed. The results for these experiments can be seen in Fig. 4.8 .

\section{Discussion}

Using the full reservoir readout, an improvement over linear models was achieved with 5 nodes and a sparsity $>0.25$. This projects the input into a higher temporaldimensional which can then be more easily classified. There was diminishing returns on performance as the reservoir became larger which could indicate that a better architecture or regularization technique was needed. Additionally some of the accuracy means have higher variability than was desired which could be due to the small dataset size of $3 \mathrm{~K}$. Other regularization or training techniques which are tailored to 


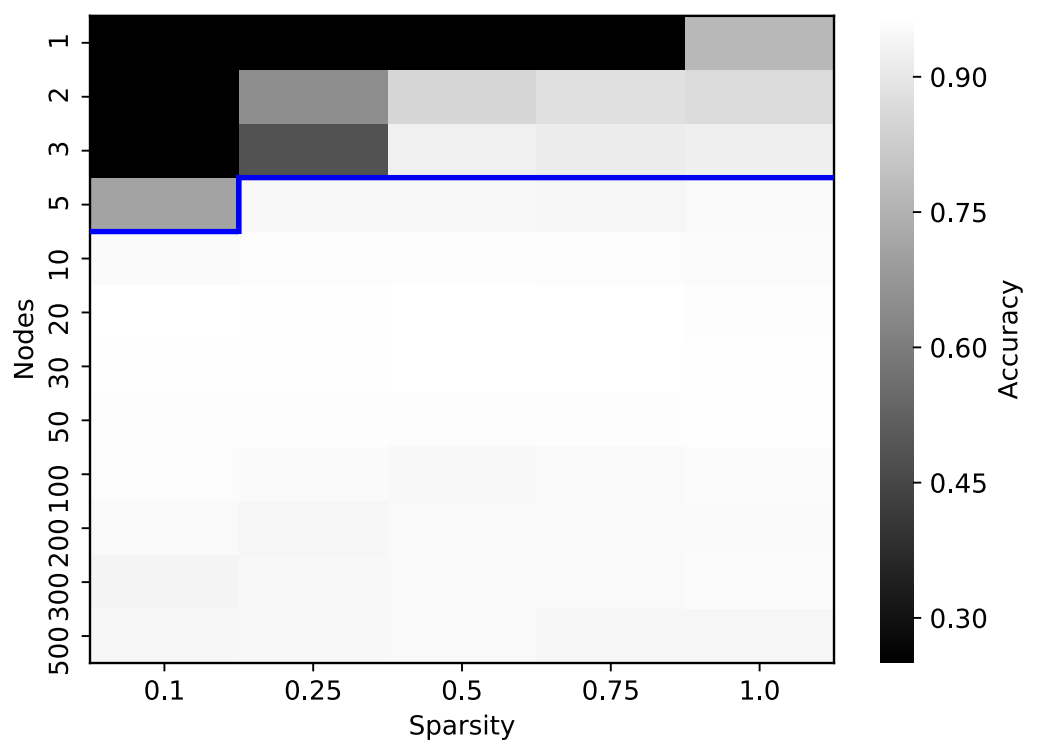

Figure 4.8: Classification accuracy of the ESNs on the splice junction gene sequences across the different sparsity and $N$ values. Increasing network size to above $N=$ 5 can improve performance above the linear baseline, represented by the blueline. Diminishing returns was found as the network grew larger.

this size of dataset could improve variance and generalization of the models.

The best performance was found using and ESN of $N=20$ and sparsity $=0.5$ which achieved an accuracy of $96.8 \pm 0.005$. For reference an LSTM with $N=100$ was trained on this dataset and achieved $97.2 \%$ which is slightly better then the best ESN but only by a small amount $0.4 \%$. Based on more data exploration, we see that the sequences themselves do contain levels of noise such as missing and unknown nucleotides in many of the sequences which make classification difficult. Gathering more clean data would likely improve this performance which can be said of most accuracy performances.

In this case it is also important to explore the confusion matrix for the dataset which is shown in Fig. 4.9 as there is some imbalance in the dataset, $I E$ : 25\%, EI : 25\%, Neither: 50\%. Identifying where the model struggles can be informative for 
improving the model.

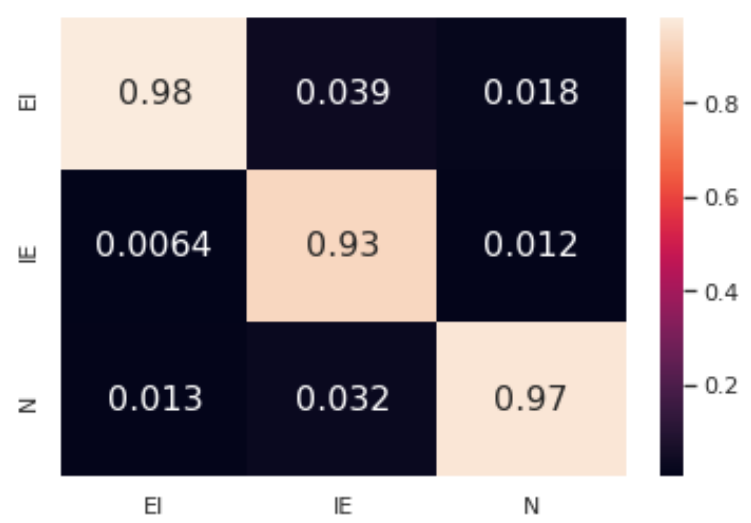

Figure 4.9: Confusion matrix for the splice junction gene sequences of the ESN with $N=10$, sparsity $=1.0$. The predicted class is on the y-axis and actual is listed on the x-axis, the columns are normalized and sum to 1.0. The intron/exon junctions appear to be the most difficult to classify and get about equally confused with the other classes.

\subsubsection{Oscillator Networks}

The gene sequences were passed into the neural oscillator network with an input weight matrix $U[-1,1]$. The network was explored using different size and spectral radius values the accuracy results are plotted in Fig. 4.10.

The splice genomes were fed into the modified 4-way oscillating chemical reaction network described in the Section 2.3.2 which has a four chemical species such that for each nucleotide a scaled concentration of 0.1 was fed into the network for each chemical in the oscillator e.g. an A nucleotide is encoded as 0.1 of chemical A and a T nucleotide is encoded as 0.1 of chemical B.

The final result for the chemical oscillator was $94.3 \% \pm 0.008$ which is comparable to the logistic regression model. This does however show that there is not a significant classification loss, when projecting the sequences through this simulated chemical oscillator. 


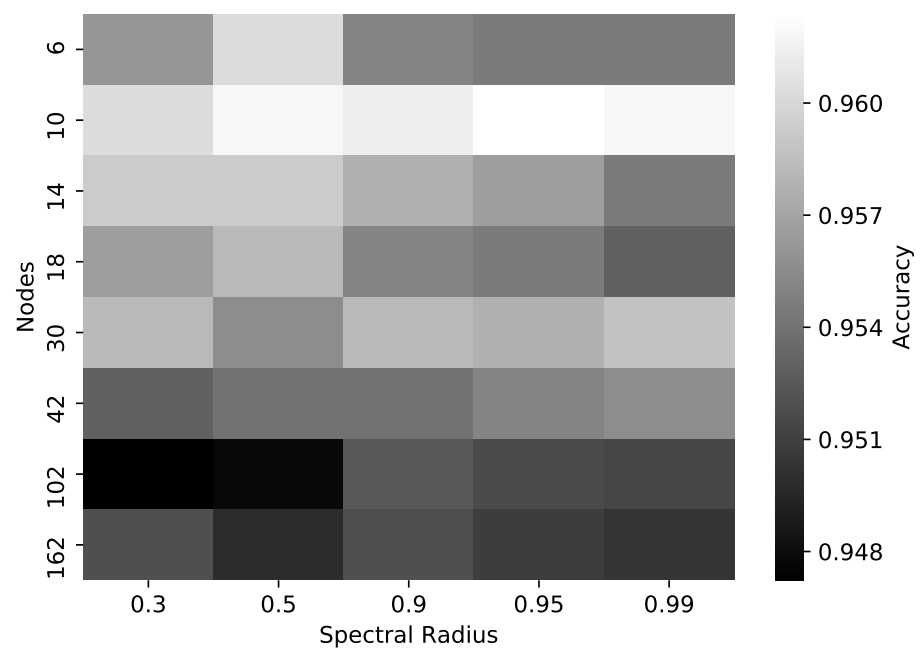

Figure 4.10: Classification accuracy for the oscillating neural network on the splice junction gene sequences across the different parameters of spectral radius and nodes. All of the settings performed as well as the linear model once the variation is accounted for.

\section{Discussion}

This task of splice junction gene classification was used as an initial exploration of applying these techniques to a genomic task. This task can already be accomplished with a high accuracy of around $94 \%$ using logistic regression methods. This is likely due to the sequences being aligned with the junctions being at the middle index of the sequence which is one of the key issues in genomic analysis and why alignment-free solutions are used such as k-mer extraction.

For the oscillating neural network, there was a similar performances across the different settings only ranging by about $2 \%$ from the worst to the best classification accuracy. The smaller networks can have higher variability in their performance which, but can achieve a slightly higher accuracy. It could be that the input scaling needs to be tuned for each particular size of network which could be negatively affecting accuracy for larger networks which have more overall input. 


\subsubsection{Dengue Virus Genome}

For the dengue virus (DV) serotype classification task, a subsampled genome sequence of length 200 base pairs, was taken from the beginning of the genome which contains the UTR and the capsid genome sequences in the first 500 nucleotides in the genome. These subsequences will be classified into the 4 different DV serotypes (DENV-1, DENV-2, DENV-3, DENV-4)

This task involves a more difficult genome classification challenge due to the lack of alignment and the variability in the beginning section of the genome sequence. This requires the network to extract the sequence dependent information which is present in genomes.

One thing to note in this dataset is the categorical imbalance which is why the

accuracy for the poor performing reservoirs bottoms out at around $40 \%$ which is the concentration of DENV-1 in the dataset.

Additionally, due to the subsampling, the dataset grows considerably larger from 3,625 to 53,475. From each genome section 15 subsamples were taken which gives an average 6 fold coverage for each base pair.

The baseline logistic regression model with regularization for this task could achieve $82.8 \%$ classification accuracy.

\subsubsection{Echo State Networks}

Here are presented the results of using ESNs with varying sizes and sparsity values on this task. The spectral radius was set to 0.9 and the input values were $U[-1,1]$. 


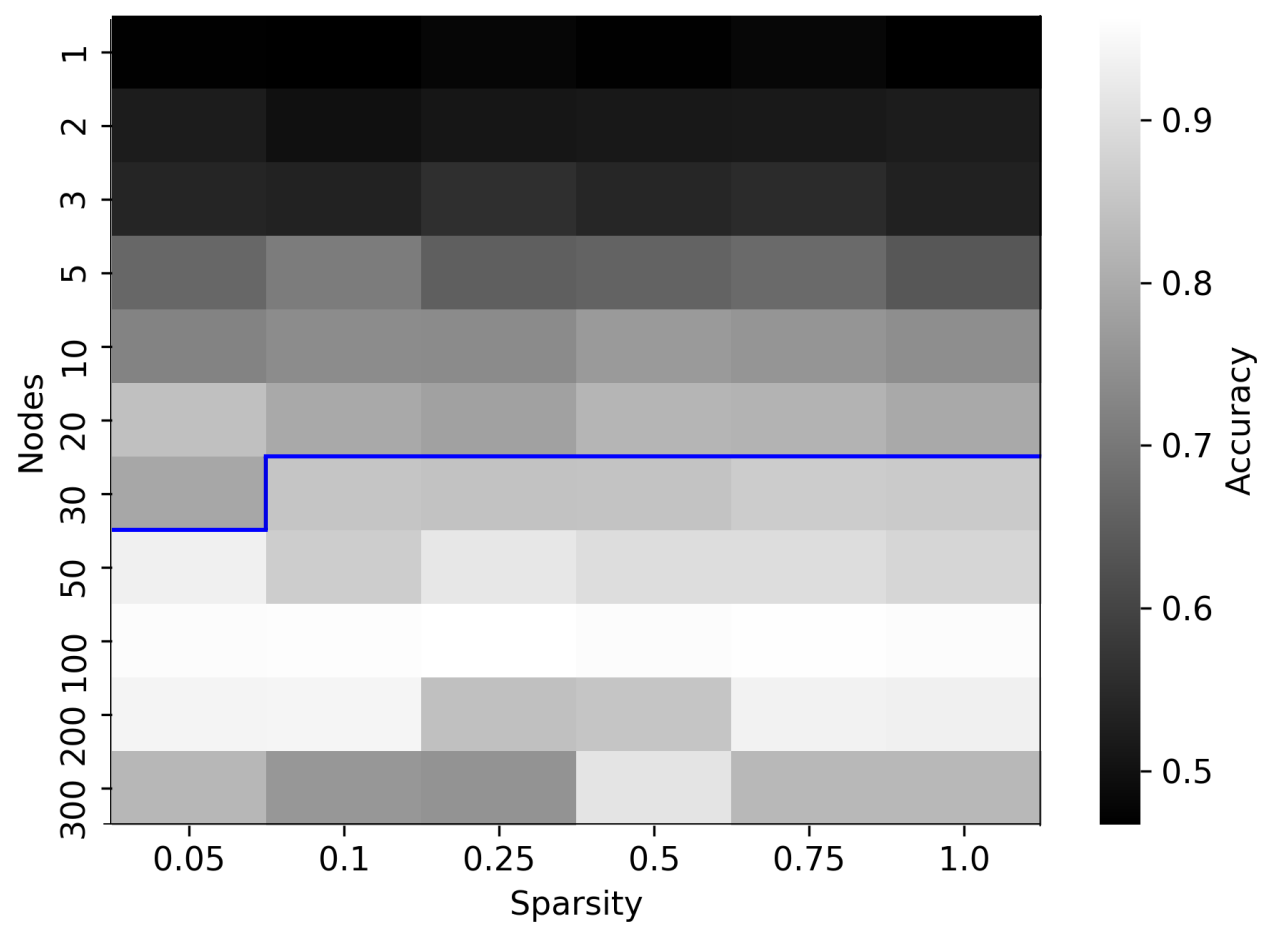

Figure 4.11: Classification accuracy for the ESN on the dengue virus genome subsequences. The blue line indicates linear model classification threshold. The ESN able to extract meaningful features from the 200bp sequences with $N=30$ and sparsity $=0.1$, the performance was best at $N=100$ while at higher $N$ values the network seemed to suffer from being caught in a local minimum when training the output and had higher variation which may be solved by adjusting hyperparameters such as adjusting batch size.

\section{Discussion}

The ESN was able to improve the classification rates of the subsampled strands with $N=30$ and achieved a top accuracy of $96.4 \%$ at $N=100$ and sparsity $=0.5$ resulting in a $13.6 \%$ improvement over linear baseline in terms of means. When $N$ became larger than $N=100$ some loss in performance was experienced, this could be due to the need to optimize the inputs for the size of the reservoir or the readout layer being stuck in a local minima. 
One thing to note is that there can be more differentiation between one serotype to another. This can be shown in a phylogenetic trees of DV which shows this splitting. By examining here a confusion matrix of the classification in Fig. 4.12 in some cases the classifier performs well on certain classes, but then struggles with differentiating DENV-2 and DENV-3.

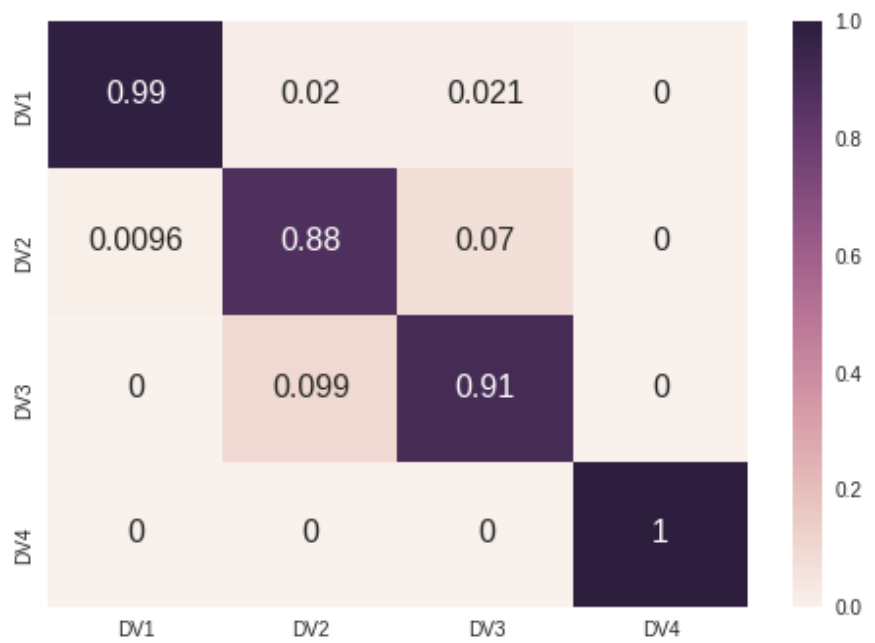

Figure 4.12: Confusion matrix showing the performance of a $\mathrm{ESN}$ with $N=100$, sparsity $=1.0$ on classifying the beginning $200 \mathrm{bp}$ of the dengue genome sequence. This shows that there may be similarities between DENV-2 and DENV-3 which are more difficult for this classifier to identify.

This could aid in evaluating what statistical property the reservoir is learning in order to differentiate between the different dengue serotypes. It may also have to do with the phylogenetic similarity between different serotypes.

Additional work and experimentation for this data set could involve the use of sampling of the entire genome which would generate a dataset size of around 3 million samples. Exploration of this task using a trained LSTM network with 100 neurons achieved an accuracy rate of $99.7 \%$. This shows that there is some features which can be extracted and used to classify, but the large amount of variability in the ESN and the differing structure may require more search. This issue of identifying out of 
alignment sequences in a genome is typically addressed in classifiers using the k-mer extraction on the strand and then classifying it accordingly [55].

\subsubsection{Oscillator Networks}

The DV genomes were fed through the oscillating neural network and node outputs were used for the readout layer. The input matrix was again initialized using $U[-1,1]$ distribution. The results of the network are shown in Fig. 4.13.

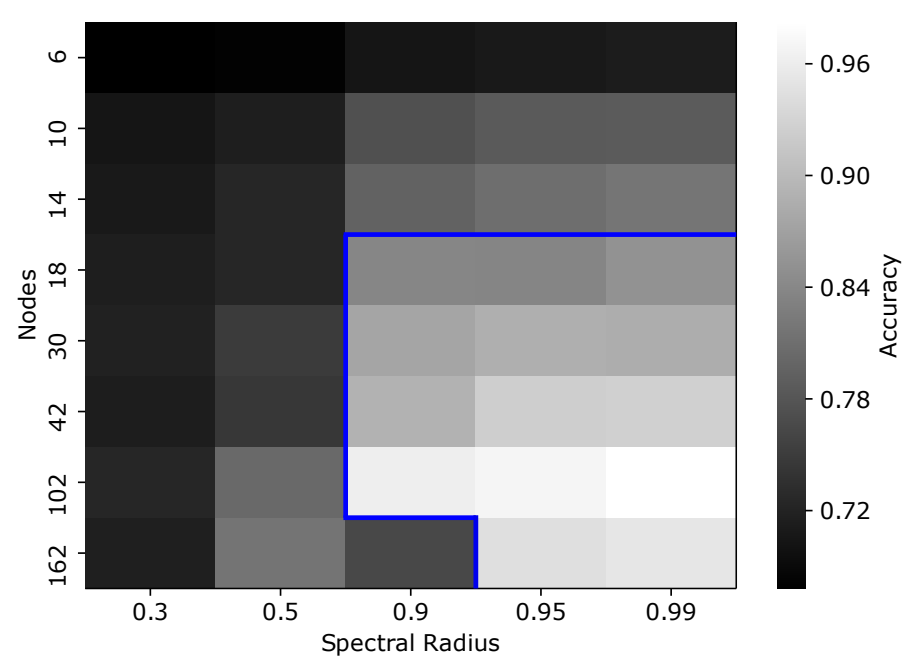

Figure 4.13: Classification accuracy for DV serotype across the different oscillating neural network sizes and spectral radii. The blue line indicates the threshold for linear classification. The network performs best with the larger nodes and the highest spectral radius.

The 4-way chemical oscillator was used for this task and with some input scaling adjustments the accuracy of $70.3 \%$ was achieved. This is significantly lower than the linear baseline which could indicate that the oscillator is causing noise for the readout. 


\section{Discussion}

The oscillating neural network was able to improve above the linear baseline with a network of size $N=18$ and a spectral radius of 0.9 . The performance of this oscillating neural network was slightly better than the ESN with $N=100$ by having a spectral radius $=0.99$ instead of 0.9 . This shows that in order to classify the genome

sequences well, a longer memory of past inputs is helpful. Identifying this type of long term dependency in a viral genome sequence could aid in analysis of methods for identifying these viruses and might provide insight into some form of treatment if some gene pathway could be disrupted in certain regions of the genome.

The chemical oscillator struggled with this task performing even worse than the logistic regression model. The addition of lack of alignment could cause this dip in performance which may indicate that the size of the reservoir needs to be increased in order to achieve better results.

\section{Table Summary}

A summation of the results for the classification tasks can be seen in Table. 4.2. 


\begin{tabular}{|c|c|c|c|c|c|c|}
\hline Dataset & Classes & Linear & $\begin{array}{c}\text { ESN } \\
\text { Minimum } \\
\text { Complexity }\end{array}$ & $\begin{array}{l}\text { ESN } \\
\text { Best }\end{array}$ & $\begin{array}{c}\text { Oscillating } \\
\text { Neural Network } \\
\text { Best }\end{array}$ & $\begin{array}{l}\text { Chemical } \\
\text { Oscillator }\end{array}$ \\
\hline MNIST & 10 & $0.925 \pm 0.001$ & $\mathrm{~N}=10, \mathrm{sp}=0.1$ & $\begin{array}{c}0.961 \pm 0.001 \\
\mathrm{~N}=1000, \mathrm{sp}=0.1\end{array}$ & $\begin{array}{c}0.969 \pm 0.003 \\
\mathrm{~N}=322, \mathrm{SR}=0.99\end{array}$ & $0.906 \pm 0.001$ \\
\hline Splice Junction & 3 & $0.94 \pm 0.003$ & $\mathrm{~N}=5, \mathrm{sp}=0.25$ & $\begin{array}{l}0.968 \pm 0.002 \\
\mathrm{~N}=20, \mathrm{sp}=0.5\end{array}$ & $\begin{array}{l}0.962 \pm 0.003 \\
\mathrm{~N}=10, \mathrm{SR}=0.5\end{array}$ & $0.94 .2 \pm 0.002$ \\
\hline DV & 4 & $0.828 \pm 0.002$ & $\mathrm{~N}=30, \mathrm{sp}=0.1$ & $\begin{array}{c}0.964 \pm 0.007 \\
\mathrm{~N}=100, \mathrm{sp}=0.5\end{array}$ & $\begin{array}{c}0.982 \pm 0.002 \\
\mathrm{~N}=102, \mathrm{SR}=0.99\end{array}$ & $0.706 \pm 0.005$ \\
\hline
\end{tabular}

Table 4.2: Classification accuracy of the ESN, oscillating neural network and the chemical oscillator for the classification tasks. $\mathrm{sp}=$ sparsity, $\mathrm{SR}=$ spectral radius. The minimum complexity is when the ESN significantly surpasses the logistic regression model represented in the results figures by the blue lines. 


\section{Conclusion}

The goal of this thesis was to attempt to leverage the tools developed in the field of reservoir computing to improve the scaling capacity of DNA based circuits for accomplishing complex tasks.

This was done by exploring a key research segment of reservoir computing that uses recurrent neural networks known as echo state networks. These ESNs were demonstrated for the sequence prediction tasks of NARMA-A and NARMA-10 and the sequence classification tasks of MNIST, splice junction gene sequences, and dengue virus genome serotype. Since implementing more complex systems in a DNA circuit will be more difficult, a key aim was to identify the place at which the addition of this reservoir layer provides an improvement over linear baseline results with minimum size and sparsity. These results can also aid in identifying network complexity trade-offs for solving a particular task where some point of diminishing returns exists.

Using ESNs for the NARMA-A, an improvement over the memoryless linear model was achieved with $N=3$ and sparsity $=0.75$ and for NARMA-10 an improvement over the memoryless linear model was achieved at $N=2$ with a sparsity $=0.75$ and an improvement over a linear model with memory of inputs was achieved at $N=100$ and sparsity $=0.1$.

For the classification tasks, we found using an ESN provided improvements over linear baselines for MNIST at $N=10$, sparsity $=0.1$; for splice junction gene sequence at $N=5$, sparsity $=0.25$; and for the DV genome serotyping at $N=30$, sparsity $=0.1$. For the ESN's performance, the size of the reservoir, $N$, had a much more significant impact on performance than sparsity. The networks performed similarly 
whether they had a sparsity of 0.1 or fully connected with a sparsity $=1.0$ except for small values of $N$ where sparsity played a stronger role.

These results with the ESNs showed that different levels of complexity could be used and tuned to each task and, in many cases, can be improved by using a very small, sparsely connected ESN. This indicates that other reservoir layers with comparable dynamics and complexity could perform well on the tasks.

A difficulty for these results is that the readout layer was trained with access to all of the reservoir's output values across time, which requires a large number of readout layer weight connections. For the MNIST task, a sharp drop of around 20\% accuracy occurred when only the final output of the ESN reservoir was used. Some future work into reservoir output subsampling or adding a dimensionality reduction step before the readout layer could reduce the complexity of these networks by reducing the number of connections to the readout layer.

To bridge the gap between ESNs and oscillating chemical reaction networks, an oscillating recurrent neural network was used as a reservoir layer. This oscillating neural network's performance was evaluated on previous tasks with different size and spectral radius $(S R)$ values. The experimental results showed that larger sized networks performed better, but that the optimum spectral radius varied between tasks. For NARMA-A, an $S R=0.3$ gave the best performance, whereas on NARMA10 an $S R=0.7$ gave the best performance. For MNIST and on the dengue genome classification tasks, an $S R=0.99$ gave the highest accuracy. This is intended to demonstrate that the connection rates between nodes affects the performance of the network on the task. For CRNs, this may give insight into the level of chemical dynamics necessary for an effective reservoir layer in terms of the reaction rates or the rate at which to influx and remove chemical concentrations for a particular task.

We demonstrated using the oscillating neural network that an oscillator can work 
as an effective projection kernel for the various tasks. An issue of translating this to chemical oscillators is that the tanh function is used, and therefore the node's output values can be from -1 to 1 . The concept of negative chemical concentrations may not be precisely transferable. One perspective of adjusting translation could be that the node activation represents the changes of the concentrations over time or their concentration relative to an equilibrium value.

Finally, a simulated chemical oscillator was used as the reservoir layer. A 3 and 4-way chemical oscillator was used with the 4-way being used on the gene sequence tasks due to the input encoding. By using a chemical oscillator as a reservoir, an improvement in MSE was achieved for the sequence tasks. Improving NARMA-A from $\mathrm{MSE}=0.42$ to 0.33 and improving NARMA-10 from a baseline $\mathrm{MSE}=8.8 \times 10^{-3}$ to $6.6 \times 10^{-3}$. Using this chemical oscillator as a reservoir, we were able to achieve a classification accuracy of $90.6 \%, 94.2 \%$, and $70.6 \%$ on the MNIST, splice junction gene sequences, and dengue genome serotypes classification tasks respectively.

These accomplishments with the chemical oscillator on the tasks of genome classification are not an improvement over linear baseline models, but are better than random guessing and could be useful due to their potential for being implemented with DNA logic in situ.

Our design utilizes labeled genome data, which is input as a multivariate timeseries. Translating this input into the wet-lab implementation would require that the genome sequence be input one base at a time, each base one-hot encoded as a particular oligonucleotide. We proposed here some methods which could be used for this input implementation to the reservoir.

Implementing the CRN reservoir using a pool of oligonucleotide species of sufficient complexity should be possible using a system of DNA toe-hold strand displacement cycles. An open microfluidic reactor could also be used to control chemical species 
flow into and out of the reservoir.

While the exact implementation may be technically challenging, based on these initial results, it seems possible that some form of reservoir computing system could be used as an effective pathogen classifier. Further research may lend insights into ways of improving these DNA reservoir networks. 


\section{References}

[1] A. Hjelmfelt, E. D. Weinberger, and J. Ross, "Chemical implementation of neural networks and turing machines," Proceedings of the National Academy of Sciences, vol. 88, no. 24, pp. 10983-10987, 1991.

[2] R. Lopez, R. Wang, and G. Seelig, "A molecular multi-gene classifier for disease diagnostics," Nature Chemistry, vol. 10, no. 7, p. 746, 2018.

[3] K. M. Cherry and L. Qian, "Scaling up molecular pattern recognition with dnabased winner-take-all neural networks," Nature, vol. 559, no. 7714, p. 370, 2018.

[4] L. Qian, E. Winfree, and J. Bruck, "Neural network computation with dna strand displacement cascades," Nature, vol. 475, no. 7356, p. 368, 2011.

[5] G. Dion, S. Mejaouri, and J. Sylvestre, "Reservoir computing with a single delaycoupled non-linear mechanical oscillator," Journal of Applied Physics, vol. 124, no. 15, p. 152132, 2018.

[6] H. Jaeger, Reservoir computing approaches to recurrent neural network training.

[7] A. Goudarzi, M. R. Lakin, and D. Stefanovic, "DNA reservoir computing: a novel molecular computing approach," in International Workshop on DNA-Based Computers, pp. 76-89, Springer, 2013.

[8] D. Soloveichik, G. Seelig, and E. Winfree, "DNA as a universal substrate for chemical kinetics," Proceedings of the National Academy of Sciences, vol. 107, no. 12 , pp. 5393-5398, 2010. 
[9] N. Srinivas, J. Parkin, G. Seelig, E. Winfree, and D. Soloveichik, "Enzyme-free nucleic acid dynamical systems," Science, vol. 358, no. 6369, p. eaal2052, 2017.

[10] W. Yahiro, N. Aubert-Kato, and M. Hagiya, "A reservoir computing approach for molecular computing," in Artificial Life Conference Proceedings, pp. 31-38, MIT Press, 2018.

[11] U. S. Bhalla, "Understanding complex signaling networks through models and metaphors," Progress in Biophysics and Molecular Biology, vol. 81, pp. 45-65, Jan. 2003.

[12] L. M. Adleman, "Molecular computation of solutions to combinatorial problems," Science, pp. 1021-1024, 1994.

[13] R. S. Braich, N. Chelyapov, C. Johnson, P. W. Rothemund, and L. Adleman, "Solution of a 20-variable 3-SAT problem on a DNA computer," Science, vol. 296, no. 5567, pp. 499-502, 2002.

[14] Y. Benenson, "Biomolecular computing systems: principles, progress and potential," Nature Reviews Genetics, vol. 13, no. 7, p. 455, 2012.

[15] F. Wang, H. Lv, Q. Li, J. Li, X. Zhang, J. Shi, L. Wang, and C. Fan, "Implementing digital computing with dna-based switching circuits," Nature Communications, vol. 11, no. 1, pp. 1-8, 2020.

[16] M. Lukoševičius and H. Jaeger, "Reservoir computing approaches to recurrent neural network training," Computer Science Review, vol. 3, no. 3, pp. 127-149, 2009. 
[17] B. Jones, D. Stekel, J. Rowe, and C. Fernando, "Is there a liquid state machine in the bacterium escherichia coli?," in 2007 IEEE Symposium on Artificial Life, pp. 187-191, IEEE, 2007.

[18] N. Caporale and Y. Dan, "Spike TimingDependent Plasticity: A Hebbian Learning Rule," Annual Review of Neuroscience, vol. 31, pp. 25-46, July 2008.

[19] W. S. McCulloch and W. Pitts, "A logical calculus of the ideas immanent in nervous activity," The bulletin of mathematical biophysics, vol. 5, no. 4, pp. 115$133,1943$.

[20] R. Hecht-Nielsen, "Theory of the backpropagation neural network," in Neural networks for perception, pp. 65-93, Elsevier, 1992.

[21] Y. S. Abu-Mostafa, M. Magdon-Ismail, and H.-T. Lin, Learning from data, vol. 4. AMLBook New York, NY, USA:, 2012.

[22] A. Krizhevsky, I. Sutskever, and G. E. Hinton, "Imagenet classification with deep convolutional neural networks," in Advances in neural information processing systems, pp. 1097-1105, 2012.

[23] K. He, X. Zhang, S. Ren, and J. Sun, "Deep residual learning for image recognition," in The IEEE Conference on Computer Vision and Pattern Recognition (CVPR), June 2016.

[24] CS231n Convolutional Neural Networks for Visual Recognition, http://cs231n. github.io/convolutional-networks/, Accessed: 12-13-2019.

[25] S. J. Pan and Q. Yang, "A survey on transfer learning," IEEE Transactions on knowledge and data engineering, vol. 22, no. 10, pp. 1345-1359, 2009. 
[26] D. Quang and X. Xie, "Danq: A hybrid convolutional and recurrent deep neural network for quantifying the function of DNA sequences," Nucleic Acids Research, vol. 44, p. gkw226, 042016.

[27] Y. Bengio, P. Simard, P. Frasconi, et al., "Learning long-term dependencies with gradient descent is difficult," IEEE transactions on neural networks, vol. 5, no. 2, pp. 157-166, 1994.

[28] S. Hochreiter, M. Heusel, and K. Obermayer, "Fast model-based protein homology detection without alignment," Bioinformatics, vol. 23, no. 14, pp. 1728-1736, 2007.

[29] F. A. Gers, J. Schmidhuber, and F. Cummins, "Learning to forget: Continual prediction with lstm," 1999.

[30] Understanding LSTM Networks - colah's blog, https://colah.github.io/ posts/2015-08-Understanding-LSTMs/, Accessed:12-10-2019.

[31] R. Devaney, An introduction to chaotic dynamical systems. CRC Press, 2018.

[32] N. Bertschinger and T. Natschlger, "Real-time computation at the edge of chaos in recurrent neural networks," vol. 16, no. 7, pp. 1413-1436.

[33] H. Jaeger, "The echo state approach to analysing and training recurrent neural networks-with an erratum note," Bonn, Germany: German National Research Center for Information Technology GMD Technical Report, vol. 148, no. 34, p. 13, 2001.

[34] J. J. Steil, "Backpropagation-decorrelation: online recurrent learning with o(n) complexity," in 2004 IEEE International Joint Conference on Neural Networks (IEEE Cat. No.04CH37541), vol. 2, pp. 843-848 vol.2. 
[35] A. Jalalvand, G. V. Wallendael, and R. V. D. Walle, "Real-time reservoir computing network-based systems for detection tasks on visual contents," in 2015 7th International Conference on Computational Intelligence, Communication Systems and Networks, pp. 146-151, IEEE.

[36] I. B. Yildiz, H. Jaeger, and S. J. Kiebel, "Re-visiting the echo state property," Neural Networks, vol. 35, pp. 1-9, 2012.

[37] S. Bai, J. Z. Kolter, and V. Koltun, "An empirical evaluation of generic convolutional and recurrent networks for sequence modeling," arXiv preprint arXiv:1803.01271, 2018.

[38] G. Tanaka, T. Yamane, J. B. Héroux, R. Nakane, N. Kanazawa, S. Takeda, H. Numata, D. Nakano, and A. Hirose, "Recent advances in physical reservoir computing: a review," Neural Networks, vol. 115, pp. 100-123, 2019.

[39] W. Maass, T. Natschläger, and H. Markram, "Real-time computing without stable states: A new framework for neural computation based on perturbations," Neural Computation, vol. 14, no. 11, pp. 2531-2560, 2002.

[40] M. Davies, N. Srinivasa, T.-H. Lin, G. Chinya, Y. Cao, S. H. Choday, G. Dimou, P. Joshi, N. Imam, S. Jain, et al., "Loihi: A neuromorphic manycore processor with on-chip learning," IEEE Micro, vol. 38, no. 1, pp. 82-99, 2018.

[41] A. Blum, "Random projection, margins, kernels, and feature-selection," in Subspace, Latent Structure and Feature Selection (C. Saunders, M. Grobelnik, S. Gunn, and J. Shawe-Taylor, eds.), vol. 3940, pp. 52-68, Springer Berlin Heidelberg. 
[42] G. Huang, G.-B. Huang, S. Song, and K. You, "Trends in extreme learning machines: A review," Neural Networks, vol. 61, pp. 32-48, 2015.

[43] A. Gaier and D. Ha, "Weight agnostic neural networks," arXiv preprint arXiv:1906.04358, 2019.

[44] J. Schmidhuber, D. Wierstra, M. Gagliolo, and F. Gomez, "Training recurrent networks by evolino," Neural Computation, vol. 19, no. 3, pp. 757-779, 2007.

[45] F. M. Bianchi, S. Scardapane, S. Løkse, and R. Jenssen, "Reservoir computing approaches for representation and classification of multivariate time series," arXiv preprint arXiv:1803.07870, 2018.

[46] N. Schaetti, M. Salomon, and R. Couturier, "Echo state networks-based reservoir computing for mnist handwritten digits recognition," in 2016 IEEE Intl Conference on Computational Science and Engineering (CSE) and IEEE Intl Conference on Embedded and Ubiquitous Computing (EUC) and 15th Intl Symposium on Distributed Computing and Applications for Business Engineering (DCABES), pp. 484-491, IEEE, 2016.

[47] M. Lukoševičius, "A practical guide to applying echo state networks," in Neural Networks: Tricks of the Trade, pp. 659-686, Springer, 2012.

[48] G. H. Golub, P. C. Hansen, and D. P. O'Leary, "Tikhonov regularization and total least squares," SIAM Journal on Matrix Analysis and Applications, vol. 21, no. 1, pp. 185-194, 1999.

[49] T. S. Furey, N. Cristianini, N. Duffy, D. W. Bednarski, M. Schummer, and D. Haussler, "Support vector machine classification and validation of cancer 
tissue samples using microarray expression data," Bioinformatics, vol. 16, no. 10, pp. 906-914, 2000.

[50] A. Rodan and P. Tino, "Minimum complexity echo state network," IEEE transactions on neural networks, vol. 22, no. 1, pp. 131-144, 2010.

[51] K. A. Johnson and R. S. Goody, "The original michaelis constant: translation of the 1913 michaelis-menten paper," Biochemistry, vol. 50, no. 39, pp. 8264-8269, 2011.

[52] C. V. Rao and A. P. Arkin, "Stochastic chemical kinetics and the quasi-steadystate assumption: Application to the gillespie algorithm," The Journal of chemical physics, vol. 118, no. 11, pp. 4999-5010, 2003.

[53] D. Y. Zhang and G. Seelig, "Dynamic dna nanotechnology using stranddisplacement reactions," Nature Chemistry, vol. 3, no. 2, p. 103, 2011.

[54] F. C. Simmel, B. Yurke, and H. R. Singh, "Principles and applications of nucleic acid strand displacement reactions," Chemical Reviews, vol. 119, no. 10, pp. 6326-6369, 2019.

[55] A. Fiannaca, L. La Paglia, M. La Rosa, G. Renda, R. Rizzo, S. Gaglio, A. Urso, et al., "Deep learning models for bacteria taxonomic classification of metagenomic data," BMC bioinformatics, vol. 19, no. 7, p. 198, 2018.

[56] C. E. Shannon, "A mathematical theory of communication," ACM SIGMOBILE mobile computing and communications review, vol. 5, no. 1, pp. 3-55, 2001.

[57] A. Goudarzi, P. Banda, M. R. Lakin, C. Teuscher, and D. Stefanovic, "A comparative study of reservoir computing for temporal signal processing," arXiv preprint arXiv:1401.2224, 2014. 
[58] C. Gallicchio and A. Micheli, "Architectural and markovian factors of echo state networks," Neural Networks, vol. 24, no. 5, pp. 440-456, 2011.

[59] Y. LeCun, L. Bottou, Y. Bengio, P. Haffner, et al., "Gradient-based learning applied to document recognition," Proceedings of the IEEE, vol. 86, no. 11, pp. 2278-2324, 1998.

[60] L. Wan, M. Zeiler, S. Zhang, Y. Le Cun, and R. Fergus, "Regularization of neural networks using dropconnect," in International Conference on Machine Learning, pp. 1058-1066, 2013.

[61] D. Dua and C. Graff, "UCI machine learning repository." University of California, Irvine, School of Information and Computer Sciences http://archive. ics.uci.edu/ml, 2017.

[62] N. G. Nguyen, V. A. Tran, D. L. Ngo, D. Phan, F. R. Lumbanraja, M. R. Faisal, B. Abapihi, M. Kubo, and K. Satou, "DNA sequence classification by convolutional neural network," Journal of Biomedical Science and Engineering, vol. 9, no. 05 , p. 280, 2016.

[63] R. J. Kuhn, W. Zhang, M. G. Rossmann, S. V. Pletnev, J. Corver, E. Lenches, C. T. Jones, S. Mukhopadhyay, P. R. Chipman, E. G. Strauss, et al., "Structure of dengue virus: implications for flavivirus organization, maturation, and fusion," Cell, vol. 108, no. 5, pp. 717-725, 2002.

[64] World Health Organization: Dengue and Severe Dengue, https://www.who. int/news-room/fact-sheets/detail/dengue-and-severe-dengue, Accessed: 11-15-19. 
[65] M. Gao, D. Daniel, H. Zou, S. Jiang, S. Lin, C. Huang, S. M. Hecht, and S. Chen, "Rapid detection of a dengue virus RNA sequence with single molecule sensitivity using tandem toehold-mediated displacement reactions," Chemical communications, vol. 54, no. 8, pp. 968-971, 2018.

[66] S. Gago, S. F. Elena, R. Flores, and R. Sanjuán, "Extremely high mutation rate of a hammerhead viroid," Science, vol. 323, no. 5919, pp. 1308-1308, 2009.

[67] P. Parameswaran, P. Charlebois, Y. Tellez, A. Nunez, E. M. Ryan, C. M. Malboeuf, J. Z. Levin, N. J. Lennon, A. Balmaseda, E. Harris, and M. R. Henn, "Genome-Wide Patterns of Intrahuman Dengue Virus Diversity Reveal Associations with Viral Phylogenetic Clade and Interhost Diversity," Journal of Virology, vol. 86, pp. 8546-8558, Aug. 2012.

[68] P. Mekha, K. Osathanunkul, and N. Teeyasuksaet, "Comparison of gene classification methods for dengue virus type based on codon usage," FEU Academic Review, vol. 11, pp. 7-7, 2017.

[69] S. Solis-Reyes, M. Avino, A. Poon, and L. Kari, "An open-source k-mer based machine learning tool for fast and accurate subtyping of HIV-1 genomes," PloS one, vol. 13, no. 11, p. e0206409, 2018.

[70] Virus Pathogen Resource: Dengue Virus, https://www.viprbrc.org/brc/ home.spg?decorator=flavi_dengue, Accessed: 03-15-19.

[71] F. Dubosson, J.-E. Ranvier, S. Bromuri, J.-P. Calbimonte, J. Ruiz, and M. Schumacher, "The open D1NAMO dataset: A multi-modal dataset for research on non-invasive type 1 diabetes management," Informatics in Medicine Unlocked, vol. 13, pp. 92-100, 2018. 
[72] G. Sparacino, F. Zanderigo, S. Corazza, A. Maran, A. Facchinetti, and C. Cobelli, "Glucose concentration can be predicted ahead in time from continuous glucose monitoring sensor time-series," IEEE Transactions on Biomedical Engineering, vol. 54, no. 5, pp. 931-937, 2007.

[73] A. Busia, G. E. Dahl, C. Fannjiang, D. H. Alexander, E. Dorfman, R. Poplin, C. Y. McLean, P.-C. Chang, and M. DePristo, "A deep learning approach to pattern recognition for short DNA sequences," bioRxiv, p. 353474, 2019.

[74] R. Rizzo, A. Fiannaca, M. La Rosa, and A. Urso, "A deep learning approach to DNA sequence classification," in International Meeting on Computational Intelligence Methods for Bioinformatics and Biostatistics, pp. 129-140, Springer, 2015.

[75] C. Angermueller, T. Pärnamaa, L. Parts, and O. Stegle, "Deep learning for computational biology," Molecular Systems Biology, vol. 12, no. 7, 2016.

[76] N. Schaetti, "Echotorch: Reservoir computing with PyTorch." https://github . com/nschaetti/EchoTorch, 2018.

[77] D. P. Kingma and J. Ba, "Adam: A method for stochastic optimization," arXiv preprint arXiv:1412.6980, 2014.

[78] M. C. Ozturk, D. Xu, and J. C. Prncipe, "Analysis and design of echo state networks," Neural Computation, vol. 19, no. 1, pp. 111-138, 2007.

[79] T. Mikolov, K. Chen, G. Corrado, and J. Dean, "Efficient estimation of word representations in vector space," arXiv preprint arXiv:1301.3781, 2013. 
[80] H. Dai, R. Umarov, H. Kuwahara, Y. Li, L. Song, and X. Gao, "Sequence2vec: a novel embedding approach for modeling transcription factor binding affinity landscape," Bioinformatics, vol. 33, no. 22, pp. 3575-3583, 2017.

[81] C. W. Brown, M. R. Lakin, A. FabryWood, E. K. Horwitz, N. A. Baker, D. Stefanovic, and S. W. Graves, "A Unified Sensor Architecture for Isothermal Detection of DoubleStranded DNA, Oligonucleotides, and Small Molecules," ChemBioChem, vol. 16, no. 5, pp. 725-730, 2015.

[82] J. Farfel and D. Stefanovic, "Towards practical biomolecular computers using microfluidic deoxyribozyme logic gate networks," in International Workshop on DNA-Based Computers, pp. 38-54, Springer, 2005.

[83] J. N. Zadeh, C. D. Steenberg, J. S. Bois, B. R. Wolfe, M. B. Pierce, A. R. Khan, R. M. Dirks, and N. A. Pierce, "NUPACK: Analysis and design of nucleic acid systems," Journal of Computational Chemistry, vol. 32, pp. 170-173, jan 2011.

[84] F. Li, H. Zhang, C. Lai, X.-F. Li, and X. C. Le, "A molecular translator that acts by binding-induced dna strand displacement for a homogeneous protein assay," Angewandte Chemie International Edition, vol. 51, no. 37, pp. 9317-9320. 


\section{Appendix: Tables of Results}

\begin{tabular}{|c|c|c|c|c|c|}
\hline Sparsity: & $\mathbf{0 . 1}$ & $\mathbf{0 . 2 5}$ & $\mathbf{0 . 5}$ & $\mathbf{0 . 7 5}$ & $\mathbf{1}$ \\
\hline Nodes & Mean \pm St. Dev. & Mean \pm St. Dev. & Mean \pm St. Dev. & Mean \pm St. Dev. & Mean \pm St. Dev. \\
\hline $\mathbf{1}$ & $5.04 \mathrm{E}-01 \pm 4.17 \mathrm{E}-02$ & $5.23 \mathrm{E}-01 \pm 7.00 \mathrm{E}-06$ & $4.22 \mathrm{E}-01 \pm 1.02 \mathrm{E}-01$ & $4.41 \mathrm{E}-01 \pm 1.12 \mathrm{E}-01$ & $3.40 \mathrm{E}-01 \pm 5.05 \mathrm{E}-02$ \\
\hline $\mathbf{2}$ & $4.73 \mathrm{E}-01 \pm 1.05 \mathrm{E}-01$ & $4.86 \mathrm{E}-01 \pm 1.24 \mathrm{E}-01$ & $5.72 \mathrm{E}-01 \pm 2.67 \mathrm{E}-01$ & $4.70 \mathrm{E}-01 \pm 1.36 \mathrm{E}-01$ & $6.74 \mathrm{E}-01 \pm 4.92 \mathrm{E}-01$ \\
\hline $\mathbf{3}$ & $4.37 \mathrm{E}-01 \pm 1.59 \mathrm{E}-01$ & $4.56 \mathrm{E}-01 \pm 1.54 \mathrm{E}-01$ & $5.10 \mathrm{E}-01 \pm 1.75 \mathrm{E}-01$ & $3.91 \mathrm{E}-01 \pm 1.51 \mathrm{E}-01$ & $4.81 \mathrm{E}-01 \pm 2.13 \mathrm{E}-01$ \\
\hline $\mathbf{5}$ & $7.13 \mathrm{E}-02 \pm 1.11 \mathrm{E}-01$ & $2.57 \mathrm{E}-02 \pm 5.19 \mathrm{E}-02$ & $1.13 \mathrm{E}-01 \pm 1.17 \mathrm{E}-01$ & $9.20 \mathrm{E}-02 \pm 1.03 \mathrm{E}-01$ & $1.13 \mathrm{E}-01 \pm 5.45 \mathrm{E}-02$ \\
\hline $\mathbf{1 0}$ & $6.15 \mathrm{E}-05 \pm 1.28 \mathrm{E}-04$ & $2.79 \mathrm{E}-03 \pm 3.46 \mathrm{E}-03$ & $1.17 \mathrm{E}-03 \pm 1.78 \mathrm{E}-03$ & $6.29 \mathrm{E}-04 \pm 8.99 \mathrm{E}-04$ & $3.22 \mathrm{E}-03 \pm 4.96 \mathrm{E}-03$ \\
\hline $\mathbf{2 0}$ & $5.63 \mathrm{E}-06 \pm 4.84 \mathrm{E}-06$ & $2.84 \mathrm{E}-05 \pm 1.96 \mathrm{E}-05$ & $3.04 \mathrm{E}-05 \pm 2.22 \mathrm{E}-05$ & $5.90 \mathrm{E}-05 \pm 4.67 \mathrm{E}-05$ & $2.45 \mathrm{E}-04 \pm 3.78 \mathrm{E}-04$ \\
\hline $\mathbf{3 0}$ & $1.15 \mathrm{E}-05 \pm 8.61 \mathrm{E}-06$ & $8.63 \mathrm{E}-06 \pm 6.24 \mathrm{E}-06$ & $2.50 \mathrm{E}-05 \pm 1.44 \mathrm{E}-05$ & $2.98 \mathrm{E}-05 \pm 4.91 \mathrm{E}-05$ & $3.96 \mathrm{E}-05 \pm 3.54 \mathrm{E}-05$ \\
\hline $\mathbf{5 0}$ & $1.06 \mathrm{E}-05 \pm 7.87 \mathrm{E}-06$ & $8.58 \mathrm{E}-06 \pm 6.33 \mathrm{E}-06$ & $1.35 \mathrm{E}-05 \pm 4.31 \mathrm{E}-06$ & $5.35 \mathrm{E}-06 \pm 2.39 \mathrm{E}-06$ & $8.28 \mathrm{E}-06 \pm 5.54 \mathrm{E}-06$ \\
\hline $\mathbf{1 0 0}$ & $7.04 \mathrm{E}-06 \pm 2.78 \mathrm{E}-06$ & $6.94 \mathrm{E}-06 \pm 3.85 \mathrm{E}-06$ & $6.12 \mathrm{E}-06 \pm 3.30 \mathrm{E}-06$ & $8.12 \mathrm{E}-06 \pm 3.48 \mathrm{E}-06$ & $6.26 \mathrm{E}-06 \pm 2.66 \mathrm{E}-06$ \\
\hline $\mathbf{2 0 0}$ & $6.86 \mathrm{E}-06 \pm 3.02 \mathrm{E}-06$ & $8.89 \mathrm{E}-06 \pm 5.10 \mathrm{E}-06$ & $8.04 \mathrm{E}-06 \pm 3.81 \mathrm{E}-06$ & $7.80 \mathrm{E}-06 \pm 3.56 \mathrm{E}-06$ & $8.61 \mathrm{E}-06 \pm 4.82 \mathrm{E}-06$ \\
\hline $\mathbf{3 0 0}$ & $1.04 \mathrm{E}-05 \pm 5.05 \mathrm{E}-06$ & $1.24 \mathrm{E}-05 \pm 6.14 \mathrm{E}-06$ & $9.78 \mathrm{E}-06 \pm 4.60 \mathrm{E}-06$ & $1.27 \mathrm{E}-05 \pm 6.40 \mathrm{E}-06$ & $1.07 \mathrm{E}-05 \pm 5.07 \mathrm{E}-06$ \\
\hline $\mathbf{5 0 0}$ & $2.14 \mathrm{E}-05 \pm 1.02 \mathrm{E}-05$ & $1.84 \mathrm{E}-05 \pm 8.35 \mathrm{E}-06$ & $2.04 \mathrm{E}-05 \pm 9.37 \mathrm{E}-06$ & $1.99 \mathrm{E}-05 \pm 9.20 \mathrm{E}-06$ & $1.95 \mathrm{E}-05 \pm 8.95 \mathrm{E}-06$ \\
\hline
\end{tabular}

Figure 1: Table with the mean MSE \pm the standard deviation from the NARMA-A with ESN results.

\begin{tabular}{|c|c|c|c|c|c|c|c|}
\hline Spectral Radius: & $\mathbf{0 . 1}$ & $\mathbf{0 . 3}$ & $\mathbf{0 . 5}$ & $\mathbf{0 . 7}$ & $\mathbf{0 . 9}$ & $\mathbf{0 . 9 5}$ & $\mathbf{0 . 9 9}$ \\
\hline Nodes & Mean \pm St. Dev. & Mean \pm St. Dev. & Mean \pm St. Dev. & Mean \pm St. Dev. & Mean \pm St. Dev. & Mean \pm St. Dev. & Mean \pm St. Dev. \\
\hline 6 & $5.15 \mathrm{E}-07 \pm 6.18 \mathrm{E}-07$ & $2.75 \mathrm{E}-06 \pm 2.45 \mathrm{E}-06$ & $7.16 \mathrm{E}-06 \pm 6.81 \mathrm{E}-06$ & $1.22 \mathrm{E}-04 \pm 6.92 \mathrm{E}-06$ & $3.12 \mathrm{E}-03 \pm 1.34 \mathrm{E}-04$ & $6.38 \mathrm{E}-03 \pm 2.77 \mathrm{E}-04$ & $1.14 \mathrm{E}-02 \pm 3.84 \mathrm{E}-04$ \\
\hline 10 & $4.11 \mathrm{E}-09 \pm 4.75 \mathrm{E}-09$ & $1.15 \mathrm{E}-07 \pm 7.12 \mathrm{E}-08$ & $9.44 \mathrm{E}-07 \pm 1.33 \mathrm{E}-06$ & $4.66 \mathrm{E}-06 \pm 3.67 \mathrm{E}-06$ & $5.36 \mathrm{E}-05 \pm 5.09 \mathrm{E}-06$ & $1.59 \mathrm{E}-04 \pm 1.32 \mathrm{E}-05$ & $4.10 \mathrm{E}-04 \pm 5.48 \mathrm{E}-05$ \\
\hline 14 & $3.77 \mathrm{E}-10 \pm 3.04 \mathrm{E}-10$ & $1.27 \mathrm{E}-08 \pm 8.77 \mathrm{E}-09$ & $1.07 \mathrm{E}-07 \pm 2.68 \mathrm{E}-08$ & $9.56 \mathrm{E}-07 \pm 6.72 \mathrm{E}-07$ & $9.69 \mathrm{E}-06 \pm 3.75 \mathrm{E}-06$ & $2.80 \mathrm{E}-05 \pm 1.25 \mathrm{E}-05$ & $1.02 \mathrm{E}-04 \pm 4.15 \mathrm{E}-05$ \\
\hline 18 & $1.11 \mathrm{E}-10 \pm 1.13 \mathrm{E}-10$ & $8.13 \mathrm{E}-10 \pm 4.74 \mathrm{E}-10$ & $1.89 \mathrm{E}-08 \pm 1.05 \mathrm{E}-08$ & $3.93 \mathrm{E}-07 \pm 3.37 \mathrm{E}-07$ & $9.05 \mathrm{E}-06 \pm 6.87 \mathrm{E}-06$ & $2.79 \mathrm{E}-05 \pm 2.41 \mathrm{E}-05$ & $7.75 \mathrm{E}-05 \pm 5.42 \mathrm{E}-05$ \\
\hline 30 & $1.98 \mathrm{E}-11 \pm 1.35 \mathrm{E}-11$ & $3.54 \mathrm{E}-11 \pm 1.56 \mathrm{E}-11$ & $1.13 \mathrm{E}-09 \pm 5.79 \mathrm{E}-10$ & $3.89 \mathrm{E}-08 \pm 1.10 \mathrm{E}-08$ & $8.39 \mathrm{E}-07 \pm 3.45 \mathrm{E}-07$ & $3.36 \mathrm{E}-06 \pm 1.82 \mathrm{E}-06$ & $1.44 \mathrm{E}-05 \pm 1.74 \mathrm{E}-06$ \\
\hline 42 & $8.49 \mathrm{E}-12 \pm 6.07 \mathrm{E}-12$ & $1.10 \mathrm{E}-11 \pm 7.02 \mathrm{E}-12$ & $2.31 \mathrm{E}-10 \pm 1.45 \mathrm{E}-10$ & $6.15 \mathrm{E}-09 \pm 1.65 \mathrm{E}-09$ & $2.83 \mathrm{E}-07 \pm 1.20 \mathrm{E}-07$ & $9.50 \mathrm{E}-07 \pm 3.09 \mathrm{E}-07$ & $4.68 \mathrm{E}-06 \pm 6.31 \mathrm{E}-07$ \\
\hline 62 & $3.41 \mathrm{E}-12 \pm 7.76 \mathrm{E}-13$ & $2.65 \mathrm{E}-12 \pm 1.12 \mathrm{E}-12$ & $2.59 \mathrm{E}-11 \pm 1.58 \mathrm{E}-11$ & $1.03 \mathrm{E}-09 \pm 5.31 \mathrm{E}-10$ & $1.31 \mathrm{E}-07 \pm 9.60 \mathrm{E}-08$ & $4.62 \mathrm{E}-07 \pm 3.90 \mathrm{E}-07$ & $2.41 \mathrm{E}-06 \pm 8.19 \mathrm{E}-07$ \\
\hline 102 & $1.80 \mathrm{E}-12 \pm 5.12 \mathrm{E}-13$ & $8.98 \mathrm{E}-13 \pm 3.58 \mathrm{E}-13$ & $2.07 \mathrm{E}-12 \pm 8.54 \mathrm{E}-13$ & $1.10 \mathrm{E}-10 \pm 5.38 \mathrm{E}-11$ & $1.91 \mathrm{E}-08 \pm 9.31 \mathrm{E}-09$ & $9.93 \mathrm{E}-08 \pm 6.07 \mathrm{E}-08$ & $7.06 \mathrm{E}-07 \pm 2.26 \mathrm{E}-07$ \\
\hline 162 & $1.23 \mathrm{E}-12 \pm 3.06 \mathrm{E}-13$ & $3.76 \mathrm{E}-13 \pm 6.09 \mathrm{E}-14$ & $4.80 \mathrm{E}-13 \pm 1.06 \mathrm{E}-13$ & $8.31 \mathrm{E}-12 \pm 3.68 \mathrm{E}-12$ & $4.53 \mathrm{E}-09 \pm 1.10 \mathrm{E}-09$ & $3.18 \mathrm{E}-08 \pm 9.78 \mathrm{E}-09$ & $3.27 \mathrm{E}-07 \pm 1.32 \mathrm{E}-07$ \\
\hline
\end{tabular}

Figure 2: Table with the mean MSE \pm the standard deviation from the NARMA-A with oscillating neural network results. 


\begin{tabular}{|c|c|c|c|c|c|}
\hline Sparsity: & $\mathbf{0 . 1}$ & $\mathbf{0 . 2 5}$ & $\mathbf{0 . 5}$ & $\mathbf{0 . 7 5}$ & $\mathbf{1}$ \\
\hline Nodes & Mean \pm St. Dev. & Mean \pm St. Dev. & Mean \pm St. Dev. & Mean \pm St. Dev. & Mean \pm St. Dev. \\
\hline 1 & $9.09 \mathrm{E}-03 \pm 1.25 \mathrm{E}-03$ & $9.79 \mathrm{E}-03 \pm 2.23 \mathrm{E}-03$ & $9.39 \mathrm{E}-03 \pm 2.66 \mathrm{E}-03$ & $9.76 \mathrm{E}-03 \pm 2.29 \mathrm{E}-03$ & $8.84 \mathrm{E}-03 \pm 2.62 \mathrm{E}-03$ \\
\hline 2 & $8.13 \mathrm{E}-03 \pm 3.64 \mathrm{E}-04$ & $7.66 \mathrm{E}-03 \pm 1.19 \mathrm{E}-03$ & $7.87 \mathrm{E}-03 \pm 9.55 \mathrm{E}-04$ & $6.80 \mathrm{E}-03 \pm 9.75 \mathrm{E}-04$ & $9.33 \mathrm{E}-03 \pm 1.80 \mathrm{E}-03$ \\
\hline 3 & $7.37 \mathrm{E}-03 \pm 1.32 \mathrm{E}-03$ & $7.16 \mathrm{E}-03 \pm 1.24 \mathrm{E}-03$ & $7.32 \mathrm{E}-03 \pm 1.09 \mathrm{E}-03$ & $7.09 \mathrm{E}-03 \pm 1.22 \mathrm{E}-03$ & $7.16 \mathrm{E}-03 \pm 1.31 \mathrm{E}-03$ \\
\hline 5 & $7.62 \mathrm{E}-03 \pm 8.11 \mathrm{E}-04$ & $7.08 \mathrm{E}-03 \pm 9.04 \mathrm{E}-04$ & $6.77 \mathrm{E}-03 \pm 9.34 \mathrm{E}-04$ & $6.84 \mathrm{E}-03 \pm 8.44 \mathrm{E}-04$ & $6.85 \mathrm{E}-03 \pm 1.11 \mathrm{E}-03$ \\
\hline 10 & $5.15 \mathrm{E}-03 \pm 8.21 \mathrm{E}-04$ & $5.15 \mathrm{E}-03 \pm 1.30 \mathrm{E}-03$ & $5.60 \mathrm{E}-03 \pm 1.30 \mathrm{E}-03$ & $5.26 \mathrm{E}-03 \pm 1.44 \mathrm{E}-03$ & $5.60 \mathrm{E}-03 \pm 1.09 \mathrm{E}-03$ \\
\hline 20 & $2.47 \mathrm{E}-03 \pm 4.56 \mathrm{E}-04$ & $2.31 \mathrm{E}-03 \pm 3.17 \mathrm{E}-04$ & $2.17 \mathrm{E}-03 \pm 2.62 \mathrm{E}-04$ & $2.18 \mathrm{E}-03 \pm 2.65 \mathrm{E}-04$ & $2.21 \mathrm{E}-03 \pm 5.14 \mathrm{E}-04$ \\
\hline 30 & $2.01 \mathrm{E}-03 \pm 9.81 \mathrm{E}-05$ & $1.97 \mathrm{E}-03 \pm 1.60 \mathrm{E}-04$ & $1.81 \mathrm{E}-03 \pm 8.73 \mathrm{E}-05$ & $1.87 \mathrm{E}-03 \pm 1.08 \mathrm{E}-04$ & $1.86 \mathrm{E}-03 \pm 1.24 \mathrm{E}-04$ \\
\hline 40 & $1.81 \mathrm{E}-03 \pm 9.10 \mathrm{E}-05$ & $1.88 \mathrm{E}-03 \pm 1.45 \mathrm{E}-04$ & $1.84 \mathrm{E}-03 \pm 6.89 \mathrm{E}-05$ & $1.83 \mathrm{E}-03 \pm 6.52 \mathrm{E}-05$ & $1.83 \mathrm{E}-03 \pm 1.53 \mathrm{E}-04$ \\
\hline 50 & $1.78 \mathrm{E}-03 \pm 1.25 \mathrm{E}-04$ & $1.78 \mathrm{E}-03 \pm 7.99 \mathrm{E}-05$ & $1.71 \mathrm{E}-03 \pm 1.22 \mathrm{E}-04$ & $1.70 \mathrm{E}-03 \pm 1.03 \mathrm{E}-04$ & $1.70 \mathrm{E}-03 \pm 1.40 \mathrm{E}-04$ \\
\hline 100 & $1.38 \mathrm{E}-03 \pm 1.05 \mathrm{E}-04$ & $1.37 \mathrm{E}-03 \pm 1.20 \mathrm{E}-04$ & $1.30 \mathrm{E}-03 \pm 1.90 \mathrm{E}-04$ & $1.23 \mathrm{E}-03 \pm 1.56 \mathrm{E}-04$ & $1.42 \mathrm{E}-03 \pm 1.46 \mathrm{E}-04$ \\
\hline 200 & $8.68 \mathrm{E}-04 \pm 4.93 \mathrm{E}-05$ & $7.75 \mathrm{E}-04 \pm 1.71 \mathrm{E}-04$ & $8.07 \mathrm{E}-04 \pm 1.64 \mathrm{E}-04$ & $8.36 \mathrm{E}-04 \pm 9.52 \mathrm{E}-05$ & $7.63 \mathrm{E}-04 \pm 1.09 \mathrm{E}-04$ \\
\hline 300 & $6.52 \mathrm{E}-04 \pm 1.17 \mathrm{E}-04$ & $5.16 \mathrm{E}-04 \pm 1.31 \mathrm{E}-04$ & $6.88 \mathrm{E}-04 \pm 6.18 \mathrm{E}-05$ & $5.55 \mathrm{E}-04 \pm 8.96 \mathrm{E}-05$ & $6.17 \mathrm{E}-04 \pm 1.48 \mathrm{E}-04$ \\
\hline 500 & $4.10 \mathrm{E}-04 \pm 1.29 \mathrm{E}-04$ & $3.96 \mathrm{E}-04 \pm 4.77 \mathrm{E}-05$ & $3.89 \mathrm{E}-04 \pm 4.48 \mathrm{E}-05$ & $3.84 \mathrm{E}-04 \pm 5.41 \mathrm{E}-05$ & $3.84 \mathrm{E}-04 \pm 8.92 \mathrm{E}-05$ \\
\hline 1000 & $2.94 \mathrm{E}-04 \pm 4.56 \mathrm{E}-05$ & $2.54 \mathrm{E}-04 \pm 5.45 \mathrm{E}-05$ & $2.69 \mathrm{E}-04 \pm 4.88 \mathrm{E}-05$ & $2.41 \mathrm{E}-04 \pm 4.74 \mathrm{E}-05$ & $2.53 \mathrm{E}-04 \pm 6.11 \mathrm{E}-05$ \\
\hline 1500 & $2.06 \mathrm{E}-04 \pm 3.56 \mathrm{E}-05$ & $1.83 \mathrm{E}-04 \pm 1.34 \mathrm{E}-05$ & $1.91 \mathrm{E}-04 \pm 2.60 \mathrm{E}-05$ & $2.00 \mathrm{E}-04 \pm 2.32 \mathrm{E}-05$ & $1.93 \mathrm{E}-04 \pm 2.10 \mathrm{E}-05$ \\
\hline 2000 & $1.80 \mathrm{E}-04 \pm 1.74 \mathrm{E}-05$ & $1.90 \mathrm{E}-04 \pm 2.27 \mathrm{E}-05$ & $1.92 \mathrm{E}-04 \pm 3.11 \mathrm{E}-05$ & $1.93 \mathrm{E}-04 \pm 2.44 \mathrm{E}-05$ & $1.84 \mathrm{E}-04 \pm 1.83 \mathrm{E}-05$ \\
\hline
\end{tabular}

Figure 3: Table with the mean MSE \pm the standard deviation from the NARMA-10 with ESN results.

\begin{tabular}{|c|c|c|c|c|c|c|c|}
\hline Spectral Radius: & 0.1 & 0.3 & 0.5 & 0.7 & 0.9 & 0.95 & 0.99 \\
\hline Nodes & Mean \pm St. Dev. & Mean \pm St. Dev. & Mean \pm St. Dev. & Mean \pm St. Dev. & Mean \pm St. Dev. & Mean \pm St. Dev. & Mean \pm St. Dev. \\
\hline 6 & $9.10 \mathrm{E}-03 \pm 4.70 \mathrm{E}-05$ & $8.44 \mathrm{E}-03 \pm 4.84 \mathrm{E}-04$ & $8.07 \mathrm{E}-03 \pm 5.22 \mathrm{E}-04$ & $7.21 \mathrm{E}-03 \pm 4.81 \mathrm{E}-04$ & $6.69 \mathrm{E}-03 \pm 5.60 \mathrm{E}-04$ & $6.79 \mathrm{E}-03 \pm 1.95 \mathrm{E}-05$ & $8.55 \mathrm{E}-03 \pm 7.70 \mathrm{E}-04$ \\
\hline 10 & $9.01 \mathrm{E}-03 \pm 3.18 \mathrm{E}-05$ & $7.74 \mathrm{E}-03 \pm 5.27 \mathrm{E}-04$ & $3.36 \mathrm{E}-03 \pm 2.89 \mathrm{E}-04$ & $3.42 \mathrm{E}-03 \pm 3.06 \mathrm{E}-04$ & $4.20 \mathrm{E}-03 \pm 3.14 \mathrm{E}-04$ & $4.70 \mathrm{E}-03 \pm 4.79 \mathrm{E}-05$ & $6.76 \mathrm{E}-03 \pm 5.77 \mathrm{E}-04$ \\
\hline 14 & $9.04 \mathrm{E}-03 \pm 3.81 \mathrm{E}-05$ & $7.23 \mathrm{E}-03 \pm 6.16 \mathrm{E}-04$ & $3.31 \mathrm{E}-03 \pm 2.85 \mathrm{E}-04$ & $2.90 \mathrm{E}-03 \pm 4.15 \mathrm{E}-04$ & $2.71 \mathrm{E}-03 \pm 4.56 \mathrm{E}-04$ & $2.79 \mathrm{E}-03 \pm 5.55 \mathrm{E}-04$ & $4.43 \mathrm{E}-03 \pm 7.68 \mathrm{E}-04$ \\
\hline 18 & $9.06 \mathrm{E}-03 \pm 9.57 \mathrm{E}-07$ & $6.24 \mathrm{E}-03 \pm 5.39 \mathrm{E}-04$ & $3.37 \mathrm{E}-03 \pm 2.98 \mathrm{E}-04$ & $2.53 \mathrm{E}-03 \pm 2.95 \mathrm{E}-04$ & $2.55 \mathrm{E}-03 \pm 2.53 \mathrm{E}-04$ & $2.36 \mathrm{E}-03 \pm 8.00 \mathrm{E}-05$ & $3.27 \mathrm{E}-03 \pm 3.34 \mathrm{E}-04$ \\
\hline 30 & $9.06 \mathrm{E}-03 \pm 1.72 \mathrm{E}-06$ & $4.79 \mathrm{E}-03 \pm 4.58 \mathrm{E}-04$ & $2.50 \mathrm{E}-03 \pm 3.06 \mathrm{E}-04$ & $2.20 \mathrm{E}-03 \pm 2.89 \mathrm{E}-04$ & $2.09 \mathrm{E}-03 \pm 2.03 \mathrm{E}-04$ & $2.05 \mathrm{E}-03 \pm 1.39 \mathrm{E}-04$ & $2.61 \mathrm{E}-03 \pm 2.52 \mathrm{E}-04$ \\
\hline 42 & $9.05 \mathrm{E}-03 \pm 4.71 \mathrm{E}-06$ & $4.24 \mathrm{E}-03 \pm 4.13 \mathrm{E}-04$ & $2.34 \mathrm{E}-03 \pm 2.74 \mathrm{E}-04$ & $1.92 \mathrm{E}-03 \pm 1.88 \mathrm{E}-04$ & $1.95 \mathrm{E}-03 \pm 7.23 \mathrm{E}-05$ & $1.91 \mathrm{E}-03 \pm 5.10 \mathrm{E}-05$ & $2.56 \mathrm{E}-03 \pm 2.55 \mathrm{E}-04$ \\
\hline 62 & $9.05 \mathrm{E}-03 \pm 2.22 \mathrm{E}-06$ & $3.83 \mathrm{E}-03 \pm 3.79 \mathrm{E}-04$ & $2.19 \mathrm{E}-03 \pm 2.30 \mathrm{E}-04$ & $1.68 \mathrm{E}-03 \pm 7.66 \mathrm{E}-05$ & $1.53 \mathrm{E}-03 \pm 6.88 \mathrm{E}-05$ & $1.62 \mathrm{E}-03 \pm 5.97 \mathrm{E}-05$ & $2.46 \mathrm{E}-03 \pm 5.81 \mathrm{E}-04$ \\
\hline 102 & $9.04 \mathrm{E}-03 \pm 5.81 \mathrm{E}-07$ & $3.43 \mathrm{E}-03 \pm 3.63 \mathrm{E}-04$ & $2.11 \mathrm{E}-03 \pm 2.25 \mathrm{E}-04$ & $1.44 \mathrm{E}-03 \pm 1.24 \mathrm{E}-04$ & $1.43 \mathrm{E}-03 \pm 6.35 \mathrm{E}-05$ & $1.44 \mathrm{E}-03 \pm 4.65 \mathrm{E}-05$ & $2.48 \mathrm{E}-03 \pm 8.11 \mathrm{E}-04$ \\
\hline 162 & $9.03 \mathrm{E}-03 \pm 1.13 \mathrm{E}-06$ & $3.20 \mathrm{E}-03 \pm 3.34 \mathrm{E}-04$ & $2.02 \mathrm{E}-03 \pm 1.99 \mathrm{E}-04$ & $1.29 \mathrm{E}-03 \pm 7.86 \mathrm{E}-05$ & $1.38 \mathrm{E}-03 \pm 6.85 \mathrm{E}-05$ & $1.79 \mathrm{E}-03 \pm 5.98 \mathrm{E}-04$ & $3.50 \mathrm{E}-03 \pm 6.66 \mathrm{E}-04$ \\
\hline 322 & $9.02 \mathrm{E}-03 \pm 1.38 \mathrm{E}-06$ & $3.25 \mathrm{E}-03 \pm 2.48 \mathrm{E}-05$ & $2.05 \mathrm{E}-03 \pm 6.10 \mathrm{E}-05$ & $1.21 \mathrm{E}-03 \pm 2.02 \mathrm{E}-05$ & $1.30 \mathrm{E}-03 \pm 7.72 \mathrm{E}-05$ & $2.76 \mathrm{E}-03 \pm 1.95 \mathrm{E}-05$ & $3.51 \mathrm{E}-03 \pm 1.12 \mathrm{E}-03$ \\
\hline 502 & $9.01 \mathrm{E}-03 \pm 1.85 \mathrm{E}-06$ & $3.18 \mathrm{E}-03 \pm 1.67 \mathrm{E}-06$ & $1.95 \mathrm{E}-03 \pm 2.47 \mathrm{E}-05$ & $1.14 \mathrm{E}-03 \pm 1.91 \mathrm{E}-05$ & $1.29 \mathrm{E}-03 \pm 7.80 \mathrm{E}-06$ & $2.48 \mathrm{E}-03 \pm 3.16 \mathrm{E}-04$ & $4.12 \mathrm{E}-03 \pm 7.61 \mathrm{E}-06$ \\
\hline 1002 & $8.99 \mathrm{E}-03 \pm 1.01 \mathrm{E}-06$ & $3.11 \mathrm{E}-03 \pm 5.35 \mathrm{E}-06$ & $1.86 \mathrm{E}-03 \pm 3.07 \mathrm{E}-05$ & $1.09 \mathrm{E}-03 \pm 2.79 \mathrm{E}-05$ & $1.25 \mathrm{E}-03 \pm 1.99 \mathrm{E}-05$ & $2.57 \mathrm{E}-03 \pm 1.56 \mathrm{E}-05$ & $3.35 \mathrm{E}-03 \pm 8.19 \mathrm{E}-04$ \\
\hline
\end{tabular}

Figure 4: Table with the mean MSE \pm the standard deviation from the NARMA-10 with oscillating neural network results. 


\begin{tabular}{|c|l|l|l|l|l|}
\hline Sparsity: & \multicolumn{1}{|c|}{0.1} & \multicolumn{1}{c|}{0.25} & \multicolumn{1}{c|}{0.5} & \multicolumn{1}{c|}{0.75} & 1 \\
\hline Nodes & Mean \pm St. Dev. & Mean \pm St. Dev. & Mean \pm St. Dev. & Mean \pm St. Dev. & Mean \pm St. Dev. \\
\hline 1 & $0.222 \pm 0.215$ & $0.210 \pm 0.194$ & $0.359 \pm 0.233$ & $0.476 \pm 0.047$ & $0.477 \pm 0.049$ \\
\hline 2 & $0.248 \pm 0.261$ & $0.262 \pm 0.284$ & $0.481 \pm 0.332$ & $0.694 \pm 0.033$ & $0.664 \pm 0.040$ \\
\hline 3 & $0.284 \pm 0.322$ & $0.543 \pm 0.385$ & $0.769 \pm 0.008$ & $0.778 \pm 0.007$ & $0.765 \pm 0.023$ \\
\hline 5 & $0.339 \pm 0.418$ & $0.807 \pm 0.023$ & $0.814 \pm 0.017$ & $0.814 \pm 0.010$ & $0.819 \pm 0.004$ \\
\hline 10 & $0.630 \pm 0.412$ & $0.901 \pm 0.009$ & $0.899 \pm 0.010$ & $0.903 \pm 0.011$ & $0.900 \pm 0.013$ \\
\hline 20 & $0.918 \pm 0.000$ & $0.913 \pm 0.004$ & $0.913 \pm 0.003$ & $0.917 \pm 0.004$ & $0.915 \pm 0.004$ \\
\hline 30 & $0.931 \pm 0.003$ & $0.932 \pm 0.004$ & $0.934 \pm 0.003$ & $0.931 \pm 0.003$ & $0.932 \pm 0.001$ \\
\hline 40 & $0.939 \pm 0.003$ & $0.939 \pm 0.001$ & $0.939 \pm 0.001$ & $0.940 \pm 0.002$ & $0.936 \pm 0.004$ \\
\hline 50 & $0.947 \pm 0.002$ & $0.946 \pm 0.003$ & $0.946 \pm 0.003$ & $0.946 \pm 0.002$ & $0.946 \pm 0.003$ \\
\hline 100 & $0.954 \pm 0.000$ & $0.949 \pm 0.001$ & $0.952 \pm 0.002$ & $0.952 \pm 0.001$ & $0.951 \pm 0.001$ \\
\hline 200 & $0.960 \pm 0.001$ & $0.959 \pm 0.003$ & $0.958 \pm 0.005$ & $0.959 \pm 0.001$ & $0.959 \pm 0.000$ \\
\hline 300 & $0.958 \pm 0.004$ & $0.957 \pm 0.003$ & $0.957 \pm 0.001$ & $0.956 \pm 0.002$ & $0.958 \pm 0.000$ \\
\hline 500 & $0.957 \pm 0.001$ & $0.954 \pm 0.007$ & $0.956 \pm 0.004$ & $0.957 \pm 0.001$ & $0.955 \pm 0.003$ \\
\hline 1000 & $0.961 \pm 0.001$ & $0.955 \pm 0.004$ & $0.959 \pm 0.001$ & $0.959 \pm 0.001$ & $0.957 \pm 0.001$ \\
\hline
\end{tabular}

Figure 5: Table with the mean accuracy \pm the standard deviation from the MNIST with ESN results.

\begin{tabular}{|c|l|l|l|l|}
\hline Sparsity: & \multicolumn{1}{|c|}{0.1} & \multicolumn{1}{c|}{0.25} & \multicolumn{1}{c|}{0.5} & \multicolumn{1}{c|}{1} \\
\hline Nodes & Mean \pm St. Dev. & Mean \pm St. Dev. & Mean \pm St. Dev. & Mean \pm St. Dev. \\
\hline 1 & $0.098 \pm 0.000$ & $0.098 \pm 0.000$ & $0.098 \pm 0.000$ & $0.121 \pm 0.000$ \\
\hline 2 & $0.098 \pm 0.100$ & $0.098 \pm 0.250$ & $0.121 \pm 0.500$ & $0.129 \pm 1.000$ \\
\hline 3 & $0.098 \pm 0.000$ & $0.098 \pm 0.000$ & $0.166 \pm 0.000$ & $0.157 \pm 0.004$ \\
\hline 5 & $0.098 \pm 0.000$ & $0.120 \pm 0.000$ & $0.194 \pm 0.006$ & $0.185 \pm 0.012$ \\
\hline 10 & $0.352 \pm 0.000$ & $0.199 \pm 0.000$ & $0.186 \pm 0.004$ & $0.116 \pm 0.017$ \\
\hline 20 & $0.183 \pm 0.000$ & $0.160 \pm 0.095$ & $0.144 \pm 0.027$ & $0.151 \pm 0.039$ \\
\hline 30 & $0.218 \pm 0.016$ & $0.160 \pm 0.052$ & $0.168 \pm 0.031$ & $0.202 \pm 0.066$ \\
\hline 50 & $0.159 \pm 0.053$ & $0.191 \pm 0.110$ & $0.244 \pm 0.103$ & $0.266 \pm 0.111$ \\
\hline 100 & $0.190 \pm 0.079$ & $0.259 \pm 0.101$ & $0.358 \pm 0.111$ & $0.513 \pm 0.105$ \\
\hline 200 & $0.302 \pm 0.137$ & $0.476 \pm 0.129$ & $0.571 \pm 0.114$ & $0.597 \pm 0.082$ \\
\hline 300 & $0.445 \pm 0.156$ & $0.593 \pm 0.118$ & $0.631 \pm 0.070$ & $0.613 \pm 0.075$ \\
\hline 500 & $0.572 \pm 0.125$ & $0.681 \pm 0.062$ & $0.658 \pm 0.098$ & $0.600 \pm 0.140$ \\
\hline 1000 & $0.743 \pm 0.075$ & $0.699 \pm 0.078$ & $0.627 \pm 0.119$ & $0.579 \pm 0.112$ \\
\hline 2000 & $0.768 \pm 0.077$ & & & \\
\hline 4000 & $0.731 \pm 0.121$ & & & \\
\hline
\end{tabular}

Figure 6: Table with the mean accuracy \pm the standard deviation from the MNIST with ESN last reservoir output only results. 


\begin{tabular}{|c|c|c|c|c|c|}
\hline Spectral Radius: & 0.3 & 0.5 & 0.9 & 0.95 & 0.99 \\
\hline Nodes & Mean \pm St. Dev. & Mean \pm St. Dev. & Mean \pm St. Dev. & Mean \pm St. Dev. & Mean \pm St. Dev. \\
\hline 6 & $0.870 \pm 0.019$ & $0.870 \pm 0.020$ & $0.865 \pm 0.019$ & $0.862 \pm 0.020$ & $0.861 \pm 0.019$ \\
\hline 10 & $0.907 \pm 0.008$ & $0.905 \pm 0.008$ & $0.900 \pm 0.009$ & $0.899 \pm 0.009$ & $0.899 \pm 0.009$ \\
\hline 14 & $0.918 \pm 0.007$ & $0.918 \pm 0.007$ & $0.916 \pm 0.008$ & $0.915 \pm 0.008$ & $0.915 \pm 0.008$ \\
\hline 18 & $0.927 \pm 0.006$ & $0.926 \pm 0.005$ & $0.924 \pm 0.004$ & $0.923 \pm 0.005$ & $0.923 \pm 0.005$ \\
\hline 30 & $0.935 \pm 0.003$ & $0.935 \pm 0.005$ & $0.938 \pm 0.004$ & $0.940 \pm 0.003$ & $0.939 \pm 0.004$ \\
\hline 42 & $0.940 \pm 0.002$ & $0.942 \pm 0.000$ & $0.946 \pm 0.000$ & $0.946 \pm 0.002$ & $0.946 \pm 0.001$ \\
\hline 102 & $0.948 \pm 0.001$ & $0.952 \pm 0.001$ & $0.959 \pm 0.002$ & $0.960 \pm 0.002$ & $0.960 \pm 0.002$ \\
\hline 162 & $0.951 \pm 0.002$ & $0.955 \pm 0.001$ & $0.962 \pm 0.002$ & $0.965 \pm 0.002$ & $0.965 \pm 0.001$ \\
\hline 322 & $0.948 \pm 0.000$ & $0.953 \pm 0.002$ & $0.966 \pm 0.001$ & $0.966 \pm 0.001$ & $0.969 \pm 0.003$ \\
\hline 502 & $0.941 \pm 0.011$ & $0.943 \pm 0.013$ & $0.967 \pm 0.002$ & $0.966 \pm 0.002$ & $0.966 \pm 0.005$ \\
\hline
\end{tabular}

Figure 7: Table with the mean accuracy \pm the standard deviation from the MNIST with oscillating neural network results.

\begin{tabular}{|c|c|c|c|c|c|}
\hline Sparsity: & 0.1 & 0.25 & 0.5 & 0.75 & 1 \\
\hline Nodes & Mean \pm St. Dev. & Mean \pm St. Dev. & Mean \pm St. Dev. & Mean \pm St. Dev. & Mean \pm St. Dev. \\
\hline 1 & $0.250 \pm 0.000$ & $0.250 \pm 0.000$ & $0.250 \pm 0.000$ & $0.250 \pm 0.000$ & $0.769 \pm 0.137$ \\
\hline 2 & $0.250 \pm 0.000$ & $0.650 \pm 0.347$ & $0.852 \pm 0.027$ & $0.880 \pm 0.044$ & $0.865 \pm 0.039$ \\
\hline 3 & $0.250 \pm 0.000$ & $0.480 \pm 0.399$ & $0.924 \pm 0.010$ & $0.913 \pm 0.043$ & $0.922 \pm 0.044$ \\
\hline 5 & $0.709 \pm 0.398$ & $0.946 \pm 0.019$ & $0.947 \pm 0.007$ & $0.942 \pm 0.014$ & $0.949 \pm 0.007$ \\
\hline 10 & $0.953 \pm 0.005$ & $0.958 \pm 0.007$ & $0.961 \pm 0.011$ & $0.957 \pm 0.002$ & $0.956 \pm 0.005$ \\
\hline 20 & $0.966 \pm 0.008$ & $0.964 \pm 0.005$ & $0.968 \pm 0.002$ & $0.965 \pm 0.003$ & $0.962 \pm 0.000$ \\
\hline 30 & $0.963 \pm 0.005$ & $0.964 \pm 0.002$ & $0.966 \pm 0.002$ & $0.965 \pm 0.000$ & $0.964 \pm 0.002$ \\
\hline 40 & $0.962 \pm 0.007$ & $0.962 \pm 0.006$ & $0.957 \pm 0.005$ & $0.959 \pm 0.012$ & $0.964 \pm 0.005$ \\
\hline 50 & $0.957 \pm 0.005$ & $0.953 \pm 0.005$ & $0.947 \pm 0.012$ & $0.950 \pm 0.009$ & $0.954 \pm 0.002$ \\
\hline 100 & $0.949 \pm 0.005$ & $0.942 \pm 0.002$ & $0.949 \pm 0.009$ & $0.950 \pm 0.014$ & $0.951 \pm 0.006$ \\
\hline 200 & $0.934 \pm 0.024$ & $0.947 \pm 0.005$ & $0.950 \pm 0.009$ & $0.950 \pm 0.011$ & $0.955 \pm 0.006$ \\
\hline 300 & $0.943 \pm 0.002$ & $0.948 \pm 0.009$ & $0.949 \pm 0.002$ & $0.941 \pm 0.015$ & $0.942 \pm 0.009$ \\
\hline
\end{tabular}

Figure 8: Table with the mean accuracy \pm the standard deviation from the splice junction gene sequences with ESN results. 


\begin{tabular}{|c|c|c|c|c|c|}
\hline Spectral Radius: & 0.3 & 0.5 & 0.9 & 0.95 & 0.99 \\
\hline Nodes & Mean \pm St. Dev. & Mean \pm St. Dev. & Mean \pm St. Dev. & Mean \pm St. Dev. & Mean \pm St. Dev. \\
\hline 6 & $0.956 \pm 0.007$ & $0.960 \pm 0.013$ & $0.955 \pm 0.017$ & $0.955 \pm 0.015$ & $0.955 \pm 0.016$ \\
\hline 10 & $0.960 \pm 0.002$ & $0.962 \pm 0.003$ & $0.961 \pm 0.005$ & $0.962 \pm 0.006$ & $0.962 \pm 0.005$ \\
\hline 14 & $0.959 \pm 0.003$ & $0.959 \pm 0.002$ & $0.958 \pm 0.000$ & $0.957 \pm 0.001$ & $0.955 \pm 0.002$ \\
\hline 18 & $0.957 \pm 0.003$ & $0.958 \pm 0.002$ & $0.955 \pm 0.004$ & $0.955 \pm 0.002$ & $0.953 \pm 0.002$ \\
\hline 30 & $0.958 \pm 0.001$ & $0.956 \pm 0.002$ & $0.958 \pm 0.007$ & $0.958 \pm 0.007$ & $0.959 \pm 0.005$ \\
\hline 42 & $0.953 \pm 0.002$ & $0.954 \pm 0.005$ & $0.954 \pm 0.001$ & $0.955 \pm 0.002$ & $0.956 \pm 0.004$ \\
\hline 102 & $0.947 \pm 0.002$ & $0.948 \pm 0.002$ & $0.952 \pm 0.004$ & $0.952 \pm 0.003$ & $0.951 \pm 0.004$ \\
\hline 162 & $0.952 \pm 0.004$ & $0.950 \pm 0.002$ & $0.952 \pm 0.002$ & $0.951 \pm 0.002$ & $0.950 \pm 0.002$ \\
\hline
\end{tabular}

Figure 9: Table with the mean accuracy \pm the standard deviation from the splice junction gene sequences with the oscillating neural network results.

\begin{tabular}{|c|c|c|c|c|c|}
\hline Sparsity: & 0.1 & 0.25 & 0.5 & 0.75 & 1 \\
\hline Nodes & Mean \pm St. Dev. & Mean \pm St. Dev. & Mean \pm St. Dev. & Mean \pm St. Dev. & Mean \pm St. Dev. \\
\hline 1 & $0.469 \pm 0.074$ & $0.467 \pm 0.072$ & $0.479 \pm 0.084$ & $0.470 \pm 0.075$ & $0.481 \pm 0.084$ \\
\hline 2 & $0.522 \pm 0.055$ & $0.499 \pm 0.042$ & $0.511 \pm 0.048$ & $0.515 \pm 0.062$ & $0.517 \pm 0.071$ \\
\hline 3 & $0.539 \pm 0.091$ & $0.533 \pm 0.082$ & $0.558 \pm 0.049$ & $0.541 \pm 0.099$ & $0.551 \pm 0.056$ \\
\hline 5 & $0.668 \pm 0.039$ & $0.708 \pm 0.012$ & $0.652 \pm 0.033$ & $0.661 \pm 0.037$ & $0.674 \pm 0.053$ \\
\hline 10 & $0.723 \pm 0.003$ & $0.739 \pm 0.040$ & $0.737 \pm 0.024$ & $0.766 \pm 0.020$ & $0.757 \pm 0.024$ \\
\hline 20 & $0.840 \pm 0.052$ & $0.797 \pm 0.018$ & $0.781 \pm 0.034$ & $0.820 \pm 0.039$ & $0.815 \pm 0.067$ \\
\hline 30 & $0.792 \pm 0.049$ & $0.851 \pm 0.040$ & $0.844 \pm 0.035$ & $0.847 \pm 0.069$ & $0.864 \pm 0.037$ \\
\hline 50 & $0.935 \pm 0.008$ & $0.865 \pm 0.026$ & $0.917 \pm 0.025$ & $0.897 \pm 0.028$ & $0.897 \pm 0.031$ \\
\hline 100 & $0.957 \pm 0.012$ & $0.959 \pm 0.021$ & $0.964 \pm 0.007$ & $0.957 \pm 0.021$ & $0.962 \pm 0.016$ \\
\hline 200 & $0.941 \pm 0.040$ & $0.943 \pm 0.038$ & $0.841 \pm 0.209$ & $0.850 \pm 0.130$ & $0.938 \pm 0.041$ \\
\hline 300 & $0.823 \pm 0.112$ & $0.762 \pm 0.111$ & $0.752 \pm 0.096$ & $0.911 \pm 0.034$ & $0.825 \pm 0.110$ \\
\hline
\end{tabular}

Figure 10: Table with the mean accuracy \pm the standard deviation from the dengue virus genome with ESN results.

\begin{tabular}{|c|c|c|c|c|c|}
\hline Spectral Radius: & 0.3 & 0.5 & 0.9 & 0.95 & 0.99 \\
\hline Nodes & Mean \pm St. Dev. & Mean \pm St. Dev. & Mean \pm St. Dev. & Mean \pm St. Dev. & Mean \pm St. Dev. \\
\hline 6 & $0.678 \pm 0.019$ & $0.682 \pm 0.017$ & $0.703 \pm 0.015$ & $0.708 \pm 0.015$ & $0.712 \pm 0.016$ \\
\hline 10 & $0.703 \pm 0.004$ & $0.715 \pm 0.006$ & $0.774 \pm 0.021$ & $0.786 \pm 0.021$ & $0.787 \pm 0.023$ \\
\hline 14 & $0.708 \pm 0.009$ & $0.724 \pm 0.006$ & $0.795 \pm 0.036$ & $0.810 \pm 0.042$ & $0.818 \pm 0.045$ \\
\hline 18 & $0.715 \pm 0.003$ & $0.724 \pm 0.011$ & $0.838 \pm 0.013$ & $0.837 \pm 0.031$ & $0.852 \pm 0.035$ \\
\hline 30 & $0.718 \pm 0.006$ & $0.749 \pm 0.020$ & $0.875 \pm 0.024$ & $0.887 \pm 0.033$ & $0.885 \pm 0.059$ \\
\hline 42 & $0.713 \pm 0.001$ & $0.744 \pm 0.015$ & $0.890 \pm 0.011$ & $0.924 \pm 0.016$ & $0.926 \pm 0.024$ \\
\hline 102 & $0.724 \pm 0.013$ & $0.804 \pm 0.013$ & $0.961 \pm 0.019$ & $0.970 \pm 0.021$ & $0.982 \pm 0.002$ \\
\hline 162 & $0.716 \pm 0.023$ & $0.817 \pm 0.028$ & $0.765 \pm 0.112$ & $0.944 \pm 0.033$ & $0.952 \pm 0.044$ \\
\hline
\end{tabular}

Figure 11: Table with the mean accuracy \pm the standard deviation from the dengue virus genome with the oscillating neural network results. 